\title{
Implications of NEDD9 on AURKA activity: a biomarker for sensitization and therapeutic target in solid tumors
}

Ryan Joseph Ice

Follow this and additional works at: https://researchrepository.wvu.edu/etd

\section{Recommended Citation}

Ice, Ryan Joseph, "Implications of NEDD9 on AURKA activity: a biomarker for sensitization and therapeutic target in solid tumors" (2014). Graduate Theses, Dissertations, and Problem Reports. 7315. https://researchrepository.wvu.edu/etd/7315

This Dissertation is protected by copyright and/or related rights. It has been brought to you by the The Research Repository @ WVU with permission from the rights-holder(s). You are free to use this Dissertation in any way that is permitted by the copyright and related rights legislation that applies to your use. For other uses you must obtain permission from the rights-holder(s) directly, unless additional rights are indicated by a Creative Commons license in the record and/ or on the work itself. This Dissertation has been accepted for inclusion in WVU Graduate Theses, Dissertations, and Problem Reports collection by an authorized administrator of The Research Repository @ WVU.

For more information, please contact researchrepository@mail.wvu.edu. 


\title{
Implications of NEDD9 on AURKA activity: a biomarker for sensitization and therapeutic target in solid tumors.
}

\author{
Ryan Joseph Ice
}

\begin{abstract}
Dissertation Submitted to the School of Medicine at West Virginia University In Partial Fulfillment of the Requirements for the Degree of
\end{abstract}

Doctor of Philosophy in Cancer Cell Biology

\author{
Scott Weed, Ph.D., Chair \\ Michael Ruppert, M.D, Ph.D. \\ William Petros, Pharm.D. \\ Stephen Graber, Ph.D. \\ Alexey Ivanov, Ph.D. \\ Elena Pugacheva, Ph.D., Mentor \\ Cancer Cell Biology Program \\ Morgantown, West Virginia \\ 2013
}

Keywords: NEDD9, AURKA, ubiquitination, breast cancer 
UMI Number: 3618105

All rights reserved

INFORMATION TO ALL USERS

The quality of this reproduction is dependent upon the quality of the copy submitted.

In the unlikely event that the author did not send a complete manuscript and there are missing pages, these will be noted. Also, if material had to be removed, a note will indicate the deletion.

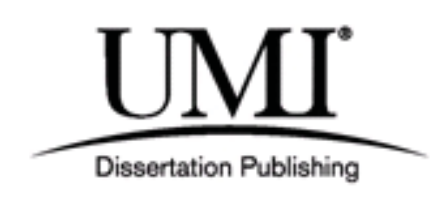

UMI 3618105

Published by ProQuest LLC (2014). Copyright in the Dissertation held by the Author.

Microform Edition (C) ProQuest LLC.

All rights reserved. This work is protected against unauthorized copying under Title 17, United States Code

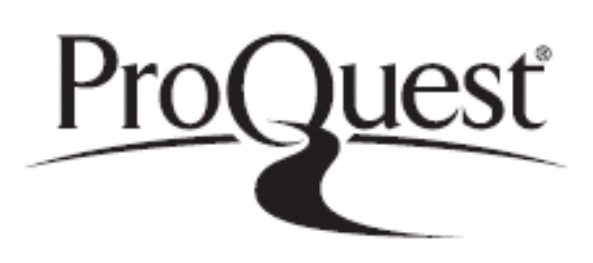

ProQuest LLC.

789 East Eisenhower Parkway

P.O. Box 1346

Ann Arbor, MI 48106 - 1346 


\begin{abstract}
Implications of NEDD9 on AURKA activity: a biomarker for sensitization and therapeutic target in solid tumors.
\end{abstract}

Ryan Joseph Ice

The spread of cancer, known as metastasis, is the major cause of relapse and inability to cure disease. Invasion of cancer cells into neighboring tissue and to distant sites allows cancer cells to evade therapeutic agents, due to the low therapeutic penetration of certain tissues such as the central nervous system, and eliminates the feasibility of resection; which is why the study of pro-metastatic proteins is of great urgency, one such protein is NEDD9. NEDD9 is overexpressed in various tumor types and is correlated with poor prognosis due to a more invasive and metastatic phenotype. NEDD9 serves as a scaffolding protein at the intersection between cell proliferation, through activation of mitotic Aurora A kinase (AURKA), and invasion, through regulation of Src kinase and matrix metalloproteinase 14, MMP14, activity. The overall aim of my work is to determine the role of NEDD9 in tumorigenesis and its implications on AURKA activity in tumor cells. AURKA is up regulated in the majority of human cancers, but not in normal cells making it a promising target for therapeutic interventions. There are several highly specific AURKA small molecule inhibitors in clinical trials to treat cancer. Nevertheless, it was unknown how NEDD9 binding to AURKA affects inhibitor efficacy, AURKA degradation, proliferation, and migration of tumor cells. My current studies have answered some of these critical questions allowing for further improvements of AURKA based therapeutics leading to prolonging patient survival. Study one demonstrates that NEDD9 protects AURKA from degradation and limits the successful application of AURKA inhibitors leading to greater metastasis. Additionally, we established that the AURKA inhibitor MLN8237 (Alisertib), currently in Phase III clinical trials to treat advanced lymphomas, has the ability to eliminate breast cancer metastases. Study two establishes NEDD9 as a necessary scaffolding protein for invasion of breast cancer cells by regulating the activity of a key extracellular matrix (ECM) degrading enzymes such as MMP14 and its inhibitor, TIMP2. Study three illustrates the ability of NEDD9 overexpression to increase MMP dependent mammary gland development and potentially promote tumorigenesis in a transgenic animal model. Furthermore, study three details the development of a conditional NEDD9 transgenic mouse strain that can be utilized for various studies. Finally, study four details the method and feasibility of utilizing patient derived xenograft models for future AURKA inhibitor testing in the treatment and prevention of metastatic breast cancer. Taken together, this thesis illustrates the importance and clinical applications of studying the NEDD9-AURKA signaling hub in treating tumor metastasis. 


\section{Acknowledgements}

I would like to thank my advisor Dr. Elena Pugacheva, for teaching me how to become a productive scientist, for always supporting me through good and bad data days, and for being a demanding, yet patient mentor.

I would also like to acknowledge my Graduate Committee for their guidance, particularly my Graduate Committee Chair and Graduate Program Coordinator, Dr. Scott Weed for giving me direction and for always having an open door to students.

I would like to acknowledge all current and past lab members of the Pugacheva Lab for their objective opinion and critics.

I would like to thank Dr. Scott Hiebert at Vanderbilt University for allowing me to take classes while I worked for him as a research assistant, and for encouraging me to go to graduate school.

I would also like to thank my family, especially my parents, Susan and Ralph Ice, for indulging my curiosity as a child and encouraging me throughout my studies.

Finally, last but certainly not the least, I would like to thank my wife, Varvara Kozyreva, for putting up with my workaholic nature and for being so understanding and supportive. 


\section{Table of Contents}

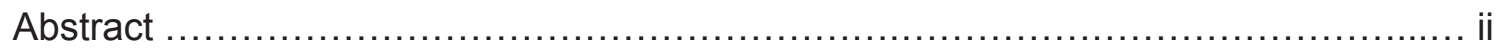

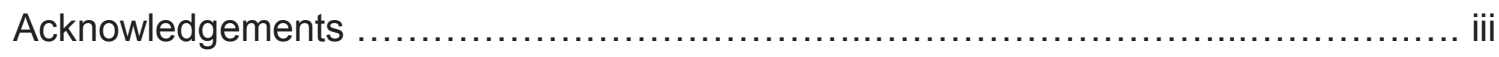

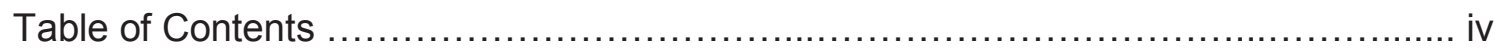

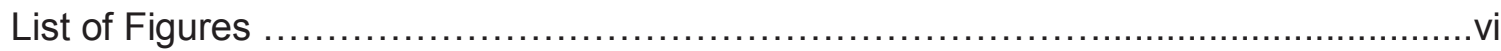

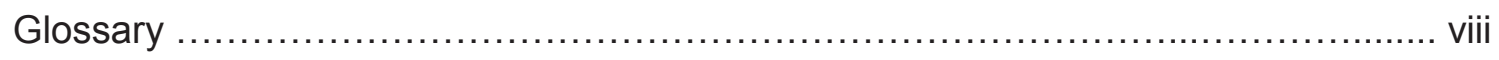

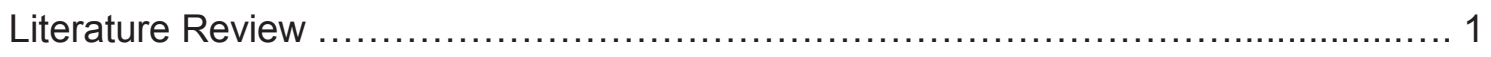

1. AURKA: Cell Cycle Regulator and Oncogene

1.1 AURKA activation and domain structure

1.2 The role of AURKA in the regulation of cell cycle and aneuploidy

1.3 The function of AURKA in tumorigenesis

1.4 Expression of AURKA in human cancers

1.5 The impact of AURKA expression in drug resistance and cancer treatment

1.6 Animal models of AURKA over-expression and cancer

1.7 The role of AURKA in invasion and metastasis

2. AURKA Inhibitors: A promising therapeutic target

2.1 Rational and Development of AURKA inhibitors

2.2 The efficacy of AURKA inhibitors in vitro and clinical trials

2.3 The impact of AURKA binding partners on the efficacy of AURKA Inhibitors

3. NEDD9: The oncogenic scaffolding protein

3.1 Structure, binding partners, and post-translatable Modifications of NEDD9

3.2 NEDD9 expression in Human Cancers

3.3 The role of NEDD9 in Invasion \& Migration

3.4 The role of NEDD9 in regulation of the Cell Cycle and AURKA

3.5 Transcript regulation of NEDD9 expression in normal and tumor cells

4. Regulation of NEDD9 and AURKA through degradation 
5. Mouse transgenic models in human breast cancer research

6. Patient derived xenografts $(\mathrm{pdx})$ as promising new model for preclinical anti-cancer drug testing

Study 1: NEDD9 Depletion Destabilizes Aurora A Kinase and Heightens the Efficacy of Aurora A Inhibitors: Implications for Treatment of Metastatic Solid Tumors 26

Study 2: Depletion of NEDD9 leads to inactivation of MMP14 by TIMP2 and prevents invasion and metastasis of breast cancer cells 60

Study 3: Generation of a Mammary Specific NEDD9 Knock-in Mouse Model and analysis of NEDD9 overexpression impact of mammary gland development 92

Study 4: Development of breast cancer patient derived xenograft (BCa-PDX) models to test for sensitivity to AURKA inhibitor/s and to identify the new biomarkers indicative of treatment outcome 106

General Discussion 


\section{List of Figures}

\section{Literature Review}

1. Diagram of AURKA domain structure

2. Diagram of NEDD9 domains

\section{Study 1}

1. Increased NEDD9 expression correlates with expression of AURKA protein in invasive ductal breast adenocarcinomas.

2. Depletion of NEDD9 leads to dramatic decrease of AURKA protein. Western blot analysis of NEDD9 and AURKA in breast cancer cell lines

3. NEDD9 regulates stability of AURKA through inhibition of proteasome-dependent degradation.

4. NEDD9 binding to AURKA is necessary for protein stabilization.

5. NEDD9 binding to AURKA decreases the efficacy of AURKA inhibitors in vitro.

6. Depletion of NEDD9 combined with AURKA inhibitors leads to decrease in a tumor burden and lung metastasis in breast cancer xenograft model.

7. Combination of AURKA Inhibitors with depletion of NEDD9 reduces colonization potential of human circulating tumor cells in lungs.

\section{Supplemental Figures:}

1. Depletion of NEDD9 with siRNA does not affect transcription or cell cycle distribution, but decreases AURKA protein levels.

2. Combination of AURKA inhibitors with Depletion of NEDD9 dramatically reduces metastasis to lungs.

\section{Study 2}

1. NEDD9 is overexpressed in invasive breast ductal carcinomas and BCa cell lines.

2. NEDD9 localizes to and promotes invadopodia maturation.

3. NEDD9 depletion ablates matrix degradation and invasion in 3D matrices.

4. NEDD9 depletion decreases MMPs activity, but does not affect MMPs expression or secretion. 
5. NEDD9 depletion leads to increase in amount of surface-bound TIMP2 and MMP14:

6. Inhibition or depletion of MMP14 or TIMP2 recapitulates invasion deficiency of shNEDD9 cells.

7. NEDD9 depletion inhibits metastasis in xenograft models.

Supplementary Figures:

1. Supplemental Figure 1

2. Supplemental Figure 2

3. Supplemental Figure 3

\section{Study 3}

1. Design and expression of Cre inducible nedd9 knock-in.

2. Genotyping and testing of Cre-NEDD9 mouse model.

3. NEDD9 overexpression results in increased budding of mammary glands.

\section{Study 4}

1. Successful engraftment of patient derived xenograft tumor. 


\section{Glossary}

AURKA- Aurora Kinase A

AURKB- Aurora Kinase B

AURKC- Aurora Kinase C

NEDD9- neural precursor cell expressed, developmentally down-regulated 9

MMP- matrix metalloproteinase

BCa- breast cancer

Ki- inhibition constant

PDX- patient derived xenograft

MMTV- mouse mammary tumor virus

TMA- tissue microarray

WT- wild type

$\mathrm{KI}$ - knock in

KO- knock out

PyVmT- polyoma virus middle T antigen

Her2/Erb2- human epidermal growth factor receptor 2

TIMP2- tissue inhibitor of metalloproteinases 2

shRNA- small hairpin ribonucleic acid

PP2A- protein phosphatase 2A

aa- amino acid

MTOC- microtubule organizing center

TPX2- targeting protein for xenopus KIp2

Mdm2- mouse double minute 2 homolog

BRCA2- breast cancer anti-estrogen resistance 2

RalA- Ras related protein $A$

Akt- protein kinase B

ATP-adenosine triphosphoate

$A^{0}$ - angstrom

IC50- half maximal inhibitory concentration

Ras- rat sarcoma

IL6- interleukin 6

Cas- CRK-associated substrate 
FAK- focal adhesion kinase

Cbl- Casitas B-lineage Lymphoma

DOCK- docking protein

CrkL- v-crk sarcoma virus CT10 oncogene homolog (avian)-like

Abl- Abelson

Rap1- Repressor/activator site-binding protein

JNK- C-Jun kinase

TGFb- Transforming growth factor beta

HIF1a- Hypoxia-inducible factor 1-alpha

BCR- B cell receptor

TCR- T cell receptor

EGF- epithelial growth factor

HRG- heregulin

EMT- epithelial to mesenchymal transition

VHL- von Hippel-Lindau

PDGF- platelet-derived growth factor receptor

WAVE- Wiskott-Aldrich syndrome protein family member

GAP- GTPase-activating proteins

GEF- guanine nucleotide exchange factor

VEGF- Vascular endothelial growth factor

Ect2- Epithelial cell-transforming sequence 2

HDAC6- histone deacetylase 6

Cox-2- Cytochrome c oxidase subunit 2

FoxC1- forkhead box C1

mTOR- Mammalian target of rapamycin

cdh1- CDC20 homolog 1

APC/C- anaphase promoting complex

Smad- mothers against decapentaplegic homolog

WAP- whey acidic protein

BLG- beta-Lactoglobulin 


\section{Literature Review}

\section{AURKA: Cell Cycle Regulator and Oncogene}

\subsection{AURKA activation and domain structure}

Aurora Kinase A, also known as AURKA, is a mitotic serine/threonine kinase activated by an autophosphorylation at amino acid, threonine 288 , near the activation loop of the catalytic domain. Upon phosphorylating threonine 288, the activity of AURKA dramatically increases (Walter 2000, Ohashi 2006). The AURKA inactivation is regulated by protein phosphatase type 2a (PP2a) which dephosphorylates and inactivates AURKA (Katayama 2001). Additionally, AURKA activity is curtailed through proteasome mediated degradation, which will be covered in later chapters. AURKA belongs to the Aurora family of proteins, consisting of three known members: Aurora A, Aurora B and C. All Aurora kinases possess a variable amino terminal domain (N) along with a conserved catalytic domain (Cat) (Fig.1).

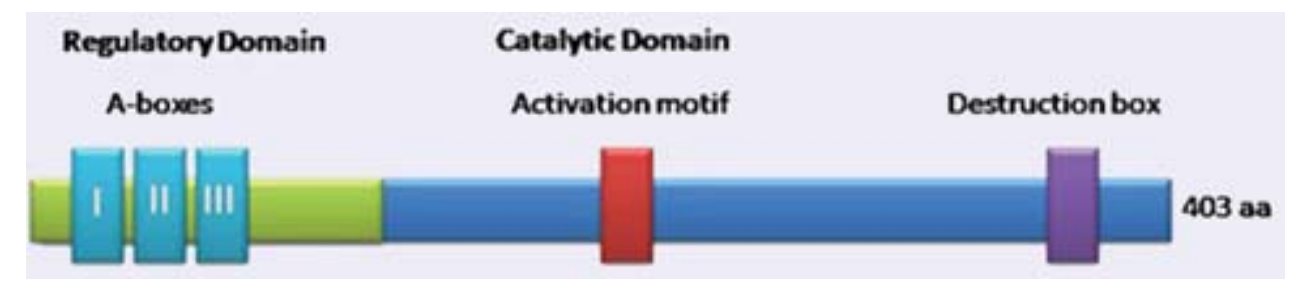

Fig. 1 Structure of AURKA. Illustrating the regulatory domain and catalytic domain (http://atlasgeneticsoncology.org)

Despite the structural similarity, the role of AURKA is distinct from Aurora B and C. The Nterminal domain (aa 1-128) allows AURKA to localize to centrosomes (MTOC-microtubule organizing centers) and interact with microtubules during mitosis (Giet 2001). The structure of AURKA is such that the N-terminal domain inhibits the catalytic domain when AURKA is not bound to AURKA activating proteins (Zhang 2007). Binding partners and activators, such as NEDD9 and TPX2, allow for the release of autoinhibition, resulting in autophosphorylation of threonine 288 (Pugacheva 2007, Kufer 2002). Once AURKA is activated, phosphorylation of serine 51, known as the A-Box, is achieved via autophosphorylation protecting AURKA from degradation (Littlepage 2002). Finally, active AURKA is targeted then to the centrosome through microtubule binding were it can function as a mitotic kinase.

1.2 The role of AURKA in the regulation of cell cycle and aneuploidy 
Entry into mitosis, one of the phases of cell cycle, is controlled through the activation of Cdk1 by forming a complex with CyclinB1 (Gavet 2010). AURKA regulates the formation of this complex through phosphorylation and activation of cdc25b phosphatase, which removes an inhibitory phosphorylation from Cdk1 (Horn 2007). Accumulation of AURKA at the centrosome is required for centrosome maturation and segregation (Wang 2010). After initiation of mitosis and nuclear envelop break down AURKA is targeted from the centrosome to mitotic spindle through its association with microtubule binding protein TPX2 (Kufer 2002). Microtubule bound AURKA continues to phosphorylate multiple targets leading to their loading on to the mitotic spindle thus orchestrating spindle assembly. By regulating both CyclinB1/Cdk1 complex activity and mitotic spindle loading, AURKA regulates both early G2/M phase transition and mitosis itself. Absence of AURKA in normal cells results in G2/M arrest or if cells already passed G2/M, the formation of a monopolar spindle, inability to separate DNA and lethality (Glover 1995). Similar phenotypes are also present in knockout embryos of AURKA knockout mice being non-viable even at day 3.5 due to formation of blastocyst consisting of multinucleated cells leading to chromosome disarray, and retarded growth (Sasai 2008).

\subsection{The function of AURKA in tumorigenesis}

Overexpression of AURKA, which is common in most cancers, leads to amplification of centrosomes and multipolar spindle formation resulting in aneuploidy, due to the miss segregation of chromosomes during mitosis (Tatsuka, 1998). Under normal circumstances aneuploidy will lead to apoptosis, controlled cell death; however, in the absence of necessary cell cycle checkpoints, such as p53, the cancer cell will continue to divide resulting in chromosome instability. Chromosome instability, a hallmark of many cancers, leads to an increase in mutation rate and a more aggressive phenotype (Meraldi, 2002). To exacerbate the phenotype, AURKA has been shown to phosphorylate p53 at serine 215 , which inhibits p53 DNA binding and transactivation activity (Liu 2004). Other studies show that AURKA is able to phosphorylate p53 at serine 315 which targets p53 for degradation by Mdm2 via proteolysis (Katayama 2004). Overexpression of AURKA will result in a decrease in p53, promoting chromosome instability. Since p53 is a major cell cycle and DNA damage checkpoint the result of p53 inhibition is increased resistance to radiotherapy and chemotherapeutic drugs, such as cisplatin and gamma-radiation, which rely on DNA damage checkpoints to induce apoptosis.

\subsection{Expression of AURKA in human cancers}

AURKA was first discovered to be a part of an amplified portion of chromosome 20q13 during a colon cancer screen. The levels of both AURKA protein and mRNA are increased in 
more than $50 \%$ of primary colon cancers and are increased in breast and other solid tumors (Bischoff 1998). Independently, AURKA is seen to be amplified in $12 \%$ of primary human breast tumors (Zhou, 1998). AURKA is an oncogene and when overexpressed AURKA is able to transform rodent fibroblasts and mouse 3T3 cells (Bischoff 1998, Zhou 1998) stating the importance of AURKA regulation for normal cell growth. While gene amplification is one mechanism for increased AURKA mRNA and protein found in some breast, colon, prostate, neuroblastoma, and cervical cancer tumors increased levels of mRNA are also found independent of amplification suggesting that there are multiple pathways leading to increased levels of AURKA (Li 2003, Gritsko 2003, Reichardt 2003). In a survey of pancreatic cancers cell lines all nine tested and 22 of 38 pancreatic cancer patient tissues had from twice to ten times the amount of AURKA protein when compared to matching normal tissues (Li 2003). In fact when tested via immunohistochemical staining, elevated levels of AURKA protein are found in 94\% of invasive ductal breast adenocarcinomas; while AURKA is barely detectable in benign breast lesions (Tanaka 1999).

\subsection{The impact of AURKA expression in drug resistance and cancer treatment}

An important point for breast cancer treatment is the presence of estrogen and progesterone receptors in the tumor, as these receptors can be utilized as sites for targeted therapy. When breast cancer tumors are assessed for AURKA levels they positively correlated with both advanced stage and loss of estrogen and progesterone receptors. In other words, the more aggressive and harder to treat tumors had more AURKA present, thus indicating AURKA as a target for inhibitor therapy in tumors resistant to endocrine therapy (Miyoshi 2001). In other studies, high AURKA levels are detected in premalignant state tumors which were later resistant to hormone therapy in a carcinogen induced rat tumor model, suggesting once again that AURKA is a predictive marker for endocrine resistant phenotypes (Goepfert 2002).

The ability to create resistance to standard therapy is a hallmark of AURKA. High levels of AURKA not only allow tumor cells to accrue chromosomal abnormalities thereby increasing mutation rates, but also allow tumor cells to bypass key mitotic spindle checkpoints, thus making the cells resistant to the chemotherapeutic drugs such as paclitaxel (Anand 2003). However, the ubiquitousness of AURKA overexpression does allow for combinational therapies to be attempted and give hope for patients by utilizing AURKA inhibitors. Additionally, the ability of AURKA to repress the DNA damage checkpoint ability of p53 makes AURKA an important protein for combinational therapies, due to many chemotherapeutics needing p53 activity to induce apoptosis. More specific to breast cancer is the finding that familial breast cancers 
containing BRCA2 mutations have increased levels of AURKA, pointing to a site for targeted therapy for this very aggressive form of breast cancer (Bodvarsdottir 2007).

\subsection{Animal models of AURKA over-expression and cancer}

Proving AURKA to be an oncogene, AURKA overexpression is able to induce tumor formation by 20 months in genetic animal models where AURKA expression is driven by mouse mammary tumor virus (MMTV) promoter. Even before tumors formed, cells present with elevated Akt levels, the target of rapamycin, and high nucleated cyclin D1 levels resulting in increased rates of proliferation. Not surprisingly, the cells also presented with extreme chromosome abnormalities, and yet did not undergo apoptosis (Wang 2006). Additionally in the mouse mammary model system, when AURKA is overexpressed proliferation rates increase dramatically as does the levels of p53, due to chromosome defects. Surprisingly, the presence of high p53 does not induce apoptosis in these tumor cells. In the background of p53 inactivation, AURKA overexpression in the mammary fat pad is able to induce tumorigenesis at a faster rate, suggesting p53 pathway activation is overcome through selective adaption (Zhang 2004).

\subsection{The role of AURKA in invasion and metastasis}

While early detection and targeted therapies have increased the survivability of many cancers; once invasion from the primary site has occurred survivability of disease greatly decreases. Early reports show AURKA activity stimulates collagen I induced invasion, migration, and anchorage independent growth, via RalA phosphorylation. RalA is a member of the Ras family of proteins and is responsible for the formation of filopodia protrusions and vesicle trafficking (Wu 2005). AURKA has also been implicated in migration and invasion through its ability to phosphorylate Akt at serine 473, which leads to greater migration rates (Guan 2007). AURKA phosphorylates and activates the actin severing factor, cofilin, directly regulating actin dynamics involved in migration and invasion potential of cancer cells (Wang 2010); therefore, AURKA targeted therapies could prove efficient against invasive and metastatic cancers.

\section{AURKA Inhibitors: A promising therapeutic target}

\subsection{Rational and Development of AURKA inhibitors}

Kinases contain a conserved ATP binding site critical for enzymatic function and share a similar biochemical mechanism of action. They are considered as a first line for designing 
targeted therapies. By blocking the ATP binding site of a kinase, utilizing molecules that compete with ATP for binding, one can easily inhibit the enzymatic activity of the kinase. Out of the three Aurora family members, only AURKA and AURKB regulate the cell cycle and could be targeted to inhibit division; however, only AURKA expression and activity had been shown to correlate with survival of cancer patients. High AURKA protein levels are a negative indicator of survival (Nadler 2008). Inhibition of AURKA more efficiently induces mitotic deficiencies and apoptosis or senescence than inhibition of AURKB (Warner 2006). Additionally, since AURKA can repress the DNA damage checkpoint through inactivation of p53 inhibition of AURKA will be beneficial for many combinational therapies which rely on p53 activity to induce apoptosis.

The first attempt in designing effective AURKA inhibitors occurred once the crystal structure of AURKA was defined down to $1.9 \AA$ (Nowakowski, 2002). The next proof of concept experiments, utilizing computer modeling and inhibitor docking in silico followed by in vitro inhibitor kinase assays, proved AURKA to be a druggable target (Mahadevan 2003).

\subsection{The efficacy of AURKA inhibitors in vitro and clinical trials}

The development of VX-680, tozasertib, a pan Aurora inhibitor, with a Ki of AURKA 0.6nM, AURKB 18nM, and AURKC 4.6nM, demonstrated the first successful clinical application of Aurora inhibitors. VX-680 shows efficacy in xenograft experiments for leukemia, colon and pancreatic tumors (Harrington 2004). Next, it was found in primary human and mouse prostate cancer cells that VX-680 is able to induce apoptosis; additionally, VX-680 is able to increase apoptosis by two fold when combined with doxorubicin, a chemotherapeutic (Lee 2006).Another inhibitor of the Aurora family, ZM447439, showed the ability to inhibit chromosome condensation and microtubule spindle formation; however due to the higher efficacy in targeting AURKB $(\mathrm{Ki}=0.36 \mathrm{nM})$ than AURKA $(\mathrm{Ki}=100 \mathrm{nM})$ the inhibitor was unable to induce apoptosis while increasing aneuploidy (Gadea 2005). Showing promise as a drug target, further modeling and drug design was performed to target AURKA, introducing the use of tricyclic scaffolds (Warner 2006, Heron 2006). Building upon this work, a second generation of AURKA inhibitors was developed: PHA-680632, with an IC50 of 27nM for AURKA and 135nM for AURKB, shows promise by inducing growth arrest in various tumor line xenografts and a transgenic breast cancer line. However, the inhibitor was unable to eliminate disease by decreasing tumor burden (Soncini 2006).

By investigating the mechanism of growth arrest and apoptosis induced by the various inhibitors one can understand which protein to target, AURKA or AURKB, and what mitotic checkpoints are needed to induce apoptosis. Looking at multiple cell lines, both with and without 
p53 and p21, researchers were able to discover that VX-680 induces two types of apoptosis. In cells containing wild type p53 and p21 cells undergo apoptosis upon entering mitosis, due to DNA duplication accompanied by a lack of segregation of chromosomes. In cells lacking p53 and/or p21, cells undergo endoreduplication, genome duplication without cytokinesis, to the extent of being non-viable (Gizatullin 2006). The ability of AURKA inhibitor, PHA-680632, to induce apoptosis in p53 null cells is further shown in HCT116 colon cancer xenograft experiments, illustrating the additive effect of AURKA inhibitors when combined with radiotherapy. The additive effect of AURKA inhibitors is due in part to p53 deficient cells being selected for when DNA damage agents are utilized (Tao 2007). VX-680 also shows much greater cytotoxicity than ZM447439, an AURKB specific inhibitor, suggesting that induction of apoptosis is through AURKA (Tyler 2007). Studies also show that AURKA inhibition in multiple myeloma cells leads to apoptosis via aneuploidy, with neither IL-6 nor activated Ras pro-survival pathways protecting the cells from apoptosis (Shi 2007). Further investigation of AURKA inhibition induced apoptosis, via the AURKA specific inhibitor MLN8054, reveals that the mechanism of action is not necessarily decreased mitosis but deleterious aneuploidy (Hoar 2007). The next generation variant of MLN8054, MLN8237 (alisertib), is currently in Phase IV clinical trials for treatment of myeloid lymphoma and melanoma,

\subsection{The impact of AURKA binding partners on the efficacy of AURKA Inhibitors}

Since AURKA activation is induced through binding partner associations and autophosphorylation, it would stand to reason that AURKA interactors would influence the efficacy of inhibitions. Examples of AURKA activators are TPX2, AJUBA, and NEDD9 (Eyers 2003, Hirota 2003, Pugacheva 2007). TPX2, targeting protein for Xklp2, is a mitotic protein present at the start of the G1/S transition and is degraded at the end of cytokinesis. TPX2 binds the carboxyl terminus of AURKA in a phosphate-independent manner allowing for a local conformational change in the activation domain, resulting in both AURKA autophosphorylation and protection from phosphotases, therefore locking AURKA in its active state (Kufer 2002, Eyers 2003, Bayliss 2003). More explicitly, through binding TPX2, the glycine rich loop, proximal to the adenosine triphosphate binding site in the amino acid terminus of AURKA, swings via a hinge region to protect AURKA from dephosphorylation (Eyers 2005).

Utilizing the known dynamics of TPX2 activation of AURKA, computer modeling of AURKA, both with and without activator binding, establishes that the hydrophobic pocket adjacent to the adenosine triphosphate (ATP) binding site, which many inhibitors use to lodge in the active site, is closed upon TPX2 binding (Anderson 2007). Computer modeling experiments 
were confirmed by the co-crystal structure of bound AURKA/TPX2/VX-680 down to a resolution of $2.3 \AA$. The structure shows the binding of VX-680 to AURKA/TPX2 in a completely novel manner, via binding of the glycine rich hinge region in the hinge region AURKA (Zhao 2008). These findings support the claim that AURKA binding partners influence heavily AURKA inhibitor binding profile, pharmacodynamics, and are areas for further investigation.

Combination therapies utilizing AURKA inhibitors and Src inhibitors show efficacy and synergistic effects. Both Src and AURKA interact with NEDD9, serving as a point of crosstalk between the two pathways, pointing to the importance of AURKA interactors as a source for combination therapy design (Ratushny 2012).

\section{NEDD9: The oncogenic scaffolding protein}

\subsection{Structure, binding partners, and post-translatable modifications of NEDD9}

The Crk-Associated Substrate (Cas) family of proteins contains four members: BCAR1/p130Cas, EfS/Sin1, NEDD9/HEF1/Cas-L, and CASS4/HEPL. Cas proteins function as scaffolding proteins, serving as a platform for proteins to bind therefore increasing the likelihood of a reaction taking place. Additionally, the Cas family of proteins serves as a mediators of signaling through post-translational modifications, such as phosphorylation, leading to a change in protein binding affinities and conformational changes. Between NEDD9, Neural cell precursor expressed developmentally down-regulated 9, and p130Cas, p130Cas is ubiquitously expressed in all tissues and is found localized to focal adhesions within cells; while, NEDD9 is tissue specific and is found throughout the cell. Both proteins contain four domains: 1) a Src homology 3 domain (SH3) which is able to bind proline rich regions of neighboring proteins; 2) a substrate domain, containing multiple tyrosine residues, which upon phosphorylation serve a docking sites for other proteins; 3) a serine rich region, and 4) a carboxyl terminal domain which contains the focal adhesion targeting region (Fig.2).

\section{\begin{tabular}{l|l|l|l} 
SH3 & SD & SR & CT \\
\hline
\end{tabular}}

Figure 2. Diagram of NEDD9 domains

Both, NEDD9 and p130Cas are able to be phosphorylated by various kinases, such as Src, focal adhesion kinase (FAK), and Abl (Law 1996). To further complicate the parsing of roles 
between p130Cas and NEDD9, the two proteins heterodimerize via a Helix Loop Helix (HLH) motif in the carboxyl terminal domain of the two proteins (Law 1999).

One way to tease out the role of NEDD9 apart from p130Cas is to look at lymphocytes which naturally lack p130Cas. In T lymphocytes, NEDD9 is phosphorylated in response to activation of integrin $\beta 1$ on the cell surface via direct binding of FAK and NEDD9 (Minegishi 1996, Van Seventer 2001). In addition to activating T cells, NEDD9 plays a role in the B cell activation cascade. Upon ligands binding $B$ cell antigen receptors (BCR) on the surface of $B$ cells, NEDD9 is phosphorylated as a part of a signaling cascade ending in the rearrangement of the actin cytoskeleton promoting division and migration (Manié 1997). NEDD9 also plays a role in myeloid cell activation by serving as a critical hub for phosphorylation and binding. Upon myeloid immunoreceptor tyrosine-based activation motif (ITAM) binding on the cell surface, Cbl, Crkl, and NEDD9 are tyrosine phosphorylated forming a complex leading to Rap1 activation, a small GTPase, resulting in the loosening of cell adherence junctions (Kyono 1998).

Functioning as a critical scaffolding protein allows NEDD9 to influence a multitude of signaling pathways. As a result of FAK phosphorylation, in the presence of integrin $\beta 1$, phosphorylated NEDD9 levels, increasing activation of such pathways as: Jun N-terminal protein kinase (JNK), transforming growth factor-beta (TGF $\beta$ ), hypoxia-inducible factors 1 \& 2 (HIF), and suppression E-cadherin junctions. In the case of the JNK pathway, phosphorylated NEDD9 binds to active JNK leading to a further increase in NEDD9 levels in a positive feedback loop, demonstrating the ability of NEDD9 to regulate JNK dependent apoptosis (Law 2000). NEDD9 influences the TGF $\beta$ pathway through sequestering Smad6 and Smad7, negative regulators of the TGF $\beta$ pathway (Inamoto 2007). Additionally, NEDD9 functions as a hypoxia inducible factor, since inactivation of von Hippel-Lindau (VHL) tumor suppressor activates HIF leading to phosphorylation of NEDD9 (Xu 2010). NEDD9 is also phosphorylated in response to cells being stimulated by epithelial growth factor (EGF) and heregulin (HRG) leading to MAPK activation (Nagashima 2008).

As with all phosphorylated events, a phosphatase is needed to negatively regulate the binding cascade; similar to the mechanism to inactivate AURKA, NEDD9 is dephosphorylation by PP2A thereby enabling the cell to reset the binding cascade of NEDD9 (Bradbury 2012).

\subsection{NEDD9 expression in Human Cancers}

To understand the importance of a protein in cancer one can look at how ubiquitously the protein is found to be up or down regulated in various cancer types. Increased levels of an 
individual protein can be caused through a multitude of mechanisms such as: increased transcription, decreased degradation, targeting by microRNA, and, in the case of cancer, increasing the number of copies in the genome.

NEDD9 is found to be upregulated in Philadelphia chromosome positive leukemia, where NEDD9 functions as a scaffold between oncogenic Bcr/Abl and downstream effectors Crkl and Cbl (de Jong 1997). By comparing multiple melanoma cell lines, NEDD9 is shown to be the only gene consistently amplified resulting in overexpression of the protein, leading to increased invasion and migration of melanocytoes (Kim 2006). Corroborating this finding, in patient samples $(n=19)$ the NEDD9 locus 6 p24 is seen by fluorescence in situ hybridization (FISH) to be amplified in $57 \%$ of melanoma patients (Moore 2008). Additionally, in matching normal and lung adenocarcinomas samples NEDD9 tumor expression and protein levels are significantly increased, correlating with tumor stage and grade, leading to poor prognosis (Chang 2012, Kondo 2012). Similar findings are seen in matching pancreatic ductal adenocarcinoma samples, increasing amounts of NEDD9 correlate with poor prognosis and higher grade tumors (Xue 2013). In glioblastoma patient samples, microRNA-145 (miR-145) is downregulated resulting in an increase in NEDD9 expression correlating to higher grade and decreased patient survival (Speranza 2012). Taking a closer look at lung adenocarcinomas NEDD9 correlated with hallmarks of epithelial to mesenchymal transition (EMT), such as fibronectin, and abnormal E-cadherin and $\beta$-catenin expression along with an increase in $\mathrm{N}$ cadherin (Miao 2013). The importance of NEDD9 in carcinogenesis was further demonstrated, in an oncogene-driven genetic mouse model of mammary tumors (MMTV-PyVmT). The NEDD9 knockout in this model led to a significant delay in cancer initiation, decrease in cancer growth, overall tumor burden, and metastasis. The cancer cells from tumors produced in these animals have dramatic decreases in FAK, Src, and Akt activity, along with deficiencies in invasion and migration (Izumchenko 2009).

\subsection{The role of NEDD9 in Invasion \& Migration}

NEDD9 functions in lymphoblastic cells as a scaffolding protein, allowing for crosstalk between signaling pathways in response to integrin $\beta 1$ and $T$ cell receptor (TCR) stimulation (Kamiguchi 1999), and leading to lymphocytic migration. Binding to extracellular matrixes and TCR stimuli results in phosphorylation of NEDD9, changing the binding characteristics of the protein, and thereby inducing migration and invasion (Ohashi 1999). NEDD9 is also important for the formation of neurite protrusions of neurons (Bargon 2005). In embryonic development, NEDD9 is essential for neuronal crest cell migration (Aquino 2009). Focal adhesion kinase 
(FAK) serves as the link between surface signaling and NEDD9 phosphorylation. When FAK binds activated integrins, FAK phosphorylates itself and NEDD9, inducing migration (Van Seventer 2001). NEDD9 is critical for migration of glioblastoma cells in response to platelet derived growth factor (PDGF) via NEDD9 localization to focal adhesions. NEDD9 plays such an important part of the focal adhesion kinase (FAK) cascade that the increase in invasion and migration due to overexpressed FAK is abrogated when NEDD9 expression is impeded (Natarajan 2006).

Cells have two classes of movement, mesenchymal and ameboid. Mesenchymal movement is characterized by well-developed adhesions and matrix degradation through the formation of ventral actin based protrusions known as invadopodia, allowing the cell to pierce through basal membranes and other heavily cross-linked extracellular matrixes. Ameboid movement is characterized by few adhesion points and a matrix contraction (without degradation) based movement via actin-myosin cytoskeleton dynamics through lightly or noncross-linked extracellular environment. Mesenchymal movement is driven by activation of the GTPase Rac, while ameboid movement is driven by the GTPase Rho. NEDD9 regulates the switch between the two types of movement by activating Rac and inhibiting Rho through downstream effectors (Sanz-Moreno 2008). NEDD9 forms a complex with Rac and DOCK6 thereby activating Rac. Activated Rac inhibits Rho via WAVE2. TGF $\beta$, known activator of EMT, induces mesenchymal movement via upregulation of NEDD9 (Giampieri 2009). Another mesenchymal promoting factor is vascular endothelial growth factor (VEGF). VEGF induces the formation of invadopodia in a NEDD9 dependent manner (Lucas 2010).

In addition to functioning directly as a switch between mesenchymal and ameboid movement, NEDD9 is a known epithelial to mesenchymal transition (EMT) inducer. NEDD9 has the ability, through Src, to disrupt the localization of E-cadherin to the cell surface and promote lysosomal degradation of E-cadherin (Tikhmyanova 2011, Kong 2011). E-cadherin is necessary for epithelial cell-cell junctions. In the absence of proper E-cadherin localization the cell can, transform into a more invasive mesenchymal-like phenotype resulting in potentially greater metastasis. Silencing of NEDD9 in low E-cadherin expressing cells results in reduced FAK and Src phosphorylation and restores epithelial morphology (Sima 2013). Other prominent EMT transducer proteins, such as Twist, also upregulate NEDD9 expression along with Rac1 and DOCK3 in order to promote mesenchymal type movement of the cell (Yang 2012).

3.4 The role of NEDD9 in regulation of the Cell Cycle and AURKA. 
While NEDD9 is present during all phases of the cell cycle it is upregulated significantly during the G2/M transition and early stages of mitosis. During mitosis NEDD9 localizes to the mitotic spindle poles (centrosomes) and mitotic spindle (Law 1998). At the centrosomes, NEDD9 binds to and changes the conformation of AURKA, allowing for AURKA to undergo autophosphorylation and activation. Overexpression of NEDD9 leads to an increase in centrosome number and multipolar spindles, resulting in an aneuploidy phenotype similar to AURKA overexpression (Pugacheva 2005). Interestingly, the amoeboid movement promoting protein, RhoA is necessary for cell rounding during mitosis and RhoA inactivation is necessary for cytokinesis, the division of cytoplasm. NEDD9 functions to activate RhoA, via the mitotic guanine exchange factor (GEF) Ect2; therefore, overexpression of NEDD9 could lead to cytokinetic defects and polyploidy (Dadke 2006).

NEDD9 also functions to regulate the cell cycle by regulating primary cilia. Primary cilia are found on the apical surface of epithelial cells. The cilium is comprised of microtubules with the lone centriole of the cell at the base, because of this there is only one cilium per cell. In preparation for entering the cell cycle, primary cilia must be disassembled. NEDD9, in conjunction with AURKA, sits at the base of the cilia and promotes microtubule deacetylation and depolymerization via HDAC6 phosphorylation leading to cilia disassembly and initiation of mitosis (Pugacheva 2007).

Cells lacking NEDD9 have a similar phenotype as cells with AURKA aberrations. While in murine spontaneous tumor models eliminating NEDD9 results in decreased invasion and later onset, the tumors that do develop have delayed mitosis and are hyperaggressive in part because of increased mutation rates due to mitotic defects. Increased mutation rates during tumorigenesis, similar to other paradigms such as antibiotic resistance, result in a delay in growth during the selection phase; however, once populations of cells have been selected the cells are more aggressive and resistant to treatment (Singh 2010).

\subsection{Transcript regulation of NEDD9 expression in normal and tumor cells}

Many signaling cascades involve increased transcription of NEDD9 leading to increases in invasion, migration, proliferation, and induction of EMT. The EMT promoting factor, TGF $\beta$ induces a sixteen fold increase in NEDD9 mRNA levels, resulting in an increase in Src and FAK activity (Zheng 2002). Increased levels of NEDD9 lead to an increase in TGF $\beta$ signaling through a positive feedback loop (Bruna 2012). Another EMT factor that can induce NEDD9 expression is FoxC1, a member of the Fox family of transcription factors. The ability of FoxC1 to induce migration and invasion is in part due to the upregulation of NEDD9 (Xia 2013). 
The established EMT inducing Wnt pathway is another cascade resulting in increases in NEDD9 transcription. Members of the canonical Wnt cascade such as: Wnt, $\beta$-catenin, and Dvl2 are all able to increase NEDD9 transcription; conversely, $\beta$-catenin knockdown results in decreased levels of NEDD9 (Li 2011). Upon treatment of cells with Wnt inhibitors NEDD9 levels drop, reducing invasion and migration of tumor cells independent of Src or FAK activation (lida 2012).

A second instance where NEDD9 expression is transcriptionaly induced is under hypoxic conditions. Cells experience hypoxia when the level of oxygen in the surrounding tissue does not meet the requirements of the cell. Tumors often experience hypoxia when growth outpaces angiogenesis and under inflammatory conditions. Hypoxia is known to induce EMT and increase migration and invasion of various tumors. Cyclooxygenase-2 (Cox-2) is activated under inflammatory conditions. Prostaglandin E(2), a downstream component of the Cox-2 pathway, induces NEDD9 expression thereby increasing cellular proliferation and migration (Xia 2010). Hypoxia inducible factor- $1 \alpha$ (HIF-1 $\alpha$ ) is a hypoxia induced transcription factor. NEDD9 is a direct transcriptional target of HIF- $1 \alpha$, leading to increased migration when HIF- $1 \alpha$ is induced. Upon NEDD9 depletion HIF-1 $\alpha$ is no longer able to induce migration, showing the critical nature of NEDD9 in this cascade. Interestingly, NEDD9 also enhances the transcriptional activity of HIF-1 $\alpha$, resulting in a positive feedback loop (Kim 2010). Yet another mechanism of induced NEDD9 expression in response to hypoxia is via the activation on ribosomal protein S6 kinase (S6K), which is a part of the mammalian target of rapamycin (mTOR) pathway. The mTOR/S6K pathway responds to metabolism disturbances, such as limited nutrients. Upon activation, S6K leads to transcriptional increases in hypoxia response genes, one of which is NEDD9 (Ismail 2012).

\section{Regulation of NEDD9 and AURKA through degradation}

It is important for the homeostasis of a cell to control the amount of each protein within it. Coordinated degradation of cell cycle regulated proteins is an example of how cells can accomplish the intricate balance of protein levels. It is necessary for mitotic proteins to be kept at low levels by degradation when the cell is not undergoing mitosis and to inhibit degradation during mitosis. In order to accomplish this task, the cell utilizes recognition subunits coupled to ubiquitin ligases. Each recognition subunit targets a specific set of proteins, thereby coordinating protein degradation depending on the cells requirements. Many proteins are degraded via the ubiquitin pathway, in which proteins are post translationally modified with ubiquitin molecules which signal to the cell that the proteins are to be degraded and trafficked to 
the proteasome. Proteins that are to be degraded are first bound by recognition subunits of ubiquitin ligases. Once bound by the full ubiquitin ligase complex, ubiquitin ligases along with recognition subunits, the protein is labeled with ubiquitin molecules and shuttled to the proteasome for degradation. By utilizing the same ubiquitin ligase and recognition subunits, cell cycle proteins are able to be degraded in a coordinated manner, signaling the end of mitosis.

In order to accomplish this coordinated destruction the cell employs the E3 ubiquitin ligase APC, anaphase promoting complex, and its recognition subunit cdh1. Cdh1 binds to cell cycle proteins at the end of mitosis and signals for APC to conjugate ubiquitin to the protein, thereby targeting the mitotic proteins to the proteasome for degradation. By degrading cell cycle proteins APC plays an important role in stopping proliferation. In a survey of 108 invasive breast cancers, APC was found to be decreased while mitotic index, histologic, and nuclear grade increased (Park 2005).

Both AURKA and NEDD9, the focus of this study, are degraded in a cdh1/APC dependent manner (Honda 2000). AURKA is targeted by cdh1 via the D-Box in the carboxy terminus and a unique A-Box in the variable amino terminus of the protein (Arlot-Bonnemains 2001, Taguchi 2002). The A-box functions as the main site of cdh1 binding, while the D-box functions as a second site after A-Box ubiquitination; therefore, allowing AURKA degradation to be independently regulated from AURKB, which contains only a D-box motif (Castro 2002). Multiple studies show that gene amplification does not account for the levels of accumulated AURKA in tumor cells; additional mechanism must be present to create such abundant AURKA detected in tissue and cell lines (Crane 2005, Klein 2005). Interestingly, phosphorylation of serine at amino acid position 51 in the A-box completely blocks cdh1 dependent degradation of AURKA, suggesting that dephosphorylation plays a role in both AURKA inactivation and degradation (Littlepage 2002, Kitajima 2007). In order to exit the cell cycle cdh1/APC ubiquitinates AURKA, leading to mitotic exit and cytokinesis. Knockdown of cdh1, via siRNA, leads to a similar phenotype as non-degradable AURKA mutants, failure of cytokinesis and aneuploidy (Floyd 2008).

Feedback loops also exist to keep NEDD9 levels in check during non-mitotic phases of the cell cycle. NEDD9 is targeted for destruction upon TGF $\beta$ induction, which was discussed earlier as a promoter of NEDD9 transcription, via Smad3 interacting with the APC/cdh1 complex. By both being able to induce transcription and increase NEDD9 degradation, TGF $\beta$ is able to keep NEDD9 transcription and protein increases transient (Li 2000, Nourry 2004). An additional mechanism to keep NEDD9 function in check is the ability of PP2A, a phosphatase 
that targets NEDD9, to protect NEDD9 from destruction. The preference to degrade phosphorylated NEDD9 illustrates how APC/cdh1 functions to keep NEDD9 activity at bay (Zheng 2006). Interestingly, the phosphorylation site that promotes APC/cdh1 binding is serine 396 not serine 296; indicating AURKA dependent phosphorylation is not a signal for NEDD9 destruction since serine 296 is the major target of AURKA phosphorylation (Hivert 2009).

\section{Genetic mouse models of human breast cancer}

While tissue culture systems are easy to use they are drastically oversimplified and artificial, due to the limited ability to reproduce the tumor microenvironment and the adaptation of the cells to grow on plastic. Genetic mouse models of human breast cancer provide a useful and informative alternative. There are multiple mammary specific promoters utilized in the field such as: mouse mammary tumor virus (MMTV), whey acidic protein (WAP), $\beta$ lactoglobin (BLG), and keratin 14 (K14) (Borowsky 2011). While the MMTV, WAP, and BLG promoters are expressed in luminal epithelial cells, the K14 promoter is expressed in the basal cells (Derksen 2006).

Utilizing these promoters one can overexpress an oncogene, a dominant negative tumor suppressor, or conditionally knockout a tumor suppressor to create mammary specific tumorigenesis. When tumor suppressors such as p53 are mutated heterogenic tumors develop with long latency due to accumulation of mutations. Simultaneous p53 and E-cadherin inactivation results in a phenotype very similar to invasive lobular carcinoma (Alvarez 2006).

The most characterized mouse model system is MMTV-PyVmt, polyoma virus middle T antigen. PyVmT is used to mimic the over expression of Erbb2, which is found to be overexpressed in 25\% human breast cancers (Lee 2010). PyVmT mimics the heterodimer Erbb2/Erbb3 in a dimerization and ligand-independent manner. Activation of Src, PP2A, and PI3K are found in both pathways (Dilworth 2002). Perhaps one of the major characteristics of this model system is that the tumors generated are very consistent. Tumorigenesis is $100 \%$ penetrant with tumors appearing at 20 weeks of age, with tumors initiated at 4 weeks of age. Mice often present from multiple foci tumors. Tumors also present with weak, though present, estrogen receptor signaling (Namba 2005).

Due to the early onset of tumorigenesis in the MMTV-PyVmT model, a good alternative is the MMTV-Her2 mouse model. Her2/Erbb2 heterodimerizes with other Her family members and activates the PI3K and MAP kinase pathways. Mice present with tumors between 8 to 12 months when the wild type Her2 is used and 5 to 10 months when the activated mutant form of the protein, neu, is used. Both neu and Her2 models present with frequent pulmonary 
metastasis (Muller 1998, Guy 1992). 37\% of tumors in the wild type Her2 overexpressing mice have missense mutations of the p53 gene, suggesting the p53 plays an important role in Her2 induced tumorigenesis (Li 1997).

\section{Patient derived xenografts (PDX) as a promising new model for preclinical anti- cancer drug testing}

Orthotopic human tumor xenografts contain distinct advantages over transgenic model systems since transgenic breast cancer in mice often does neither phenocopy human metastasis nor human breast cancer pathology. Through utilizing human tumor xenograft one can manipulate tissue culture cells in order to recapitulate genetic aberrations found in human tumor samples. Additionally, the inclusion of imaging reporters such as luciferase and RFP reduces the number of mice needed per study since euthanization is not necessary to measure tumor volume, burden, or metastasis (Jenkins 2005). Also, through the use of orthotopic human tumor xenografts, one is able to treat the same tumor with multiple treatments and different stages of disease.

Until recently, most existing in vivo orthotopic models utilized for preclinical assays of anticancer drugs were based on a limited number of cell lines; which were previously isolated from human tumors and selected through culturing before being implanted into immunodeficient animals. Unfortunately, cell lines do not reflect the heterogeneity of human breast cancer resulting in a weak correlation between animal study results and clinical results. In the Study 4, we developed new preclinical models based on engraftment of patient-derived tumor samples that were directly transplanted into animals. In contrast with cell line-derived xenografts, patienttumor xenografts maintain their cell differentiation, morphology, architecture, and the molecular signatures of the original patient tumors (DeRose 2011). A higher correlation between drug response of patient-derived tumor xenografts and corresponding original tumors is expected allowing further development of more efficacious, individualized tumor therapies.

\section{References}

Alvarez JV, Perez D, Chodosh LA. "mILC-ing the mouse mammary gland: A model for invasive lobular carcinoma." Cancer Cell 2006 10: 347-349.

Anand S, Penrhyn-Lowe S, Venkitaraman AR. "AURORA-A amplification overrides the mitotic spindle assembly checkpoint, inducing resistance to Taxol." Cancer Cell. 2003 Jan;3(1):51-62.

Anderson K, Yang J, Koretke K, Nurse K, Calamari A, Kirkpatrick RB, Patrick D, Silva D, Tummino PJ, Copeland RA, Lai Z. "Binding of TPX2 to Aurora A alters substrate and inhibitor interactions." Biochemistry. 2007 Sep 11;46(36):10287-95. 
Aquino JB, Lallemend F, Marmigère F, Adameyko II, Golemis EA, Ernfors P. "The retinoic acid inducible Cas-family signaling protein Nedd9 regulates neural crest cell migration by modulating adhesion and actin dynamics." Neuroscience. 2009 Sep 15;162(4):1106-19.

Arlot-Bonnemains Y, Klotzbucher A, Giet R, Uzbekov R, Bihan R, Prigent C. "Identification of a functional destruction box in the Xenopus laevis aurora-A kinase pEg2." FEBS Lett. 2001 Nov 9;508(1):149-52.

Bargon SD, Gunning PW, O'Neill GM."The Cas family docking protein, HEF1, promotes the formation of neurite-like membrane extensions." Biochim Biophys Acta. 2005 Dec 15;1746(2):143-54.

Bayliss R, Sardon T, Vernos I, Conti E. "Structural basis of Aurora-A activation by TPX2 at the mitotic spindle.” Mol Cell. 2003 Oct;12(4):851-62.

Bischoff JR, Anderson L, Zhu Y, Mossie K, Ng L, Souza B, Schryver B, Flanagan P, Clairvoyant F, Ginther C, Chan CS, Novotny M, Slamon DJ, Plowman GD. "A homologue of Drosophila aurora kinase is oncogenic and amplified in human colorectal cancers." EMBO J. 1998 Jun 1;17(11):3052-65.

Bodvarsdottir SK, Hilmarsdottir H, Birgisdottir V, Steinarsdottir M, Jonasson JG, Eyfjord JE.

"Aurora-A amplification associated with BRCA2 mutation in breast tumours." Cancer Lett. 2007 Apr 8;248(1):96-102.

Borowsky AD. "Choosing a mouse model: experimental biology in context--the utility and limitations of mouse models of breast cancer." Cold Spring Harb Perspect Biol. 2011 Sep 1;3(9):a009670.

Bradbury P, Mahmassani M, Zhong J, Turner K, Paul A, Verrills NM, O'Neill GM. "PP2A phosphatase suppresses function of the mesenchymal invasion regulator NEDD9." Biochim Biophys Acta. 2012 Feb;1823(2):290-7.

Bruna A, Greenwood W, Le Quesne J, Teschendorff A, Miranda-Saavedra D, Rueda OM, Sandoval JL, Vidakovic AT, Saadi A, Pharoah P, Stingl J, Caldas C. "TGF $\beta$ induces the formation of tumour-initiating cells in claudin low breast cancer." Nat Commun. 2012;3:1055.

Castro A, Vigneron S, Bernis C, Labbé JC, Prigent C, Lorca T. "The D-Box-activating domain (DAD) is a new proteolysis signal that stimulates the silent D-Box sequence of Aurora-A." EMBO Rep. 2002 Dec;3(12):1209-14.

Chang JX, Gao F, Zhao GQ, Zhang GJ. "Expression and clinical significance of NEDD9 in lung tissues." Med Oncol. 2012 Dec;29(4):2654-60.

Crane R, Kloepfer A, Ruderman JV. "Requirements for the destruction of human Aurora-A." J Cell Sci. 2004 Dec 1;117(Pt 25):5975-83.

Dadke D, Jarnik M, Pugacheva EN, Singh MK, Golemis EA."Deregulation of HEF1 impairs Mphase progression by disrupting the RhoA activation cycle." Mol Biol Cell. 2006 Mar;17(3):120417. 
de Jong $R$, van Wijk A, Haataja L, Heisterkamp N, Groffen J. "BCR/ABL-induced leukemogenesis causes phosphorylation of Hef1 and its association with Crkl." J Biol Chem. 1997 Dec 19;272(51):32649-55.

Derksen PW, Liu X, Saridin F, van der Gulden H, Zevenhoven J, Evers B, van Beijnum JR, Griffioen AW, Vink J, Krimpenfort P, Peterse JL, Cardiff RD, Berns A, Jonkers J. "Somatic inactivation of E-cadherin and p53 in mice leads to metastatic lobular mammary carcinoma through induction of anoikis resistance and angiogenesis." Cancer Cell. 2006 Nov;10(5):437-49.

DeRose YS, Wang G, Lin YC, Bernard PS, Buys SS, Ebbert MT, Factor R, Matsen C, Milash BA, Nelson E, Neumayer L, Randall RL, Stijleman IJ, Welm BE, Welm AL. "Tumor grafts derived from women with breast cancer authentically reflect tumor pathology, growth, metastasis and disease outcomes." Nat Med. 2011 Oct 23;17(11):1514-20.

Dilworth SM. "Polyoma virus middle $T$ antigen and its role in identifying cancer-related molecules." Nat Rev Cancer 2002 2: 951-956.

Eyers PA, Churchill ME, Maller JL. "The Aurora A and Aurora B protein kinases: a single amino acid difference controls intrinsic activity and activation by TPX2." Cell Cycle. 2005 Jun;4(6):7849.

Eyers PA, Erikson E, Chen LG, Maller JL. "A novel mechanism for activation of the protein kinase Aurora A." Curr Biol. 2003 Apr 15;13(8):691-7.

Floyd S, Pines J, Lindon C. "APC/C Cdh1 targets aurora kinase to control reorganization of the mitotic spindle at anaphase." Curr Biol. 2008 Nov 11;18(21):1649-58.

Gadea BB, Ruderman JV. "Aurora kinase inhibitor ZM447439 blocks chromosome-induced spindle assembly, the completion of chromosome condensation, and the establishment of the spindle integrity checkpoint in Xenopus egg extracts." Mol Biol Cell. 2005 Mar;16(3):1305-18.

Gavet O, Pines J. "Progressive activation of CyclinB1-Cdk1 coordinates entry to mitosis." Dev Cell. 2010 Apr 20;18(4):533-43.

Giampieri S, Manning C, Hooper S, Jones L, Hill CS, Sahai E. "Localized and reversible TGFbeta signalling switches breast cancer cells from cohesive to single cell motility." Nat Cell Biol. 2009 Nov;11(11):1287-96.

Giet R, Prigent C. "The non-catalytic domain of the Xenopus laevis auroraA kinase localises the protein to the centrosome." J Cell Sci. 2001 Jun;114(Pt 11):2095-104.

Gizatullin F, Yao Y, Kung V, Harding MW, Loda M, Shapiro GI. "The Aurora kinase inhibitor VX680 induces endoreduplication and apoptosis preferentially in cells with compromised p53dependent postmitotic checkpoint function." Cancer Res. 2006 Aug 1;66(15):7668-77.

Glover DM, Leibowitz MH, McLean DA, Parry H. "Mutations in aurora prevent centrosome separation leading to the formation of monopolar spindles." Cell. 1995 Apr 7;81(1):95-105.

Goepfert TM, Adigun YE, Zhong L, Gay J, Medina D, Brinkley WR."Centrosome amplification and overexpression of aurora A are early events in rat mammary carcinogenesis." Cancer Res. 2002 Jul 15;62(14):4115-22. 
Gritsko TM, Coppola D, Paciga JE, Yang L, Sun M, Shelley SA, Fiorica JV, Nicosia SV, Cheng $\mathrm{JQ}$. "Activation and overexpression of centrosome kinase BTAK/Aurora-A in human ovarian cancer." Clin Cancer Res. 2003 Apr;9(4):1420-6.

Guan Z, Wang XR, Zhu XF, Huang XF, Xu J, Wang LH, Wan XB, Long ZJ, Liu JN, Feng GK, Huang W, Zeng YX, Chen FJ, Liu Q. "Aurora-A, a negative prognostic marker, increases migration and decreases radiosensitivity in cancer cells." Cancer Res. 2007 Nov 1;67(21):10436-44.

Guy CT, Webster MA, Schaller M, Parsons TJ, Cardiff RD, Muller WJ. "Expression of the neu protooncogene in the mammary epithelium of transgenic mice induces metastatic disease." Proc Natl Acad Sci USA. 1992;89(22):10578-10582.

Harrington EA, Bebbington D, Moore J, Rasmussen RK, Ajose-Adeogun AO, Nakayama T, Graham JA, Demur C, Hercend T, Diu-Hercend A, Su M, Golec JM, Miller KM. "VX-680, a potent and selective small-molecule inhibitor of the Aurora kinases, suppresses tumor growth in vivo." Nat Med. 2004 Mar;10(3):262-7.

Heron NM, Anderson M, Blowers DP, Breed J, Eden JM, Green S, Hill GB, Johnson T, Jung $\mathrm{FH}$, McMiken HH, Mortlock AA, Pannifer AD, Pauptit RA, Pink J, Roberts NJ, Rowsell S.

"SAR and inhibitor complex structure determination of a novel class of potent and specific Aurora kinase inhibitors." Bioorg Med Chem Lett. 2006 Mar 1;16(5):1320-3.

Hirota T, Kunitoku N, Sasayama T, Marumoto T, Zhang D, Nitta M, Hatakeyama K, Saya H. "Aurora-A and an interacting activator, the LIM protein Ajuba, are required for mitotic commitment in human cells." Cell. 2003 Sep 5;114(5):585-98.

Hivert V, Pierre J, Raingeaud J. "Phosphorylation of human enhancer of filamentation (HEF1) on serine 369 induces its proteasomal degradation." Biochem Pharmacol. 2009 Oct 15;78(8):1017-25.

Hoar K, Chakravarty A, Rabino C, Wysong D, Bowman D, Roy N, Ecsedy JA. "MLN8054, a small-molecule inhibitor of Aurora A, causes spindle pole and chromosome congression defects leading to aneuploidy." Mol Cell Biol. 2007 Jun;27(12):4513-25.

Honda K, Mihara H, Kato Y, Yamaguchi A, Tanaka H, Yasuda H, Furukawa K, Urano T. "Degradation of human Aurora2 protein kinase by the anaphase-promoting complex-ubiquitinproteasome pathway." Oncogene. 2000 Jun 1;19(24):2812-9.

Horn V, Thélu J, Garcia A, Albigès-Rizo C, Block MR, Viallet J. "Functional interaction of Aurora-A and PP2A during mitosis." Mol Biol Cell. 2007 Apr;18(4):1233-41.

lida J, Dorchak J, Lehman JR, Clancy R, Luo C, Chen Y, Somiari S, Ellsworth RE, Hu H, Mural RJ, Shriver CD. "FH535 inhibited migration and growth of breast cancer cells." PLoS One. 2012;7(9):e44418.

Inamoto S, Iwata S, Inamoto T, Nomura S, Sasaki T, Urasaki Y, Hosono O, Kawasaki H, Tanaka H, Dang NH, Morimoto C."Crk-associated substrate lymphocyte type regulates transforming growth factor-beta signaling by inhibiting Smad6 and Smad7." Oncogene. 2007 Feb 8;26(6):893-904. 
Ismail HM. "Overexpression of s6 kinase 1 in brain tumours is associated with induction of hypoxia-responsive genes and predicts patients' survival.” . J Oncol. 2012;2012:416927.

Izumchenko E, Singh MK, Plotnikova OV, Tikhmyanova N, Little JL, Serebriiskii IG, Seo S, Kurokawa M, Egleston BL, Klein-Szanto A, Pugacheva EN, Hardy RR, Wolfson M, Connolly DC, Golemis EA. "NEDD9 promotes oncogenic signaling in mammary tumor development." Cancer Res. 2009 Sep 15;69(18):7198-206.

Jenkins DE, Hornig YS, Oei Y, Dusich J, Purchio T. "Bioluminescent human breast cancer cell lines that permit rapid and sensitive in vivo detection of mammary tumors and multiple metastases in immune deficient mice." Breast Cancer Res. 2005;7(4):R444-54.

Kamiguchi K, Tachibana K, Iwata S, Ohashi Y, Morimoto C. "Cas-L is required for beta 1 integrin-mediated costimulation in human Tcells." J Immunol. 1999 Jul 15;163(2):563-8.

Katayama H, Sasai K, Kawai H, Yuan ZM, Bondaruk J, Suzuki F, Fujii S, Arlinghaus RB, Czerniak BA, Sen S. "Phosphorylation by aurora kinase A induces Mdm2-mediated destabilization and inhibition of p53." Nat Genet. 2004 Jan;36(1):55-62.

Katayama H, Zhou H, Li Q, Tatsuka M, Sen S. "Interaction and feedback regulation between STK15/BTAK/Aurora-A kinase and protein phosphatase 1 through mitotic cell division cycle." J Biol Chem. 2001 Dec 7;276(49):46219-24.

Kim M, Gans JD, Nogueira C, Wang A, Paik JH, Feng B, Brennan C, Hahn WC, Cordon-Cardo C, Wagner SN, Flotte TJ, Duncan LM, Granter SR, Chin L. "Comparative oncogenomics identifies NEDD9 as a melanoma metastasis gene." Cell. 2006 Jun 30;125(7):1269-81.

Kim SH, Xia D, Kim SW, Holla V, Menter DG, Dubois RN."Human enhancer of filamentation 1 Is a mediator of hypoxia-inducible factor-1alpha-mediated migration in colorectal carcinoma cells." Cancer Res. 2010 May 15;70(10):4054-63.

Kitajima S, Kudo Y, Ogawa I, Tatsuka M, Kawai H, Pagano M, Takata T. "Constitutive phosphorylation of aurora-a on ser51 induces its stabilization and consequent overexpression in cancer." PLoS One. 2007 Sep 26;2(9):e944.

Klein A, Jung V, Zang KD, Henn W, Montenarh M, Kartarius S, Steudel WI, Urbschat S. "Detailed chromosomal characterization of the breast cancer cell line MCF7 with special focus on the expression of the serine-threonine kinase 15." Oncol Rep. 2005 Jul;14(1):23-31.

Kondo S, Iwata S, Yamada T, Inoue Y, Ichihara H, Kichikawa Y, Katayose T, Souta-Kuribara A, Yamazaki H, Hosono O, Kawasaki H, Tanaka H, Hayashi Y, Sakamoto M, Kamiya K, Dang NH, Morimoto C."Impact of the integrin signaling adaptor protein NEDD9 on prognosis and metastatic behavior of human lung cancer." Clin Cancer Res. 2012 Nov 15;18(22):6326-38.

Kong C, Wang C, Wang L, Ma M, Niu C, Sun X, Du J, Dong Z, Zhu S, Lu J, Huang B."NEDD9 is a positive regulator of epithelial-mesenchymal transition and promotes invasion in aggressive breast cancer." PLoS One. 2011;6(7):e22666.

Kufer TA, Silljé HH, Körner R, Gruss OJ, Meraldi P, Nigg EA. "Human TPX2 is required for targeting Aurora-A kinase to the spindle." J Cell Biol. 2002 Aug 19;158(4):617-23. Epub 2002 Aug 12. 
Kyono WT, de Jong R, Park RK, Liu Y, Heisterkamp N, Groffen J, Durden DL. "Differential interaction of Crkl with Cbl or C3G, Hef-1, and gamma subunit immunoreceptor tyrosine-based activation motif in signaling of myeloid high affinity Fc receptor for lgG (Fc gamma RI)." J Immunol. 1998 Nov 15;161(10):5555-63.

Law SF, Estojak J, Wang B, Mysliwiec T, Kruh G, Golemis EA."Human enhancer of filamentation 1, a novel p130cas-like docking protein, associates with focal adhesion kinase and induces pseudohyphal growth in Saccharomyces cerevisiae." Mol Cell Biol. 1996 Jul;16(7):3327-37.

Law SF, O'Neill GM, Fashena SJ, Einarson MB, Golemis EA. "The docking protein HEF1 is an apoptotic mediator at focal adhesion sites.” Mol Cell Biol. 2000 Jul;20(14):5184-95.

Law SF, Zhang YZ, Fashena SJ, Toby G, Estojak J, Golemis EA. "Dimerization of the docking/adaptor protein HEF1 via a carboxy-terminal helix-loop-helix domain." Exp Cell Res. 1999 Oct 10;252(1):224-35.

Law SF, Zhang YZ, Klein-Szanto AJ, Golemis EA. "Cell cycle-regulated processing of HEF1 to multiple protein forms differentially targeted to multiple subcellular compartments." Mol Cell Biol. 1998 Jun;18(6):3540-51.

Lee EC, Frolov A, Li R, Ayala G, Greenberg NM. "Targeting Aurora kinases for the treatment of prostate cancer." Cancer Res. 2006 May 15;66(10):4996-5002.

Lee EYHP, Muller WJ. Oncogenes and tumor suppressor genes. Cold Spring Harb Perspect Biol. 2010. 2: a003236.

Li B, Rosen JM, McMenamin-Balano J, Muller WJ, Perkins AS. neu/ERBB2 cooperates with p53-172H during mammary tumorigenesis in transgenic mice. Mol Cell Biol. 1997;17(6):31553163.

Li D, Zhu J, Firozi PF, Abbruzzese JL, Evans DB, Cleary K, Friess H, Sen S. "Overexpression of oncogenic STK15/BTAK/Aurora A kinase in human pancreatic cancer." Clin Cancer Res. 2003 Mar;9(3):991-7.

Li Y, Bavarva JH, Wang Z, Guo J, Qian C, Thibodeau SN, Golemis EA, Liu W. "HEF1, a novel target of Wnt signaling, promotes colonic cell migration and cancer progression." Oncogene. 2011 Jun 9;30(23):2633-43.

Littlepage LE and Ruderman JV. "Identification of a new APC/C recognition domain, the A box, which is required for the Cdh1-dependent destruction of the kinase Aurora-A during mitotic exit" Genes Dev. 2002 September 1; 16(17): 2274-2285.

Liu Q, Kaneko S, Yang L, Feldman RI, Nicosia SV, Chen J, Cheng JQ. "Aurora-A abrogation of p53 DNA binding and transactivation activity by phosphorylation of serine 215." J Biol Chem. 2004 Dec 10;279(50):52175-82.

Liu X, Elia AE, Law SF, Golemis EA, Farley J, Wang T."A novel ability of Smad3 to regulate proteasomal degradation of a Cas family member HEF1." EMBO J. 2000 Dec 15;19(24):675969. 
Lucas JT Jr, Salimath BP, Slomiany MG, Rosenzweig SA. "Regulation of invasive behavior by vascular endothelial growth factor is HEF1-dependent." Oncogene. 2010 Aug 5;29(31):4449-59.

Mahadevan D, Bearss DJ, Vankayalapati H. "Structure-based design of novel anti-cancer agents targeting aurora kinases." Curr Med Chem Anticancer Agents. 2003 Jan;3(1):25-34.

Manié SN, Beck AR, Astier A, Law SF, Canty T, Hirai H, Druker BJ, Avraham H, Haghayeghi N, Sattler M, Salgia R, Griffin JD, Golemis EA, Freedman AS. "Involvement of p130(Cas) and p105(HEF1), a novel Cas-like docking protein, in a cytoskeleton-dependent signaling pathway initiated by ligation of integrin or antigen receptor on human B cells." J Biol Chem. 1997 Feb 14;272(7):4230-6.

Meraldi P, Honda R, Nigg EA. "Aurora-A overexpression reveals tetraploidization as a major route to centrosome amplification in p53-/- cells." EMBO J. 2002 Feb 15;21(4):483-92.

Miao Y, Li AL, Wang L, Fan CF, Zhang XP, Xu HT, Yang LH, Liu Y, Wang EH. "Overexpression of NEDD9 is associated with altered expression of E-Cadherin, $\beta$-Catenin and N-Cadherin and predictive of poor prognosis in non-small cell lung cancer." Pathol Oncol Res. 2013 Apr;19(2):281-6.

Minegishi M, Tachibana K, Sato T, Iwata S, Nojima Y, Morimoto C."Structure and function of Cas-L, a 105-kD Crk-associated substrate-related protein that is involved in beta 1 integrinmediated signaling in lymphocytes." J Exp Med. 1996 Oct 1;184(4):1365-75.

Moore SR, Persons DL, Sosman JA, Bobadilla D, Bedell V, Smith DD, Wolman SR, Tuthill RJ, Moon J, Sondak VK, Slovak ML."Detection of copy number alterations in metastatic melanoma by a DNA fluorescence in situ hybridization probe panel and array comparative genomic hybridization: a southwest oncology group study (S9431)." Clin Cancer Res. 2008 May 15;14(10):2927-35.

Muller WJ, Sinn E, Pattengale PK, Wallace R, Leder P. "Single-step induction of mammary adenocarcinoma in transgenic mice bearing the activated c-neu oncogene." Cell. 1988;54(1):105-115.

Nadler Y, Camp RL, Schwartz C, Rimm DL, Kluger HM, Kluger Y. "Expression of Aurora A (but not Aurora B) is predictive of survival in breast cancer." Clin Cancer Res. 2008 Jul 15;14(14):4455-62.

Nagashima T, Oyama M, Kozuka-Hata H, Yumoto N, Sakaki $\mathrm{Y}$, Hatakeyama M. "Phosphoproteome and transcriptome analyses of ErbB ligand-stimulated MCF-7 cells." Cancer Genomics Proteomics. 2008 May-Aug;5(3-4):161-8.

Namba R, Young LJ, Maglione JE, McGoldrick ET, Liu S, Wurz GT, DeGregorio MW, Borowsky AD, MacLeod CL, Cardiff RD, et al. 2005. Selective estrogen receptor modulators inhibit growth and progression of premalignant lesions in a mouse model of ductal carcinoma in situ. Breast Cancer Res 7: R881-R889.

Natarajan M, Stewart JE, Golemis EA, Pugacheva EN, Alexandropoulos K, Cox BD, Wang W, Grammer JR, Gladson CL. "HEF1 is a necessary and specific downstream effector of FAK that promotes the migration of glioblastoma cells." Oncogene. 2006 Mar 16;25(12):1721-32. 
Nourry C, Maksumova L, Pang M, Liu X, Wang T. "Direct interaction between Smad3, APC10, CDH1 and HEF1 in proteasomal degradation of HEF1." BMC Cell Biol. 2004 May 16;5:20.

Nowakowski J, Cronin CN, McRee DE, Knuth MW, Nelson CG, Pavletich NP, Rogers J, Sang BC, Scheibe DN, Swanson RV, Thompson DA."Structures of the cancer-related Aurora-A, FAK, and EphA2 protein kinases from nanovolume crystallography." Structure. 2002 Dec;10(12):165967.

Ohashi S, Sakashita G, Ban R, Nagasawa M, Matsuzaki H, Murata Y, Taniguchi H, Shima H, Furukawa K, Urano T. "Phospho-regulation of human protein kinase Aurora-A: analysis using anti-phospho-Thr288 monoclonal antibodies." Oncogene. 2006 Dec 14;25(59):7691-702.

Ohashi Y, Iwata S, Kamiguchi K, Morimoto C."Tyrosine phosphorylation of Crk-associated substrate lymphocyte-type is a critical element in TCR- and beta 1 integrin-induced T lymphocyte migration." J Immunol. 1999 Oct 1;163(7):3727-34.

Park $\mathrm{KH}$, Choi SE, Eom M, Kang Y. "Downregulation of the anaphase-promoting complex (APC)7 in invasive ductal carcinomas of the breast and its clinicopathologic relationships." Breast Cancer Res. 2005;7(2):R238-47.

Pugacheva EN, Golemis EA."The focal adhesion scaffolding protein HEF1 regulates activation of the Aurora-A and Nek2 kinases at the centrosome." Nat Cell Biol. 2005 Oct;7(10):937-46.

Pugacheva EN, Jablonski SA, Hartman TR, Henske EP, Golemis EA. "HEF1-dependent Aurora A activation induces disassembly of the primary cilium." Cell. 2007 Jun 29;129(7):1351-63.

Ratushny V, Pathak HB, Beeharry N, Tikhmyanova N, Xiao F, Li T, Litwin S, Connolly DC, Yen TJ, Weiner LM, Godwin AK, Golemis EA. "Dual inhibition of SRC and Aurora kinases induces postmitotic attachment defects and cell death." Oncogene. 2012 Mar 8;31(10):1217-27.

Reichardt W, Jung V, Brunner C, Klein A, Wemmert S, Romeike BF, Zang KD, Urbschat S.

"The putative serine/threonine kinase gene STK15 on chromosome 20q13.2 is amplified in human gliomas." Oncol Rep. 2003 Sep-Oct;10(5):1275-9.

Sanz-Moreno V, Gadea G, Ahn J, Paterson H, Marra P, Pinner S, Sahai E, Marshall CJ. "Rac activation and inactivation control plasticity of tumor cell movement." Cell. 2008 Oct 31;135(3):510-23.

Sasai K, Parant JM, Brandt ME, Carter J, Adams HP, Stass SA, Killary AM, Katayama H, Sen S. "Targeted disruption of Aurora A causes abnormal mitotic spindle assembly, chromosome misalignment and embryonic lethality." Oncogene. 2008 Jul 3;27(29):4122-7.

Shi Y, Reiman T, Li W, Maxwell CA, Sen S, Pilarski L, Daniels TR, Penichet ML, Feldman R, Lichtenstein A. "Targeting aurora kinases as therapy in multiple myeloma." Blood. 2007 May 1;109(9):3915-21.

Sima N, Cheng X, Ye F, Ma D, Xie X, Lü W. "The Overexpression of Scaffolding Protein NEDD9 Promotes Migration and Invasion in Cervical Cancer via Tyrosine Phosphorylated FAK and SRC." PLoS One. 2013 Sep 18;8(9):e74594. 
Singh MK, Izumchenko E, Klein-Szanto AJ, Egleston BL, Wolfson M, Golemis EA. "Enhanced genetic instability and dasatinib sensitivity in mammary tumor cells lacking NEDD9." Cancer Res. 2010 Nov 1;70(21):8907-16.

Soncini C, Carpinelli $P$, Gianellini L, Fancelli D, Vianello $P$, Rusconi L, Storici $P$, Zugnoni $P$, Pesenti E, Croci V, Ceruti R, Giorgini ML, Cappella P, Ballinari D, Sola F, Varasi M, Bravo R, Moll J. "PHA-680632, a novel Aurora kinase inhibitor with potent antitumoral activity." Clin Cancer Res. 2006 Jul 1;12(13):4080-9.

Speranza MC, Frattini V, Pisati F, Kapetis D, Porrati P, Eoli M, Pellegatta S, Finocchiaro G. "NEDD9, a novel target of miR-145, increases the invasiveness of glioblastoma." Oncotarget. 2012 Jul;3(7):723-34.

Sun C, Newbatt Y, Douglas L, Workman P, Aherne W, Linardopoulos S. "High-throughput screening assay for identification of small molecule inhibitors of Aurora2/STK15 kinase." J Biomol Screen. 2004 Aug;9(5):391-7.

Taguchi Si, Honda K, Sugiura K, Yamaguchi A, Furukawa K, Urano T. "Degradation of human Aurora-A protein kinase is mediated by hCdh1." FEBS Lett. 2002 May 22;519(1-3):59-65.

Tanaka T, Kimura M, Matsunaga K, Fukada D, Mori H, Okano Y. "Centrosomal kinase AlK1 is overexpressed in invasive ductal carcinoma of the breast." Cancer Res. 1999 May 1;59(9):2041-4.

Tao Y, Zhang P, Frascogna V, Lecluse Y, Auperin A, Bourhis J, Deutsch E. "Enhancement of radiation response by inhibition of Aurora-A kinase using siRNA or a selective Aurora kinase inhibitor PHA680632 in p53-deficient cancer cells." Br J Cancer. 2007 Dec 17;97(12):1664-72.

Tatsuka M, Katayama H, Ota T, Tanaka T, Odashima S, Suzuki F, Terada Y. "Multinuclearity and increased ploidy caused by overexpression of the aurora- and Ipl1-like midbody-associated protein mitotic kinase in human cancer cells." Cancer Res. 1998 Nov 1;58(21):4811-6.

Tikhmyanova N, Golemis EA."NEDD9 and BCAR1 negatively regulate E-cadherin membrane localization, and promote E-cadherin degradation." PLoS One. 2011;6(7):e22102.

Tyler RK, Shpiro N, Marquez R, Eyers PA. "VX-680 inhibits Aurora A and Aurora B kinase activity in human cells." Cell Cycle. 2007 Nov 15;6(22):2846-54.

Walter AO, Seghezzi W, Korver W, Sheung J, Lees E. "The mitotic serine/threonine kinase Aurora2/AlK is regulated by phosphorylation and degradation." Oncogene. 2000 Oct 5;19(42):4906-16.

Wang LH, Xiang J, Yan M, Zhang Y, Zhao Y, Yue CF, Xu J, Zheng FM, Chen JN, Kang Z, Chen TS, Xing D, Liu Q. "The mitotic kinase Aurora-A induces mammary cell migration and breast cancer metastasis by activating the Cofilin-F-actin pathway." Cancer Res. 2010 Nov 15;70(22):9118-28.

Wang X, Zhou YX, Qiao W, Tominaga Y, Ouchi M, Ouchi T, Deng CX. "Overexpression of aurora kinase $A$ in mouse mammary epithelium induces genetic instability preceding mammary tumor formation". Oncogene. 2006 Nov 16;25(54):7148-58. 
Warner SL, Bashyam S, Vankayalapati H, Bearss DJ, Han H, Mahadevan D, Von Hoff DD, Hurley LH. "Identification of a lead small-molecule inhibitor of the Aurora kinases using a structure-assisted, fragment-based approach." Mol Cancer Ther. 2006 Jul;5(7):1764-73.

Warner SL, Munoz RM, Stafford P, Koller E, Hurley LH, Von Hoff DD, Han H.

"Comparing Aurora A and Aurora B as molecular targets for growth inhibition of pancreatic cancer cells." Mol Cancer Ther. 2006 Oct;5(10):2450-8.

Wu JC, Chen TY, Yu CT, Tsai SJ, Hsu JM, Tang MJ, Chou CK, Lin WJ, Yuan CJ, Huang CY. "Identification of V23RalA-Ser194 as a critical mediator for Aurora-A-induced cellular motility and transformation by small pool expression screening." J Biol Chem. 2005 Mar 11;280(10):9013-22.

Van Seventer GA, Salmen HJ, Law SF, O'Neill GM, Mullen MM, Franz AM, Kanner SB, Golemis EA, van Seventer JM. "Focal adhesion kinase regulates beta1 integrin-dependent $T$ cell migration through an HEF1 effector pathway." Eur J Immunol. 2001 May;31(5):1417-27.

Xia D, Holla VR, Wang D, Menter DG, DuBois RN."HEF1 is a crucial mediator of the proliferative effects of prostaglandin $\mathrm{E}(2)$ on colon cancer cells." Cancer Res. 2010 Jan $15 ; 70(2): 824-31$.

Xia L, Huang W, Tian D, Zhu H, Qi X, Chen Z, Zhang Y, Hu H, Fan D, Nie Y, Wu K. "Overexpression of forkhead box $\mathrm{C} 1$ promotes tumor metastasis and indicates poor prognosis in hepatocellular carcinoma." Hepatology. 2013 Feb;57(2):610-24.

Xu J, Li H, Wang B, Xu Y, Yang J, Zhang X, Harten SK, Shukla D, Maxwell PH, Pei D, Esteban MA. VHL inactivation induces HEF1 and Aurora kinase A." J Am Soc Nephrol. 2010 Dec;21(12):2041-6.

Xue YZ, Sheng YY, Liu ZL, Wei ZQ, Cao HY, Wu YM, Lu YF, Yu LH, Li JP, Li ZS. "Expression of NEDD9 in pancreatic ductal adenocarcinoma and its clinical significance." Tumour Biol. 2013 Apr;34(2):895-9.

Yang WH, Lan HY, Huang CH, Tai SK, Tzeng CH, Kao SY, Wu KJ, Hung MC, Yang MH. "RAC1 activation mediates Twist1-induced cancer cell migration." Nat Cell Biol. 2012 Mar 11;14(4):36674.

Zhang D, Hirota T, Marumoto T, Shimizu M, Kunitoku N, Sasayama T, Arima Y, Feng L, Suzuki M, Takeya M, Saya H. "Cre-loxP-controlled periodic Aurora-A overexpression induces mitotic abnormalities and hyperplasia in mammary glands of mouse models." Oncogene. 2004 Nov 18;23(54):8720-30.

Zhang Y, Ni J, Huang Q, Ren W, Yu L, Zhao S. "Identification of the auto-inhibitory domains of Aurora-A kinase.” Biochem Biophys Res Commun. 2007 Jun 1;357(2):347-52.

Zhao B, Smallwood A, Yang J, Koretke K, Nurse K, Calamari A, Kirkpatrick RB, Lai Z.

"Modulation of kinase-inhibitor interactions by auxiliary protein binding: crystallography studies on Aurora A interactions with VX-680 and with TPX2." Protein Sci. 2008 Oct;17(10):1791-7.

Zheng M, McKeown-Longo PJ. "Regulation of HEF1 expression and phosphorylation by TGFbeta 1 and cell adhesion.” J Biol Chem. 2002 Oct 18;277(42):39599-608. 
Zheng M, McKeown-Longo PJ. "Cell adhesion regulates Ser/Thr phosphorylation and proteasomal degradation of HEF1.” J Cell Sci. 2006 Jan 1;119(Pt 1):96-103.

Zhou H, Kuang J, Zhong L, Kuo WL, Gray JW, Sahin A, Brinkley BR, Sen S. "Tumour amplified kinase STK15/BTAK induces centrosome amplification, aneuploidy and transformation." Nat Genet. 1998 Oct;20(2):189-93. 


\section{Study1: NEDD9 Depletion Destabilizes Aurora A Kinase and Heightens the Efficacy of Aurora A Inhibitors: Implications for Treatment of Metastatic Solid Tumors}

Ryan J. Ice ${ }^{4}$, Sarah L. McLaughlin ${ }^{4}$, Ryan H. Livengood ${ }^{3}$, Mark V. Culp ${ }^{2}$, Erik R. Eddy ${ }^{2}$, Alexey V. Ivanov ${ }^{1,4}$, and Elena N. Pugacheva ${ }^{1,4}$

Departments of ${ }^{1}$ Biochemistry, ${ }^{2}$ Statistics, ${ }^{3}$ Pathology, and ${ }^{4}$ Mary Babb Randolph Cancer

Center, West Virginia University School of Medicine, Morgantown, West Virginia

Published in Cancer Res. 2013 May 15;73(10):3168-80.

Corresponding author:

Elena N. Pugacheva,

Department of Biochemistry,

PO Box 9142, 1 Medical Center Drive,

West Virginia University School of Medicine,

Morgantown, WV 26506.

Phone: 304-293-5295

Fax: 304-293-4667

E-mail: epugacheva@hsc.wvu.edu 


\begin{abstract}
Aurora A kinase (AURKA) is overexpressed in $96 \%$ of human cancers and is considered an independent marker of poor prognosis. While the majority of tumors have elevated levels of AURKA protein, few have AURKA gene amplification, implying that posttranscriptional mechanisms regulating AURKA protein levels are significant. Here, we show that NEDD9, a known activator of AURKA, is directly involved in AURKA stability. Analysis of a comprehensive breast cancer tissue microarray revealed a tight correlation between the expression of both proteins, significantly corresponding with increased prognostic value. A decrease in AURKA, concomitant with increased ubiquitination and proteasome-dependent degradation, occurs due to depletion or knockout of NEDD9. Reexpression of wild-type NEDD9 was sufficient to rescue the observed phenomenon. Binding of NEDD9 to AURKA is critical for AURKA stabilization, as mutation of S296E was sufficient to disrupt binding and led to reduced AURKA protein levels. NEDD9 confers AURKA stability by limiting the binding of the cdh1-substrate recognition subunit of APC/C ubiquitin ligase to AURKA. Depletion of NEDD9 in tumor cells increases sensitivity to AURKA inhibitors. Combination therapy with NEDD9 short hairpin RNAs and AURKA inhibitors impairs tumor growth and distant metastasis in mice harboring xenografts of breast tumors. Collectively, our findings provide rationale for the use of AURKA inhibitors in treatment of metastatic tumors and predict the sensitivity of the patients to AURKA inhibitors based on NEDD9 expression.
\end{abstract}




\section{Introduction}

The serine/threonine kinase, AURKA, is a proto-oncoprotein that is overexpressed in most cancers (1-3). High AURKA expression is strongly associated with decreased survival and is an independent prognostic marker (4). AURKA overexpression disrupts the spindle checkpoint activated by paclitaxel or nocodazole, inducing resistance to these compounds (5). Inhibition or depletion of AURKA protein may therefore improve the survival of patients resistant to paclitaxel (5). While $94 \%$ of the primary invasive mammary carcinomas have elevated AURKA protein levels (6), only $13.6 \%$ show AURKA gene amplification (1, 3). Thus, posttranscriptional mechanisms of AURKA stabilization are important in breast cancer.

AURKA is polyubiquitinated by the anaphase-promoting complex/cyclosome (APC/C) complex and targeted for degradation by the proteasome (7). APC/C-dependent degradation of AURKA requires cdh1, which acts as a substrate recognition subunit for a number of mitotic proteins, including Plk1 and cyclin B. Overexpression of cdh1 reduces AURKA levels (8), whereas cdh1 knockdown or mutation of the AURKA cdh1-binding site results in elevated AURKA expression (7-9). AURKA is ubiquitinated through the recognition of a carboxyl-terminal D-box (destruction box) and an amino-terminal A-box, specific for the destruction of AURKA (10, 11). Phosphorylation of AURKA on Ser51 in the A-box inhibits cdh1-APC/C-mediated ubiquitination and consequent AURKA degradation (9).

Cancer cells express high levels of AURKA independently of a cell cycle, which suggests that there are additional mechanisms of AURKA stabilization. Recently, a number of proteins were documented to be involved in the regulation of AURKA stability either by direct deubiquitination of AURKA (12) or through interference with AURKA ubiquitination by APC/C (PUM2, TPX2, LIMK2; refs. 13-15.)

NEDD9 is a member of metastatic gene signature identified in breast adenocarcinomas and melanomas (16-18). NEDD9 is a cytoplasmic docking protein of the CAS family. NEDD9 regulates proliferation directly by binding to and activating AURKA (19). In nontransformed cells, activation of AURKA by NEDD9 in interphase is tightly controlled by a limited amount of NEDD9 in cytoplasm. Overexpression of NEDD9 leads to the activation of AURKA resulting in centrosomal amplification and aberrant mitosis (19). NEDD9 undergoes ubiquitination and proteasomal degradation by APC/C. Like typical APC/C substrates, NEDD9 has D-box motifs and cdh1 binds to a D-box located within the carboxyl-terminal domain $(20,21)$.

The strong link between increased AURKA expression and cancer progression has stimulated development of AURKA inhibitors for cancer therapy. PHA-680632 (22, 23), MLN8054, and MLN8237 $(24,25)$ are potent small-molecule inhibitors of AURKA activity. These 
compounds have significant antitumor activity in various animal tumor models with favorable pharmacokinetics (23). However, clinical trials with MLN8054 as a single agent failed to show tumor growth inhibition $(25,26)$. In the present study, using human breast cell lines and xenografts, we have identified NEDD9 as a critical regulator of AURKA protein stability and sensitivity to AURKA inhibitors. Depletion of NEDD9 via short hairpin RNAs (shRNA) decreases AURKA protein, sensitizes tumor cells to AURKA inhibitors, and eliminates metastasis in xenograft models of breast cancer. Combination therapy using NEDD9 shRNAs and AURKA inhibitors might prove to be an effective treatment strategy for solid tumors with NEDD9 overexpression. 


\section{Materials and Methods}

\section{Plasmids and reagents}

shRNAs, siRNAs against human NEDD9, AURKA, and control expressed in pGIPZ or in doxycycline-inducible pTRIPZ vectors (Thermo Fisher Scientific). Lentiviral particles were prepared as previously described (26). Wild-type, Ser296Ala-A, S296/298-AA, Ser296Glu-E, and S296/298-EE cDNAs of murine NEDD9 were subcloned into pLUTZ lentiviral vector under doxycycline-inducible promoter. pcDNA3.1-myc-Ubiquitin and pcDNA3.1-HA-NEDD9 were used for ubiquitination studies. Induction of shRNA or cDNA was done by addition of $1 \mu \mathrm{g} / \mathrm{mL}$ doxycyline.

\section{Cell lines and culture conditions}

The cell lines MDA-MB-231, BT-549, BT-20, ZR-75-1, MCF7, and MDA-MB-231-luc-D3H2LN (MDA-MB-231LN) expressing luciferase (Caliper Life Sciences) were purchased and authenticated by American Type Culture Collection. After infection (or transfection) of shRNAs (or siRNAs), cells were selected for puromycin resistance and tested by Western blotting.

\section{Protein stability studies}

Approximately $2 \times 107$ cells were plated; 12 hours later, fresh medium containing cycloheximide $(50 \mu \mathrm{g} / \mathrm{mL})$ or MG132 (10 $\mu \mathrm{mol} / \mathrm{L})$ was added for 12 hours. At indicated time intervals, cells were lysed in protein Triton-based cell lysis buffer (19) with ubiquitin aldehyde (1-2 $\mu \mathrm{mol} / \mathrm{L})$, protease inhibitors (Sigma).

\section{Cell-cycle analysis by flow cytometry}

The fluorescence-activated cell-sorting (FACS) analysis was done according to a previously published protocol (19). Cell-cycle distribution was analyzed by FACSCalibur equipped with Cell Quest software.

\section{Quantitative real-time PCR}

Quantitative PCR (qPCR: ref. 27) was carried out in an ABI 7500 Real-Time PCR Cycler and analyzed using Applied Biosystems SDS software.

\section{Immunohistochemical analysis}

High-density breast cancer tissue microarrays BR2082 (Supplementary Table S1) were collected with full donor consent. Immunohistochemical (IHC) procedures were done according to the manufacturer's recommendations (US Biomax Inc.) in duplicates. Manual scoring of 
staining intensity [negative $(0)$, weak $(1+)$, moderate $(2+)$, or strong $(3+)$ ], as well as location and cell types was completed by an independent pathologist from US Biomax, Inc. Each core was scanned by the Aperio Scanning System at $\times 20$. The total number of positive cells and the intensity of anti-NEDD9 staining were computed by Aperio ImageScope10.1 software based on the digital images taken from each core.

\section{Western blot procedure and antibodies}

Western blotting procedures were previously described (26). Primary antibodies included antiNEDD9 monoclonal antibody (mAb; 2G9; ref. 19), anti-NEDD9 (p55, custom made using NEDD9 1-394 aa as an antigen), anti- $\beta$-actin mAb, or anti-GAPDH (Sigma); anti-AURKA (BD Biosciences); anti-AURKA (AurA-N, custom made using 1-126 aa N-terminal fragment of AURKA), anti-phospho-T288 Aurora A (Cell Signaling); and anti-histone H3, and anti-phosphoSer10 histone H3 and anti-ubiquitin (Millipore, BD Biosciences). Blots were developed by the HyGLO HRP Detection Reagent (Denville Scientific, Inc.). Bands were digitized and quantified using a digital electrophoresis documentation and image analysis system (G-box, Syngene Corp.).

\section{Protein expression, GST pull-down, and immunoprecipitation}

In vitro pull-down and immunoprecipitation protocols were previously published $(19,26)$. Immunoprecipitation samples were incubated with anti-AURKA (AurA-N) or anti-NEDD9 (p55), immobilized on the A/G-protein Sepharose or 4B-Glutathione agarose for GST pull-down (G\&E Healthcare Life Sciences) at $4^{\circ} \mathrm{C}$, washed and resolved by SDS-PAGE. His-tagged cdh1 protein (Novus International, Inc.), 50 ng of recombinant AURKA and GST-HEF1 in AURKA buffer were used in the cdh1 titration pull-down.

\section{Animal studies}

NOD.Cg-Prkdcscid I/2rgtm1Wjl/SzJ (NSG) immunodeficient mice were purchased from the Jackson Laboratory (stock 5557). Animals were housed in the WVU Animal Facility (Morgantown, WV) under pathogen-free conditions; protocol approved by the Institutional Animal Care and Use Committee. Primary tumor and organs with metastases were collected, processed, and analyzed by the WVU Department of Pathology Tissue Bank Core Facility.

\section{Animal bioluminescence imaging}

Mice were injected with luciferase-expressing MDA-MB-231LN cells and imaged weekly for quantitative evaluation of tumor growth and dissemination. About $150 \mathrm{mg} / \mathrm{kg}$ d-luciferin (Caliper 
Life Sciences) was injected into the peritoneum. Images were obtained using the IVIS Lumina-II Imaging System and Living Image-4.0 software.

1. Mammary fat pad injections: For animal studies, cells were grown, trypsinized, resuspended in DPBS $(1 \times 107$ cells $/ \mathrm{mL})$, and $0.1 \mathrm{~mL}$ was injected into the fourth inguinal mammary gland of female mice 6 to 8 weeks of age and followed by bioluminescence imaging $(\mathrm{BLI})$ up to 6 weeks.

2. Tail vein injections: Males were intravenously injected with $1 \times 105$ cells and followed by BLI once a week for 2 to 3 weeks total. Total radiance of lungs was calculated at each time point in control and treated animals. Lungs were imaged, fixed in formalin at the end point of study, and analyzed for number and size of metastases by a pathologist.

\section{Tumor volume measurement}

Tumor size was assessed by Vevo2100 Micro-Ultrasound System. A 40 or $50 \mathrm{mHz}$ transducer was used, depending on the tumor size, and a 3-dimensional image (3D) was acquired with $0.051 \mathrm{~mm}$ between images. Using the integrated software, the images were reconstructed to create a $3 D$ image of the tumor.

\section{AURKA inhibitors application}

\section{Cell line studies.}

Cells were treated with MLN8054 (0-100 nmol/L) or PHA-680632 (0-400 nmol/L) inhibitors (Selleckchem) for 2 to 12 hours, disrupted in protein Triton-based cell lysis buffer (19) processed for Western blotting or immunofluorescence staining.

\section{Xenograft studies.}

Compound administration began (i) when primary tumors reached 150 to $200 \mathrm{~mm} 3$ in female mice or (ii) 24 hours postintravenous injection of tumor cells in male mice. MLN8237 is an improved analog of MLN8054 compound with increased stability suitable for in vivo studies. MLN8237 was dissolved in 10\% 2-hydroxypropyl-b-cyclodextrin, 1\% sodium bicarbonate in water. About $20 \mathrm{mg} / \mathrm{kg} /$ dose was administered via oral gavage twice daily for 4 days/week for 2 weeks. MLN8237 was tested against a placebo control consisting of drug vehicle.

\section{Statistical analysis}

Unpaired $t$ test, nonlinear regression, or 1- or 2-way ANOVA with Tukey's multiple comparisons were used for statistical analysis of the results. Experimental values were reported as SEM. 
Differences in mean values were considered significant at $P<0.05$. Rates of tumor growth were established by linear regression of the bioluminescence data with time and cohort membership as covariates. Statistical calculations were conducted using the GraphPad InStat software package. 


\section{Results}

Increased NEDD9 expression tightly correlates with expression of AURKA protein in breast cancer.

AURKA and NEDD9 are independently overexpressed in many human cancers (1-3, 16-18). We have previously shown that NEDD9 binds to and activates AURKA in cancer cells (19), and expression of AURKA alone was found to be an independent prognostic marker of poor survival. To determine whether the expression of both proteins could facilitate the diagnosis of certain types or stages of breast cancer, we conducted IHC staining for NEDD9 and AURKA in 120 cases of breast cancer. Cases screened from a tissue microarray consisted of 4 groups of progressive disease stages: (i) normal tissue, (ii) intraductal carcinoma (IC), (iii) invasive ductal carcinoma (IDC), and (iv) metastatic IDC (MIDC; Supplementary Table S1). Representative images of IHC staining for each group are shown in Fig. 1A. Statistical analysis of staining intensity suggests that NEDD9 and AURKA expression positively correlate. The lowest expression and intensity of either protein was found in normal tissue, whereas a 10- to 20-fold increase in expression was observed in tumor samples (Fig. 1B). Significant correlation between NEDD9 and AURKA expression was noted for all 4 evaluated tissue types (Fig. 1C). The Spearman correlation coefficients for the normal, IC, IDC, and MIDC groups are 0.85, 0.67, 0.63, and 0.59, respectively, indicating a positive correlation (Fig. 1C). A random forest fit of NEDD9 and AURKA positivity staining, which achieved an out-of-bag error rate of 0.508 , indicates that by using NEDD9 and AURKA positivity scores, one can double the predictive power over chance (Fig. 1D). To define the molecular mechanisms underlying NEDD9 and AURKA correlative expression profiles, we used a panel of human breast cancer cell lines where the levels of NEDD9 can be manipulated and controlled.

\section{Depletion of NEDD9 leads to dramatic decrease of AURKA protein in cells lines and animal models}

The expression profiles of AURKA and NEDD9 in a panel of human breast cancer cell lines followed a pattern similar to that observed in the tissue microarray analysis. Invasive MDAMB-231 (or highly invasive lymph node-derived MDA-MB-231LN), MDA-MB-453, and ZR-75-1 cell lines had the highest levels of expression of NEDD9 and AURKA, followed by BT-549, and noninvasive MCF7 and BT-20 lines (Fig. 2A). We next evaluated AURKA protein levels in mouse embryonic fibroblasts derived from NEDD9 knockout (KO) and wild-type (WT) animals. AURKA expression levels were reduced in KO cells compared with WT cells (Fig. 2B). Similar 
results were obtained by $\mathrm{IHC}$ analysis of tissue sections (Fig. 2C), suggesting that maximal expression of AURKA is dependent on NEDD9.

Depletion of NEDD9 by 2 different shRNAs or siRNAs reduced the levels of AURKA protein by $60 \%$ to $80 \%$ (Fig. 2D; Supplementary Fig. S1A). To determine whether the reduced levels of AURKA were due to transcriptional mechanisms or nonspecific siRNA depletion, we carried out qRT-PCR analysis for NEDD9 and AURKA. The NEDD9-targeting siRNAs did not affect the levels of AURKA mRNA (Supplementary Fig. S1B), indicating that NEDD9 regulates AURKA at the protein level. Moreover, we were able to restore AURKA protein levels in shNEDD9 cells via re-expression of doxycycline-inducible WT-NEDD9 cDNA (Fig. 2E).

Protein levels of NEDD9 and AURKA are tightly regulated during the cell cycle (28); therefore, we examined the effects of NEDD9 depletion on the cell cycle. FACS analysis of cells treated with siRNA targeting NEDD9 did not show significant difference in cell-cycle distribution when compared with siCon (Supplementary Fig. S1C). These results indicate that the decrease in AURKA protein level is NEDD9-dependent and is post-transcriptionally regulated.

\section{NEDD9 regulates the stability of AURKA}

To further evaluate how NEDD9 governs AURKA expression, we examined AURKA levels in shCon- and shNEDD9-MDA-MB-231LN cells treated with the protein synthesis inhibitor cycloheximide. Cycloheximide treatment led to an abrupt decrease in the amount of AURKA in shNEDD9 cells during the first 3 hours (Fig. 3A). shCon cells had elevated AURKA levels for 6 hours and followed NEDD9 protein decay dynamics (Fig. 3A). The half-life of AURKA was 6 and 3 hours in shCon and shNEDD9, respectively (Fig. 3B). The delayed decrease in AURKA

protein levels in control cells compared with shNEDD9 indicates that AURKA protein stability is dependent on NEDD9.

\section{Decreased AURKA protein in NEDD9-deficient cells is caused by enhanced proteasome- dependent degradation}

NEDD9 and AURKA undergo ubiquitination and proteasomal degradation in a cellcycle-dependent manner $(9,20)$. To test whether the decrease in AURKA protein levels is associated with increased proteasome-based degradation, cell lines with depleted NEDD9 were treated with the proteasome inhibitor MG132 (Fig. 3C). Inhibition of proteasomal activity restored levels of AURKA in shNEDD9 cells to that of control cells (Fig. 3C and D), suggesting that NEDD9 protects AURKA from ubiquitination- and proteasome-dependent degradation. To directly test this, AURKA was immunoprecipitated from shNEDD9 and control cells and 
analyzed by immunoblotting with anti-AURKA and antiubiquitin antibodies. Depletion of NEDD9 increased the amount of ubiquitinated AURKA (Fig. 3E and F). Similar results were obtained with original MDA-MB-231 cells. Moreover, reexpression of wild-type NEDD9 was able to rescue this phenotype and decrease ubiquitination of AURKA (Supplementary Fig. S1E and S1F). NEDD9-dependent decrease in AURKA ubiquitination could be caused by steric hindrance of bound NEDD9 or by titration of ubiquitination machinery components, as both proteins use the APC/C-cdh1 complex $(8,20)$. To distinguish between these 2 possibilities, the levels of other APC/C-cdh1 targets, including Plk1 and Cdk1, in shNEDD9 cells were evaluated by immunoblotting. No difference in Plk1 and Cdk1 expression was detected between shNEDD9 and controls cells (Supplementary Fig. S1D), indicating that NEDD9 specifically targets AURKA and does not affect stability of other APC/C-cdh1 substrates. We have previously shown that NEDD9 binds to the N-terminal domain of AURKA containing the A-box motif (19), which is required for cdh1 binding and ubiquitination by $\operatorname{APC} / C(7,8)$. To test this hypothesis, we used recombinant NEDD9, cdh1, and AURKA in in vitro GST pull-down assay and examined whether the presence of NEDD9 imposes its inhibitory action on cdh1 directly. We confirmed that NEDD9 was able to bind AURKA in vitro in the presence of excess cdh1 and titrated cdh1 from the complex with AURKA in a concentration-dependent manner (Fig. $3 G$ and $H$ ). Thus, the presence of NEDD9 potentially increases AURKA protein levels by protecting AURKA from binding cdh1 resulting in reduced AURKA ubiquitination and subsequent proteasomal degradation.

\section{NEDD9 binding to AURKA is necessary for protein stabilization}

Phosphorylation of NEDD9 at S296 and S298 by AURKA impedes formation of the NEDD9/AURKA complex (19). To determine the impact of NEDD9 binding on AURKA protein levels, individual and dual phosphorylation null S296A-(A), S296A/S298A-(AA) and mimetic S296E-(E), S296E/S298E-(EE) forms of NEDD9 were generated. Indicated mutants were transfected in HEK293T cells followed by immunoprecipitation of AURKA (Fig. 4A). Immunoprecipitation analysis showed a reduction in binding to AURKA by the NEDD9 phosphomimetic (E, EE) mutants, whereas phosphorylation null (A, AA) NEDD9 mutants showed increase in AURKA binding (Fig. 4A and B), in agreement with our previously published observations (19). The increase in binding by phosphorylation-null mutants in this setting is expected because of overexpression of NEDD9 in HEK293T cells and inability of AURKA/NEDD9-AA complex to dissociate. To test the ability of these mutants to rescue the levels of AURKA in shNEDD9 cells, the 2 NEDD9 mutants (AA, EE) were overexpressed in an 
inducible manner and AURKA levels were measured by Western blotting (Fig. 4C and D). Reexpression of the AA mutant was sufficient to restore the levels of AURKA protein, meanwhile, the EE mutant failed to restore the levels of AURKA to control levels due to its inability to bind AURKA. Therefore, binding of NEDD9 to AURKA is necessary to stabilize AURKA.

NEDD9 binding to AURKA decreases the efficacy of AURKA inhibitors in vitro and in in vivo xenografts

Because of the structural proximity of the ATP-binding pocket to the NEDD9-binding domain on AURKA (23), we hypothesized that NEDD9-AURKA binding could potentially interfere with binding of AURKA inhibitors, in addition to preventing cdh1 binding. MLN8054 and PHA-680632 are ATP-competitive AURKA inhibitors that are currently in phase II clinical trials that have shown some efficacy against hematopoietic malignancies but have minimal effects against solid tumors (29-31). To evaluate the impact of NEDD9 expression on therapeutic outcome, NEDD9 was knocked down in breast cancer cell lines with high NEDD9 expression before treatment with PHA-680632 or MLN8054. NEDD9 depletion increased the efficacy of both inhibitors (Fig. 5A), decreasing the IC50 of PHA-680632 from $150 \mathrm{nmol} / \mathrm{L}$ in control cells to 50 to $100 \mathrm{nmol} / \mathrm{L}$ in shNEDD9 cells (Fig. 5B), as determined by the amount of active phT288AURKA. Similar results were obtained with MLN8054, where the IC50 value decreased from $200 \mathrm{nmol} / \mathrm{L}$ (shCon) to $20 \mathrm{nmol} / \mathrm{L}$ (shNEDD9; Fig. 5C and D). Inhibition of AURKA function was further confirmed by analysis of histone H3 phosphorylation in treated shCon and shNEDD9 cells. Inhibition of AURKA leads to accumulation of cells in mitosis characterized by phosphorylation of histone $\mathrm{H} 3$ at Ser10 (Fig. 5B and D and Supplementary Fig. S2E). The concentration of PHA-680632 was limited to $400 \mathrm{nmol} / \mathrm{L}$ to avoid targeting AURKB, which might lead to decrease in phosphorylation of histone $\mathrm{H} 3$.

\section{NEDD9 depletion alone or in combination with AURKA inhibitors reduces tumor burden and lung metastasis}

To examine the validity of our findings in in vivo xenograft models of human breast cancer, we used MDA-MB-231LN cells and shRNAs targeting NEDD9 or control. Tumor cells were injected into the mammary fat pad of NSG female mice and the tumor growth was assessed using BLI (Fig. 6A and B). The original MDA-MB-231LN cell line was tested and showed similar NEDD9 expression, tumor growth, and metastasis kinetics when compared with MDA-MB-231LN-shCon cells (Fig. 6A and B and Supplementary Fig. S1F). On the basis of 
these results, we have concluded that the MDA-MB-231LN-shCon cell line is a proper control and we used it in the subsequent experiments. Depletion of NEDD9 alone reduced tumor burden by $15 \%$ to $20 \%$ (Fig. $6 \mathrm{~B}$ ) and reduced the number of metastases in lungs by $25 \%$ to $50 \%$ (Fig. 6D and E shCon-V, shN2-V and Supplementary Fig. S2A).

Next, we combined shNEDD9 and AURKA inhibitor (MLN8237, a more stable analog of MLN8054) to test whether the combination will increase the efficacy of MLN8237 against primary tumor and metastasis (Fig. 6C-E). Treatment was initiated when primary tumor volume reached 150 to $200 \mathrm{~mm}$, based on ultrasound measurements in each cohort (30). Representative images and quantification of tumor volume is shown in Supplementary Fig. S2B and S2C. Difference in the rates of tumor growth among the 4 groups was assessed by BLI and pathology measurements using linear regression analysis with time, cell line, and treatment as covariates. Application of MLN8237 alone did not lead to a decrease in primary tumor growth, but in combination with shNEDD9, efficacy was improved 2-fold (Fig. 6C). Surprisingly, treatment with MLN8237 alone significantly decreased the number of lung metastases with a 2fold greater response in shNEDD9-expressing cells (Fig. 6D and E; Supplementary Fig. S2D).

Next, we used intravenous injection of breast cancer cells in NSG male mice to determine the impact of AURKA inhibitor on colonization by circulating tumor cells. On the basis of BLI data (Fig. 7A-C) and study endpoint pathology reports on dissected lungs (Supplementary Fig. S2F), shNEDD9-expressing cells were extremely sensitive to MLN8237 and were not capable of initiating tumor growth in lungs when compared with vehicle-treated cells. In summary, depletion of NEDD9 sensitizes human xenografted tumors and circulating tumor cells to AURKA inhibitors and eliminates metastasis to the lungs. Collectively, our data suggest a model (Fig. 7D) in which overexpression of NEDD9 renders AURKA less susceptible to Cdh1-APC-mediated ubiquitination and binding of small-molecule ATP-competitive inhibitors, thus protecting it from degradation and drug applications. 


\section{Discussion}

Recent studies corroborate overexpression of NEDD9 specifically with breast cancer and melanoma metastasis (16-18). Our data indicate that NEDD9 expression levels correlate positively with oncogenic AURKA expression and activation. Both proteins were correlated with pathologic parameters in human breast cancer cell lines and breast cancer patient samples. Moreover, achieving $80 \%$ accuracy for predicting cancer stage invasiveness is possible when screening for expression of both proteins.

AURKA inhibitors have recently entered phase II clinical trials for cancer treatment. However, the best response achieved in advanced tumors is disease stabilization, as tumor regression has not been reported (29-32). The knowledge of the molecular factors that influence AURKA stability and, therefore, sensitivity and resistance to AURKA inhibitors remains limited. With a few exceptions, such as TPX2 and PUM2 $(13,14)$, the role of AURKA activators in the stability of AURKA and their impact on sensitivity to AURKA inhibitors is unknown.

We show here that NEDD9 is a critical component in AURKA activation and stability. Furthermore, the molecular mechanism by which NEDD9/AURKA signaling functions to increase breast tumor cell resistance to AURKA inhibitors has been elucidated. Overexpression of NEDD9 in breast cancer cells prevents proteolytic degradation of AURKA and results in upregulation of AURKA protein level. AURKA activation correlates with protein stabilization, which in turn directly depends upon APC/C complex and its substrate recognition subunit, cdh1. Direct binding of NEDD9 to AURKA hampers the ability of the APC/C complex to ubiquitinate AURKA by preventing cdh1 binding. A NEDD9-dependent increase in AURKA protein and activity is critical for G2-M transition. Interestingly, the majority of proteins activating and/or stabilizing AURKA reside in the nucleus (Bora, TPX2, Ajuba, etc.) or require prior modifications (TPX2/AURKA binding is stimulated by the GTPase Ran) including phosphorylation by AURKA for binding (33-36). Nevertheless, a NEDD9-driven increase in the total amount of AURKA would be less noticeable in mitosis due to the inactivity of cdh1 (8). The excess of NEDD9 protein might promote the binding of known AURKA partners such as Ajuba, TPX2, and Bora $(33,34)$ leading to increased loading of AURKA on microtubules and Plk1 activity (37) and cancer progression. Direct binding of NEDD9 at the N-terminal domain decreases the efficacy of AURKA inhibitors in cell culture and in mammary tumor xenografts. Deletion or mutation of NEDD9 dramatically decreases AURKA protein level and kinase activity. Phosphorylation of NEDD9 by AURKA at Ser296 serves as a negative feedback loop to regulate the levels of active AURKA. The abundance of NEDD9 in epithelial cancers and dephosphorylation by PP2A (21) creates a constant supply of unphosphorylated NEDD9 that would stabilize and activate 
AURKA. We found that depletion of NEDD9 reduces tumor cell proliferation and lung metastases of orthotopic human tumor xenografts. Finally, we have established that treatment with AURKA inhibitors is particularly efficient against metastasis. In combination with shNEDD9 RNAs, MLN8237 abolishes lung metastases from orthotopic xenograft models as well as in lung colonization assays. No significant changes in animal weight and no apparent toxicity were noticed, suggesting a favorable toxicity profile.

The correlation of AURKA and NEDD9 expression in cancer patient biopsies could be critical for diagnostic purposes. It could potentially be used to predict the sensitivity of these patients to AURKA inhibitors. In addition, our results advocate the development and investigation of new NEDD9-targeting compounds as a novel therapeutic strategy against metastatic breast cancer. 


\section{Acknowledgements}

The authors thank Erica Peterson, Sherry Skidmore, Lana Yoho, Drs. Michael Schaller and Scott Weed (WVU) for critically reading the manuscript and outstanding administrative support, Erica Golemis (FCCC) for tissue sections, IHC NEDD9 KO mice, and WVU Tissue Bank and Animal Facility. 


\section{References}

1. Zhou H, Kuang J, Zhong L, Kuo WL, Gray JW, Sahin A, et al. Tumour amplified kinase STK15/BTAK induces centrosome amplification, aneuploidy and transformation. Nat Genet 1998;20:189-93.

2. Sen S, Zhou H,White RA. A putative serine/threonine kinase encoding gene BTAK on chromosome $20 \mathrm{q} 13$ is amplified and overexpressed in human breast cancer cell lines. Oncogene 1997;14:2195-200.

3. Bischoff JR, Anderson L, Zhu Y, Mossie K, Ng L, Souza B, et al. A homologue of Drosophila aurora kinase is oncogenic and amplified in human colorectal cancers. EMBO $J$ 1998;17:3052-65.

4. Nadler Y, Camp RL, Schwartz C, Rimm DL, Kluger HM, Kluger Y. Expression of Aurora A (but not Aurora B) is predictive of survival in breast cancer. Clin Cancer Res 2008;14:445562.

5. Agnese V, Bazan V, Fiorentino FP, Fanale D, Badalamenti G, Colucci G, et al. The role of Aurora-A inhibitors in cancer therapy. Ann Oncol 2007;18:vi47-52.

6.Tanaka T, Kimura M, Matsunaga K, Fukada D, Mori H, Okano Y. Centrosomal kinase AlK1 is overexpressed in invasive ductal carcinoma of the breast. Cancer Res 1999;59:2041-4.

7. Taguchi Si, Honda K, Sugiura K, Yamaguchi A, Furukawa K, Urano T. Degradation of human Aurora-A protein kinase is mediated by hCdh1. FEBS Lett 2002;519:59-65.

8. Floyd S, Pines J, Lindon C. APC/C(Cdh1) targets aurora kinase to control reorganization of the mitotic spindle at anaphase. Curr Biol 2008;18:1649-58.

9. Crane R, Kloepfer A, Ruderman JV. Requirements for the destruction of human Aurora-A. J Cell Sci 2004;117:5975-83.

10. Tyers M, Jorgensen P. Proteolysis and the cell cycle: with this RING I do thee destroy. Curr Opin Genet Dev 2000;10:54-64.

11. Zachariae W. Progression into and out of mitosis. Curr Opin Cell Biol 1999;11:708-16.

12. Shi Y, Solomon LR, Pereda-Lopez A, Giranda VL, Luo Y, Johnson EF, et al. Ubiquitinspecific cysteine protease 2a (USP2a) regulates the stability of Aurora-A. J Biol Chem 2011;286:38960-8.

13. Giubettini M, Asteriti IA, Scrofani J, De Luca M, Lindon C, Lavia P, et al. Control of Aurora-A stability through interaction with TPX2. J Cell Sci 2011;124:113-22.

14. Huang $\mathrm{YH}$, Wu CC, Chou CK, Huang CY. A translational regulator, PUM2, promotes both protein stability and kinase activity of Aurora-A. PLoS ONE 2011;6:e19718. 
15. Johnson EO, Chang KH, Ghosh S, Venkatesh C, Giger K, Low PS, et al. LIMK2 is a crucial regulator and effector of Aurora-A-kinase-mediated malignancy. J Cell Sci 2012;125:1204-16.

16. Minn AJ, Gupta GP, Siegel PM, Bos PD, Shu W, Giri DD, et al.Genes that mediate breast cancer metastasis to lung. Nature 2005;436:518-24.

17. Minn AJ, Gupta GP, Padua D, Bos P, Nguyen DX, Nuyten D, et al. Lung metastasis genes couple breast tumor size and metastatic spread. Proc Natl Acad Sci U S A 2007; 104:6740-5.

18. Kim M, Gans JD, Nogueira C, Wang A, Paik JH, Feng B, et al. Comparative oncogenomics identifies NEDD9 as a melanoma metastasis gene. Cell 2006;125:1269-81.

19. Pugacheva EN, Golemis EA. The focal adhesion scaffolding protein HEF1 regulates activation of the Aurora-A and Nek2 kinases at the centrosome. Nat Cell Biol 2005;7:93746.

20. Nourry C, Maksumova L, Pang M, Liu X, Wang T. Direct interaction between Smad3, APC10, CDH1 and HEF1 in proteasomal degradation of HEF1. BMC Cell Biol 2004;5:20.

21. Zheng M, McKeown-Longo PJ. Cell adhesion regulates Ser/Thr phosphorylation and proteasomal degradation of HEF1. J Cell Sci 2006;119:96-103.

22. Carpinelli P, Ceruti R, Giorgini ML, Cappella P, Gianellini L, Croci V, et al. PHA-739358, a potent inhibitor of Aurora kinases with a selective target inhibition profile relevant to cancer. Mol Cancer Ther 2007;6:3158-68.

23. Soncini C, Carpinelli P, Gianellini L, Fancelli D, Vianello P, Rusconi L, et al. PHA680632, a novel Aurora kinase inhibitor with potent antitumoral activity. Clin Cancer Res 2006;12:4080-9.

24. Hoar K, Chakravarty A, Rabino C, Wysong D, Bowman D, Roy N, et al.MLN8054, a small-molecule inhibitor of Aurora A, causes spindle pole and chromosome congression defects leading to aneuploidy. Mol Cell Biol 2007;27:4513-25.

25. Dees EC, Infante JR, Cohen RB, O'Neil BH, Jones S, von Mehren M, et al. Phase 1 study of MLN8054, a selective inhibitor of Aurora A kinase in patients with advanced solid tumors. Cancer Chemother Pharmacol 2011;67:945-54.

26. Pugacheva EN, Jablonski SA, Hartman TR, Henske EP, Golemis EA. HEF1-dependent Aurora A activation induces disassembly of the primary cilium. Cell 2007;129:1351-63.

27. Ruijter JM, Pfaffl MW, Zhao S, Spiess AN, Boggy G, Blom J, et al. Evaluation of qPCR curve analysis methods for reliable biomarker discovery: bias, resolution, precision, and implications. Methods 2013;59:32-46.

28. Pugacheva EN, Golemis EA. HEF1-aurora A interactions: points of dialog between the cell cycle and cell attachment signaling networks. Cell Cycle 2006;5:384-91. 
29. Maris JM, Morton CL, Gorlick R, Kolb EA, Lock R, Carol H, et al. Initial testing of the aurora kinase A inhibitor MLN8237 by the Pediatric Preclinical Testing Program (PPTP). Pediatr Blood Cancer 2010;55:26-34.

30. Manfredi MG, Ecsedy JA, Chakravarty A, Silverman L, Zhang M, Hoar KM, et al. Characterization of Alisertib (MLN8237), an investigational small-molecule inhibitor of aurora A kinase using novel in vivo pharmacodynamic assays. Clin Cancer Res 2011;17:7614-24.

31. Manfredi MG, Ecsedy JA, Meetze KA, Balani SK, Burenkova O, Chen W, et al. Antitumor activity of MLN8054, an orally active small-molecule inhibitor of Aurora A kinase. Proc Natl Acad Sci U S A 2007;104:4106-11.

32. Kasap E, Boyacioglu SO, Korkmaz M, Yuksel ES, Unsal B, Kahraman E, et al. Aurora kinase A (AURKA) and never in mitosis gene A-related kinase 6 (NEK6) genes are upregulated in erosive esophagitis and esophageal adenocarcinoma. Exp Ther Med 2012;4:33-42.

33. Hirota T, Kunitoku N, Sasayama T, Marumoto T, Zhang D, Nitta M, et al. Aurora-A and an interacting activator, the LIM protein Ajuba, are required for mitotic commitment in human cells. Cell 2003;114:585-98.

34. Hutterer A, Berdnik D, Wirtz-Peitz F, Zigman M, Schleiffer A, Knoblich JA. Mitotic activation of the kinase Aurora-A requires its binding partner Bora. Dev Cell 2006;11:14757.

35. Tsai MY, Wiese C, Cao K, Martin O, Donovan P, Ruderman J, et al. A Ran signalling pathway mediated by the mitotic kinase Aurora A in spindle assembly. Nat. Cell Biol 2003;5:242-8.

36. Chan EH, Santamaria A, Silljé HW, Nigg EA. Plk1 regulates mitotic Aurora A function through $\beta$ TrCP-dependent degradation of hBora. Chromosoma 2008;117:457-69.

37. Macurek L, Lindqvist A, Medema RH. Aurora-A and hBora join the game of Polo. Cancer Res 2009;69:4555-8. 


\section{Figure Legends}

Figure 1. Increased NEDD9 expression correlates with expression of AURKA protein in invasive ductal breast adenocarcinomas. A, representative images of IHC staining of tissue microarray with anti-AURKA (top) and anti-NEDD9 (bottom) antibodies. Normal breast tissue, intraductal carcinoma (IC), invasive ductal carcinoma (IDC), metastatic invasive ductal carcinoma, lymph nodes (MIDC-LN). Scale bar, $200 \mu \mathrm{m}$. Insets represent $\times 200$ enlarged areas indicated in the main panel. B, quantification of NEDD9 and AURKA in relative units of positivity (RUP; percentage of cells stained positively in the same core stained by anti-AURKA or NEDD9 antibodies). Most fitted lines were plotted on the graphs. C, positivity data for both proteins were analyzed using Spearman Rho correlation. D, positivity data for both proteins were analyzed by random forest statistical software.

Figure 2. Depletion of NEDD9 leads to dramatic decrease of AURKA protein. Western blot analysis of NEDD9 and AURKA in breast cancer cell lines (A) and mouse embryonic fibroblasts (B) derived from wild-type (WT) and NEDD9 knock out (KO) animals. C, representative IHC of anti-AURKA staining in kidney tissues, WT, and NEDD9-KO mice. Staining with nonspecific lgG (Con.). Scale bar, $25 \mu \mathrm{m}$. D, Western blot analysis and quantification of AURKA, NEDD9, and glyceraldehyde-3-phosphate dehydrogenase (GAPDH) upon treatment with anti-NEDD9 (shN1, shN2) or nonspecific (shCon) shRNAs, $n=3$, percentage of AURKA expression to shCon, \pm SEM. Student one-tailed $t$ test: ${ }^{* *}, P=0.0006 ;{ }^{* *}, P=0.0024$ (shCon/shN1 or/shN2) and no significant difference (ns; shN1/shN2) in MCF7. ${ }^{* *}, P=0.003 ;{ }^{* *}, P=0.0094$ (shCon/shN1 or/shN2) and ns (shN1/shN2) in MDA-MB-231. E, WB analysis and quantification of AURKA and NEDD9 expression in shNEDD9 and shCon cells transfected with WT-NEDD9 or controlRFP (red fluorescent protein) cDNA; $n=3$, percentage of AURKA expression to shCon, normalized by GAPDH. Student one-tailed $t$ test: *, $P=0.0186$; ${ }^{*}, P=0.0012$ (shCon/shN1 or/shN2), ns (shN1/shN2 or shN1/NEDD9 and shN2/NEDD9 reexpression).

Figure 3. NEDD9 regulates stability of AURKA through inhibition of proteasome-dependent degradation. A, Western blot analysis of AURKA and NEDD9 expression in shCon-, shNEDD9MDA-MB-231LN cells treated with cycloheximide. Expression was normalized by a-tubulin. B, quantification of AURKA as in $A, n=3$, fold of change of AURKA expression to time point 0 hours, \pm SEM, one-way ANOVA: ${ }^{* * *}, P<0.0001$; ${ }^{* *}, P=0.005$ (shCon/shN1 or/shN2) at each time point (except 0 hour). C, Western blot analysis of AURKA, NEDD9 in shCon, shNEDD9 cells treated with MG132 or vehicle. D, quantification of AURKA expression as in C (+MG132), 
$n=3$, fold of change to shCon, \pm SEM. Student $t$ test: ${ }^{* *}, P=0.0086 ;{ }^{* *}, P=0.0084$ (shCon/shN1 or/shN2). E, Western blot analysis of AURKA ubiquitination in WCL and immunoprecipitation (IP) from shCon or shNEDD9 cells transfected with pcDNA3-Myc-Ubiquitin, with MG132. F, quantification of AURKA ubiquitination as in $\mathbf{E}, \mathrm{IP}, n=3$, fold of change to shCon, \pm SEM. Student $t$ test: ${ }^{*}, P=0.0098 ;{ }^{*}, P=0.05$ (shCon/shN1 or/shN2). G, WB with anti-AURKA, anti-NEDD9, and anti-cdh1 antibodies. $\mathbf{H}$, quantification of AURKA using the following formula (AURKA-IP/AURKA-total) normalized to NEDD9 pull-down as in $\mathrm{G}, n=3$, plotted as AURKA ratio \pm SEM, *,$P<0.05$.

Figure 4. NEDD9 binding to AURKA is necessary for protein stabilization. A, Western blot analysis of AURKA and NEDD9 expression in WCL (left) or IP-GFP (right) from 293T cells transfected with pAcGFP-NEDD9-WT, AA, EE A, E, mutants, or GFP control. B, quantification of AURKA-colP as in A, $n=3$, plotted as percentage \pm SEM. AURKA-colP with wtNEDD9 assigned $100 \%$. Student $t$ test: ${ }^{* *}, P=0.0013 ;{ }^{*}, P=0.010 ;{ }^{* *}, P=0.00209$, ns (WT/AA or/EE or/A or/E, respectively). C, WB analysis of AURKA and NEDD9 expression in WCL of MDA-MB231 cells expressing doxycycline-inducible NEDD9-AA, -EE, or empty vector control. D, quantification of AURKA expression as in $\mathrm{C}, n=3$, relative intensity units (RIU) \pm SEM, normalized to actin. Two-way ANOVA: $P<0.0001$. Student $t$ test: ${ }^{* *}, P=0.0063,0.0061$ [AA/EE (+Dox, 24, 48 hours)].

Figure 5. NEDD9 binding to AURKA decreases the efficacy of AURKA inhibitors in vitro. A, Western blot analysis of AURKA, phT288-AURKA, NEDD9 in shCon or shNEDD9-MDA-MB231LN cells treated with PHA-680632. B, quantification of phT288-AURKA expression as in A, $n$ $=3$, normalized to total AURKA and plotted as relative intensity units (RIU) \pm SEM. Two-way ANOVA: $P<0.0001$, (shCon/shN1 or shN2), $P=0.0013$ (shN1/shN2). Quantification of phS10Histone H3 (IF), $n=3,1000$ cells/treatment, $P=0.006, P=0.0067$ (shCon/shN1 or shN2), no significant difference (shN1/shN2; C). Western blot analysis of total AURKA, phT288-AURKA, NEDD9 expression in cells treated with increasing concentrations of MLN8054. D, quantification as in $\mathrm{B}$ for the Western blots shown in C. $P<0.0001$, (shCon/shN1 or shN2), $P=0.0041$ (shN1/shN2). Quantification of immunofluorescence shown in Supplementary Fig. S2E, $n=3$, 1,000 cells/treatment, $P=0.0053, P=0.0086$ (shCon/shN1 or shN2), no significant difference (shN1/shN2). 
Figure 6. Depletion of NEDD9 combined with AURKA inhibitors leads to decrease in a tumor burden and lung metastasis in breast cancer xenograft model. A, representative images of BLI of mice orthotopically injected with MDA-MB-231LN parental, shCon, or shNEDD9 (shN2) cells. $B$, quantification of BLI data as in A plotted as mean log photon flux \pm SEM. Linear regression analysis, $P=0.0021, P=0.0006$ (shCon/shN2), weeks 3 and 4 ; no significant difference (shCon/parental). C, quantification of BLI data, mice orthotopically injected with shNEDD9 or shCon cells and treated with MLN8237 (MLN) or vehicle, 3 independent experiments, $n=6$ in each group; mean photon flux, \pm SEM. Two-way ANOVA, $P=0.0061$ (shCon-MLN/shN2-MLN, days 7-11). D, representative images of $B L I$, lungs dissected from 4 groups. $E$, quantification of $\mathrm{BLI}$ data as in D, 3 independent experiments, $n=6$ /group; mean photon flux $\pm \mathrm{SEM}$. Two-way ANOVA: **, $P=0.0071$ (shCon-Vehicle/shN2-Vehicle); ${ }^{* *}, P=0.045$ (shCon-MLN/shN2-MLN).

Figure 7. Combination of AURKA Inhibitors with depletion of NEDD9 reduces colonization potential of human circulating tumor cells in lungs. A, representative images of BLI; mice intravenously injected with MDA-MB-231LN cells expressing shCon or shNEDD9 (shN2) and treated with MLN8237 (M) or vehicle (V). B, quantification of BLI data as in A, 3 independent experiments, $n=6 /$ group, plotted as fold growth of BLI mean radiance \pm SEM. Two-way ANOVA: $P<0.0001$ (shCon-V/shCon-M), (shN2-V/shN2-M), (shCon-V/shN2-V); $P=0.0145$ (shCon-M/shN2-M). C, quantification of $\mathrm{BLI}$ as in $\mathrm{A}$, dissected lungs; 3 independent experiments, $n=6 /$ group, mean photon flux \pm SEM. One-tailed Student $t$ test: $P<0.0001$ (shCon-V/shCon-M), (shN2-V/shN2-M), $P=0.009$ (shCon-V/shN2-V), $P=0.0335$ (shConM/shN-M). D, model of NEDD9 action on AURKA stability and inhibitors competitive binding (I/III). Under normal physiologic conditions with low NEDD9 expression, AURKA is not solely bound to NEDD9 and gets targeted by cdh1 and AURKA inhibitors (II/IV). Overexpression of NEDD9 leads to sequestering of AURKA by NEDD9 and limits cdh1 binding, thus stabilizing AURKA and hampers the ability AURKA inhibitors to access ATP pocket.

\section{Supplementary Figure 1. Depletion of NEDD9 with siRNA does not affect transcription or} cell cycle distribution, but decreases AURKA protein levels. (A). Western blot analysis of AURKA and NEDD9 protein levels in MCF7 cells transfected with siCon or siNEDD9 smart pool. Quantification of AURKA and NEDD9 proteins is shown on the right panel. (B). Quantitative PCR analyisis (qPCR) of NEDD9 and AURKA mRNA levels after siRNA treatment and in (A). One tailed Students t-test of aurka mRNA levels indicates no statistically significant difference (ns) between siNEDD9 and control, $p=0.327$ between siCon and siNEDD9. (C). Flow cytometry 
analysis of cell cycle distribution of siNEDD9 and siCon treated cells. (D). Western blot analysis of NEDD9, CDK1, cyclin B (CKB), PLK1 and actin expression levels in MDA-MB-231 cells expressing siControl or siNEDD9 (smart pool, 4 siRNAs). (E). WB analysis of NEDD9, AURKA and ubiquitinated AURKA in MDA-MB-231 cells expressing shCon, shNEDD9 (shN1) and shN1 rescued with exogenouse expression of wt HA-tagged NEDD9 cDNA. (F). WB analysis of NEDD9, AURKA and GAPDH in MDA-MB-231 and MDA-MB-231LN cells.

\section{Supplementary Figure 2. Combination of AURKA inhibitors with Depletion of NEDD9} dramatically reduces metastasis to lungs. (A). Quantification of number of metastasis in lungs of orthotopically injected animals at the end of the study. Results were plotted as mean number of metastases per $\mathrm{mm}^{2}$ +/-S.E.M from 3 independent experiments with $\mathrm{n}=6$ per treatment group. Two way ANOVA indicated statistically significant difference $p=0.048$ only for shCon-M and shN2-M, the rest was ns. (B). Representative images of Vevo200 ultrasound 3D reconstructed tumors from siNEDD9 (14 days) and siCon (8-10 days) orthotopically injected mice. Scale bar $1 \mathrm{~mm}$. (C). Quantification of tumor volume before application of MLN8237. Treatment was initiated upon similar volumes were reached in all 4 groups (time point zero for MLN treatment). One way ANOVA, did not identified any significant difference between the groups (ns). (D). Quantification of number of metastasis in lungs of intravenously injected animals at the end of the study of shCon and shNEDD9 (shN2) treated with MLN8237 (MLN) or vehicle alone at the end of the study. Results were plotted as average number of metastases per $\mathrm{mm}^{2}+$-S.E.M from 3 independent experiments with $\mathrm{n}=6$ per group. Two way ANOVA indicated statistically significant difference $\mathrm{p}=0.045$ only for shCon-M and shN2-M, the rest was ns. (E). Representative images of immunofluorescent staining with anti-phSer10-Histone H3 antibodies (magenta), DNA (dapi, cyan) and GIPZ-shRNAs (green) of shCon and shNEDD9 (shN1) MDA-MB-231LN cells. The scale bar is $10 \quad \square \mathrm{m}$. 
A
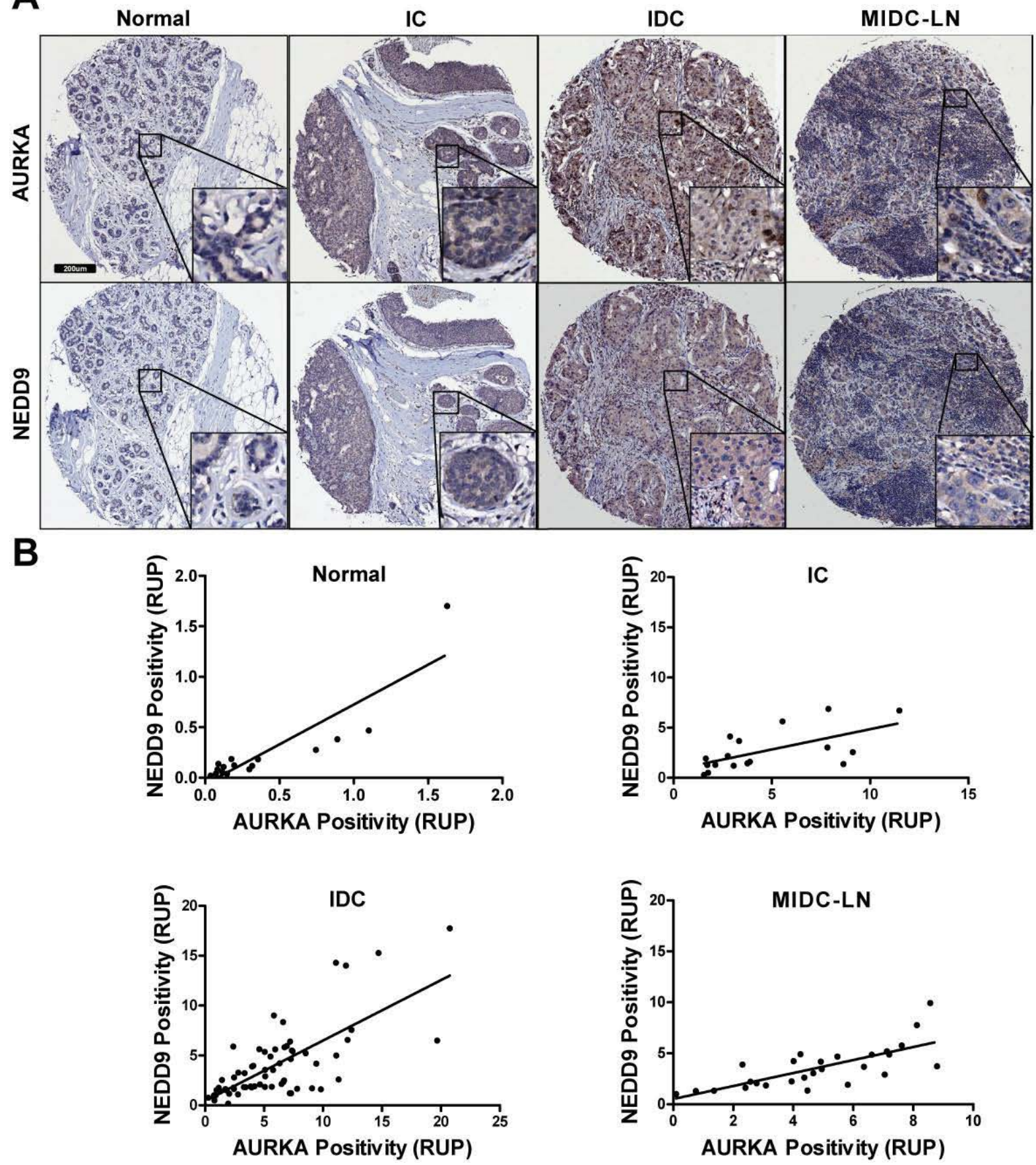

C

\begin{tabular}{|c|c|c|c|c|}
\cline { 2 - 5 } \multicolumn{1}{c|}{} & Normal & IC & IDC & MIDC-LN \\
\hline $\mathrm{N}=$ & 11 & 17 & 63 & 29 \\
\hline Spearman's Rho & 0.855 & 0.627 & 0.630 & 0.594 \\
\hline P value & 0.001 & 0.007 & $<0.001$ & 0.001 \\
\hline
\end{tabular}

Figure 1, Ice

\begin{tabular}{|c|c|c|c|c|c|c|}
\hline & & \multicolumn{4}{|c|}{ Diagnosed } & \multirow[b]{2}{*}{ Error Rate } \\
\hline & & Normal & IC & IDC & MIDC-LN & \\
\hline \multirow{4}{*}{ 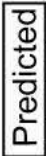 } & Normal & 10 & 0 & 1 & 0 & 0.09 \\
\hline & IC & 0 & 2 & 11 & 4 & 0.88 \\
\hline & IDC & 0 & 2 & 45 & 4 & 0.29 \\
\hline & MIDC-LN & 1 & 4 & 22 & 2 & 0.93 \\
\hline & & & & & Total Error & 0.50 \\
\hline
\end{tabular}




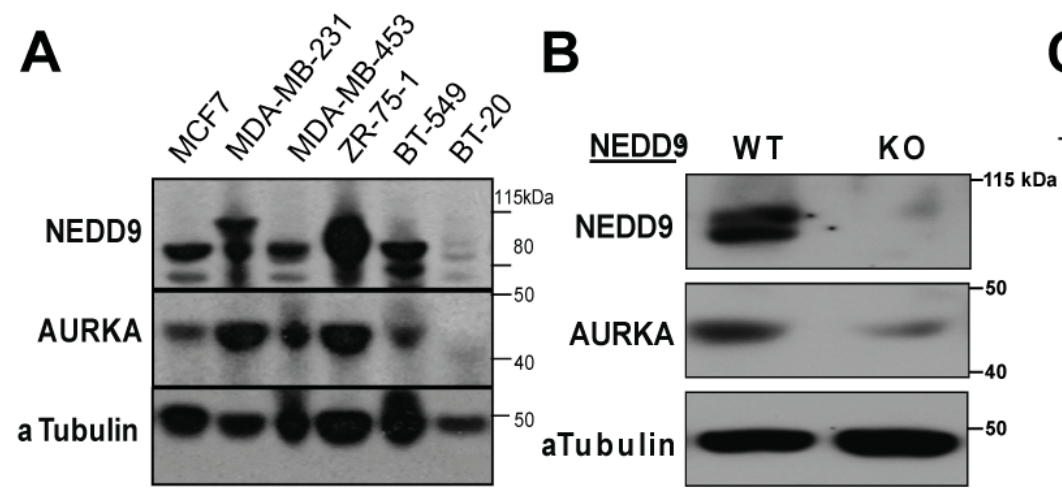

C

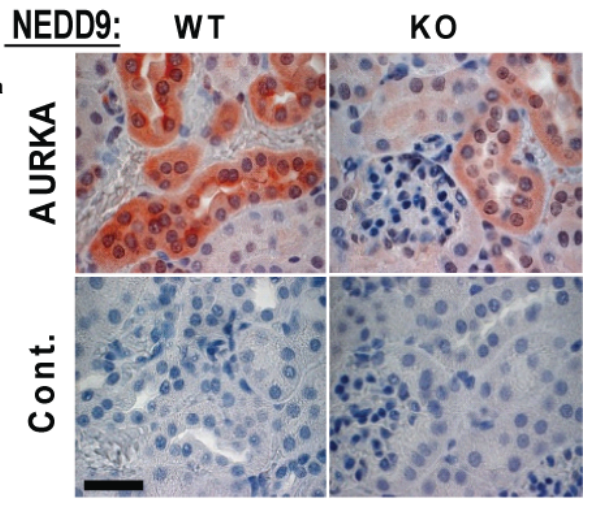

D
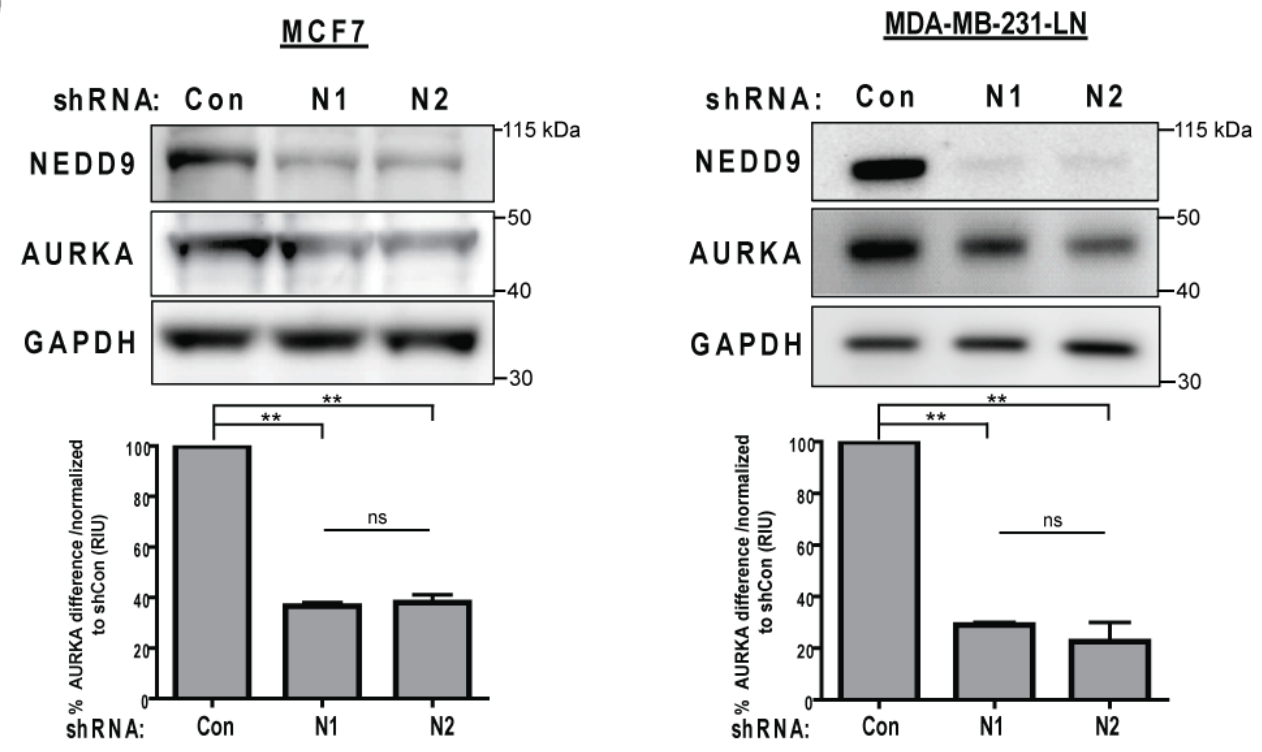

E

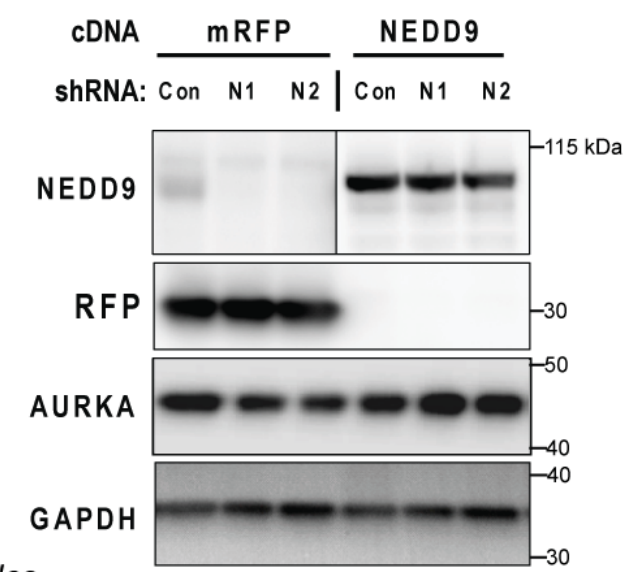

Figure 2, Ice

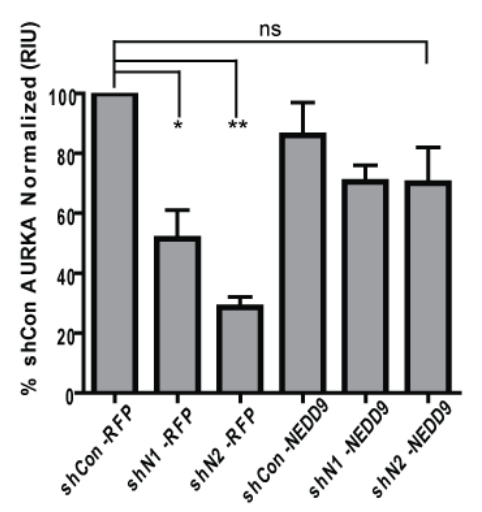


A
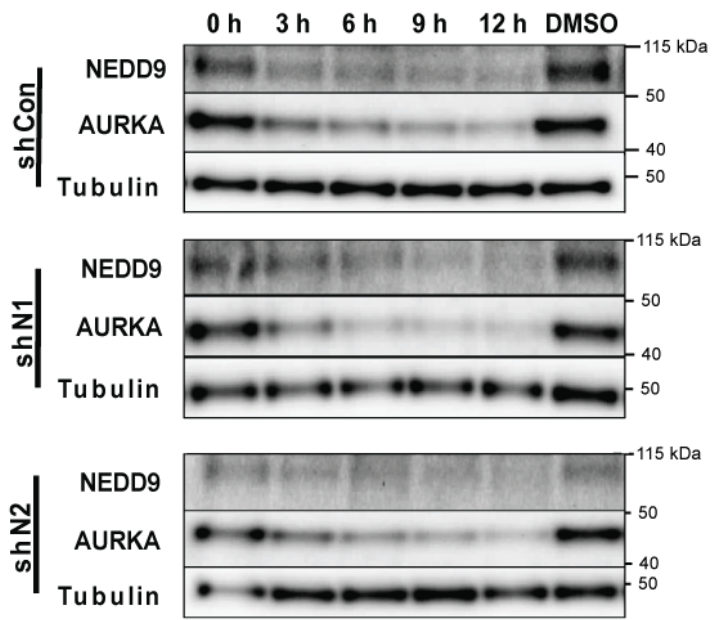

B

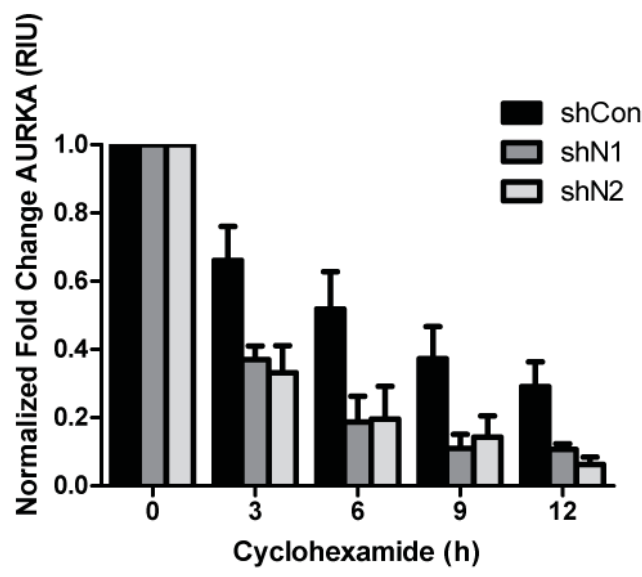

C

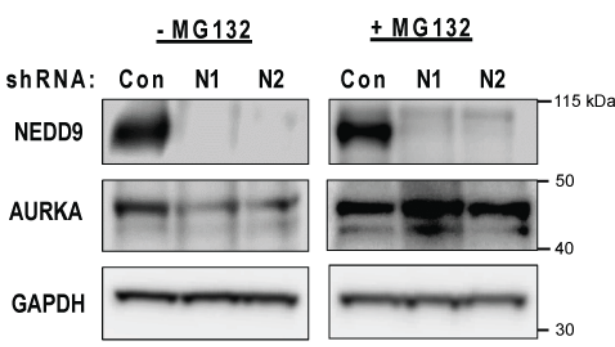

D

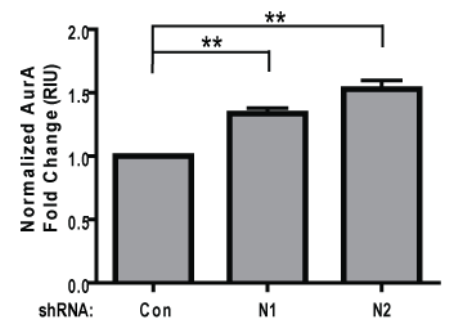

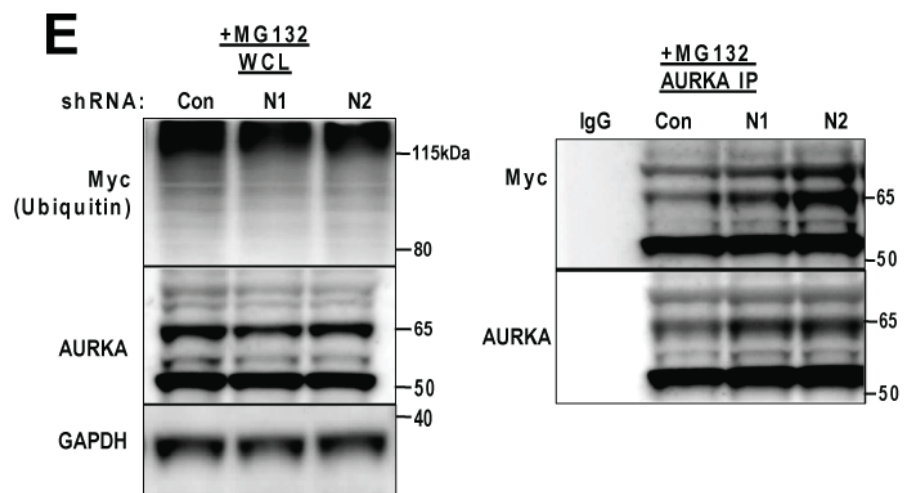

F

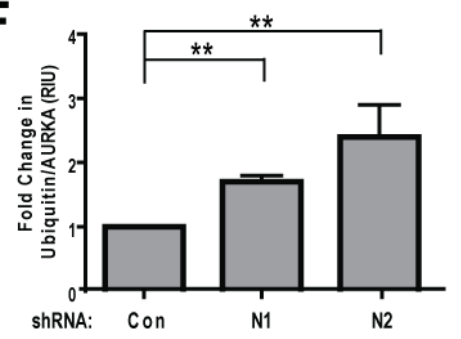

$\mathrm{H}$
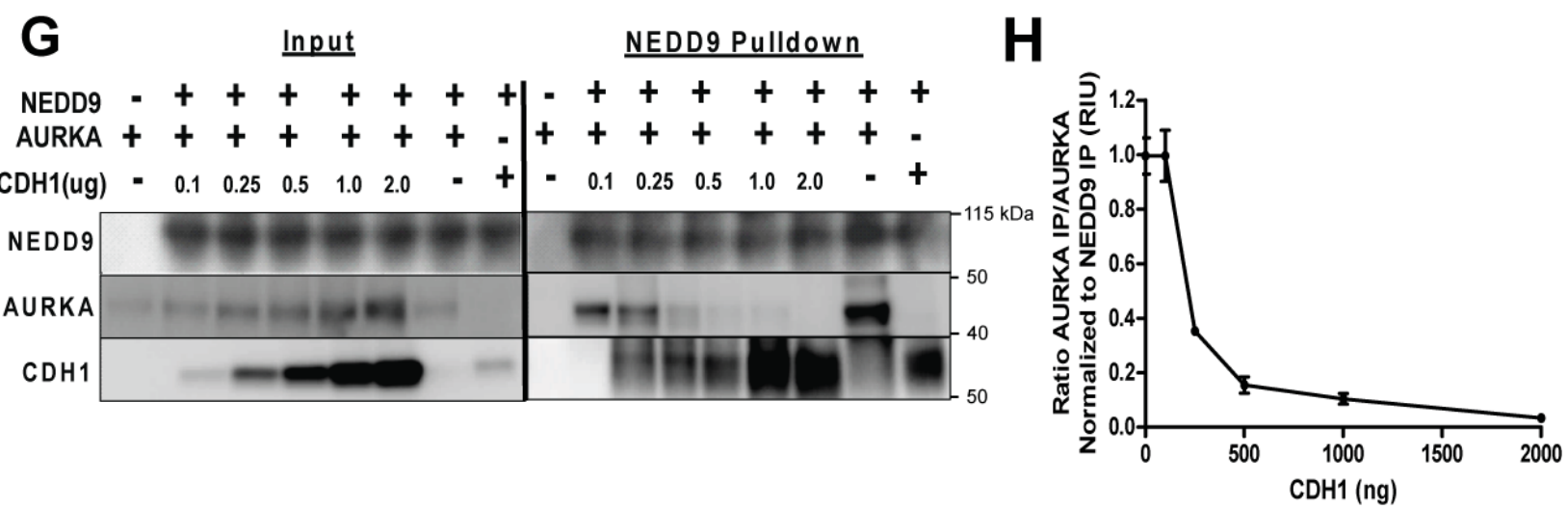

Figure 3, Ice 
A

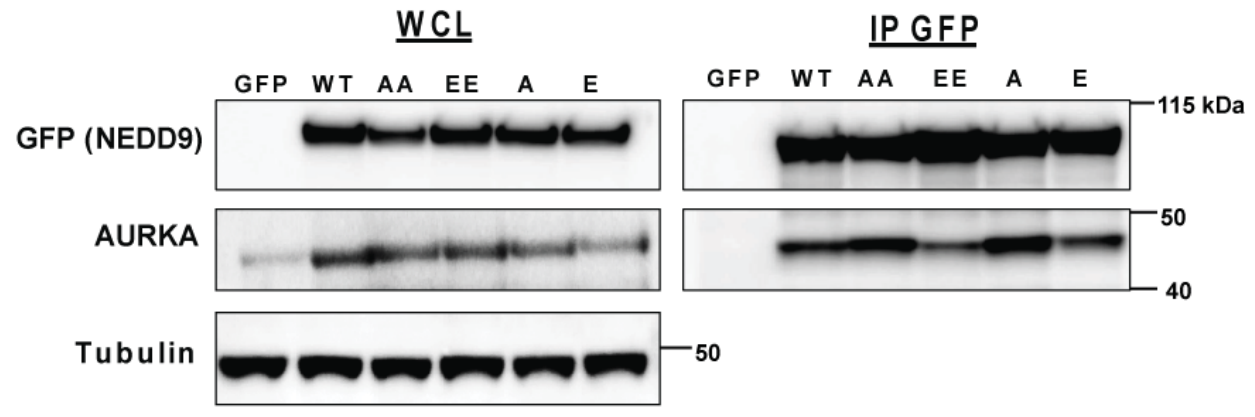

B

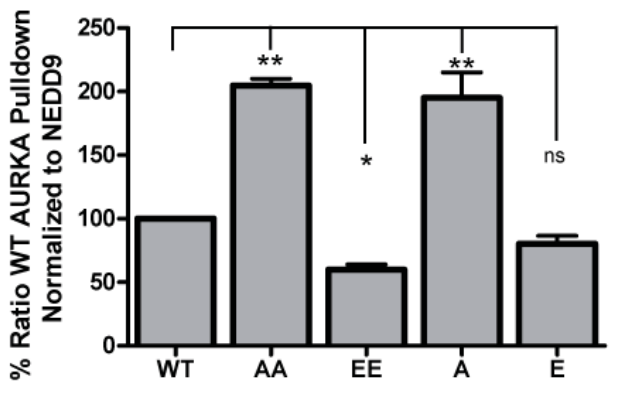

C

\begin{tabular}{|c|c|c|c|c|c|c|}
\hline \multirow[b]{2}{*}{ Dox (hrs) } & \multicolumn{2}{|c|}{ Empty } & \multicolumn{2}{|c|}{ AA } & \multicolumn{2}{|c|}{ EE } \\
\hline & 24 & 48 & 24 & 48 & 24 & 48 \\
\hline & & & & & & \\
\hline
\end{tabular}

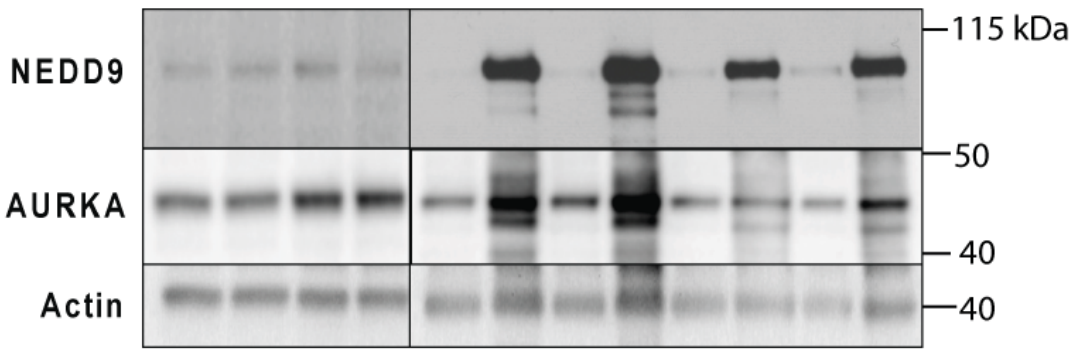

D

Figure 4, Ice

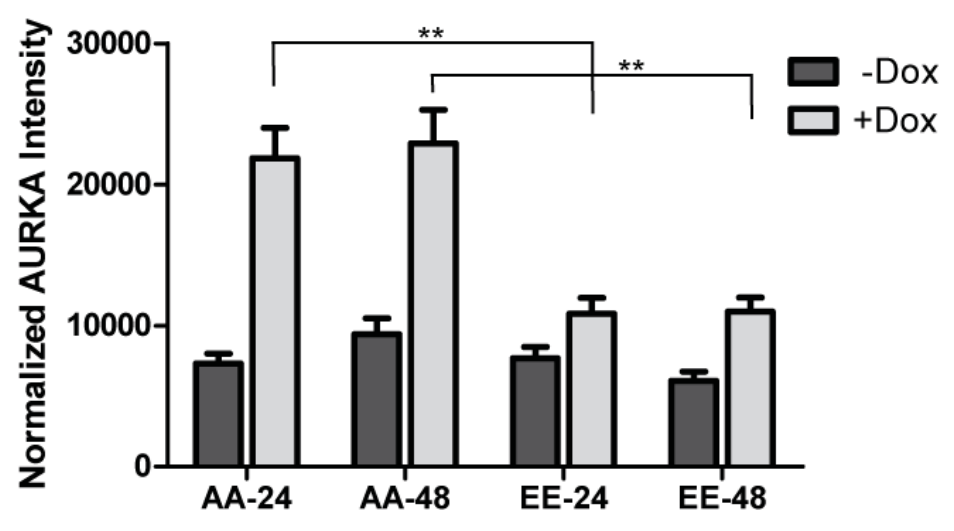


A.

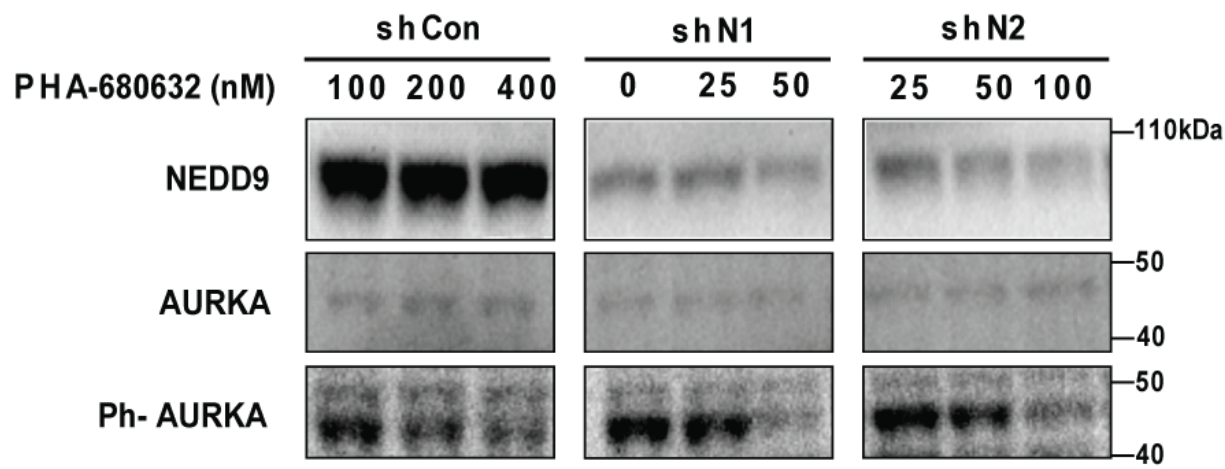

B.
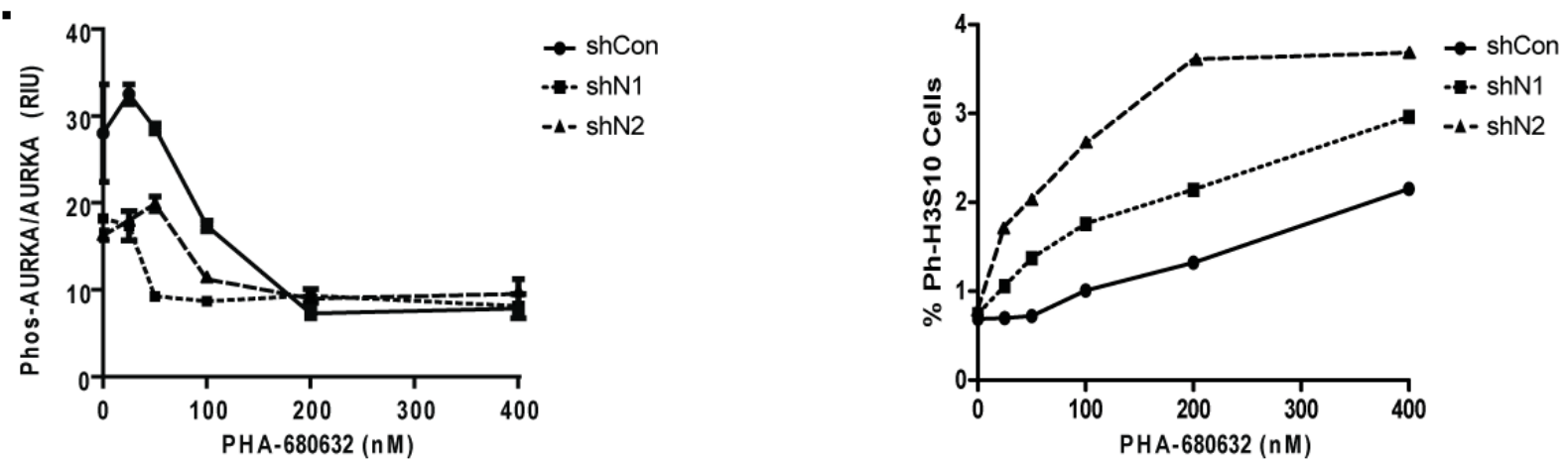

C.

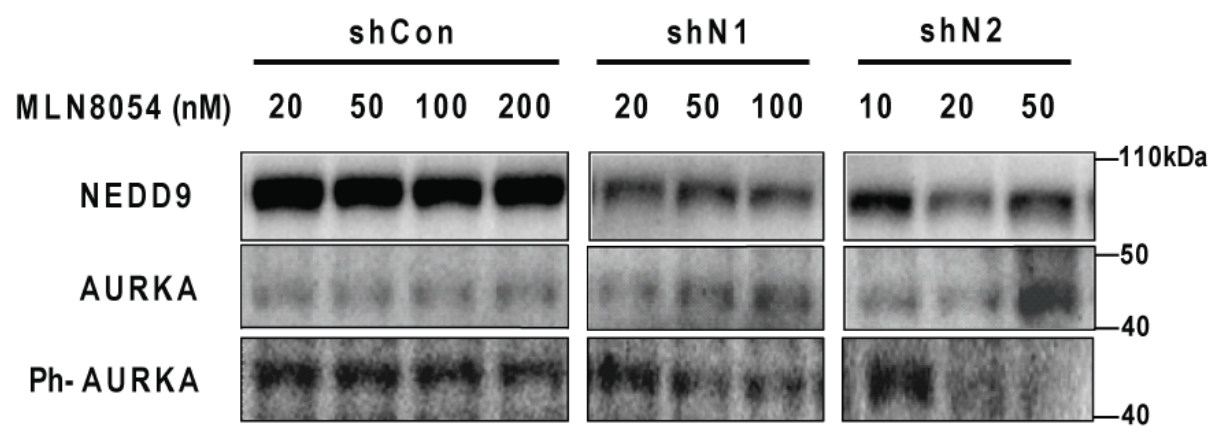

D.
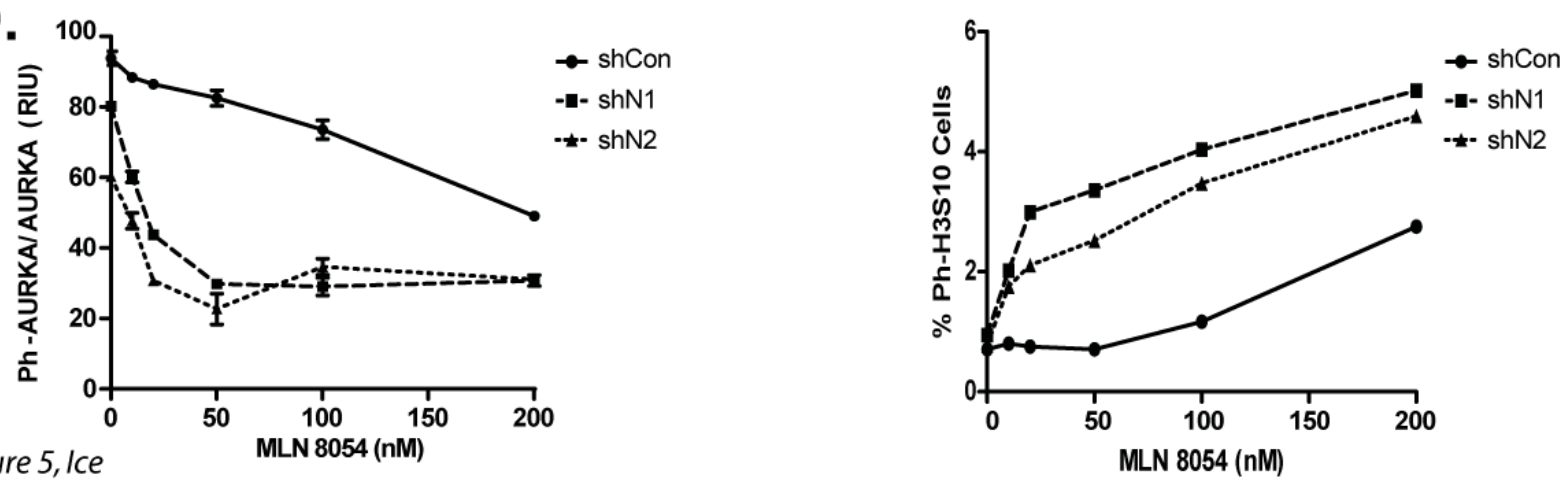
A

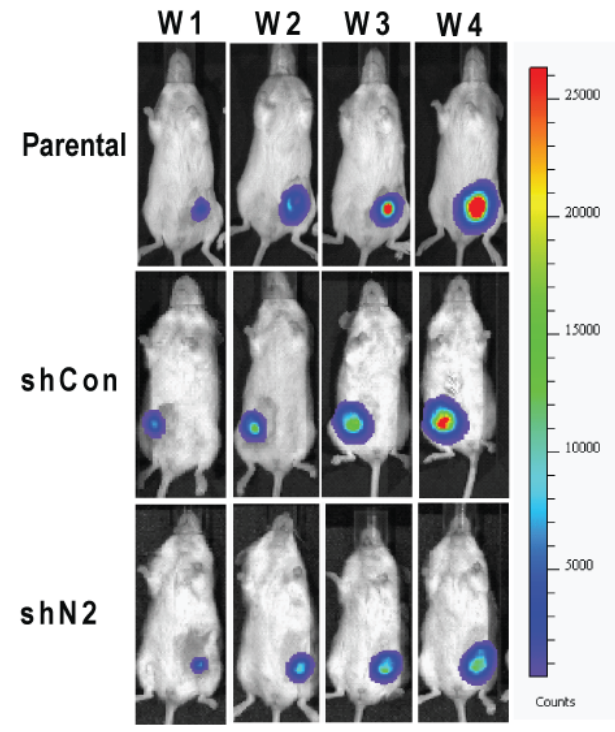

C

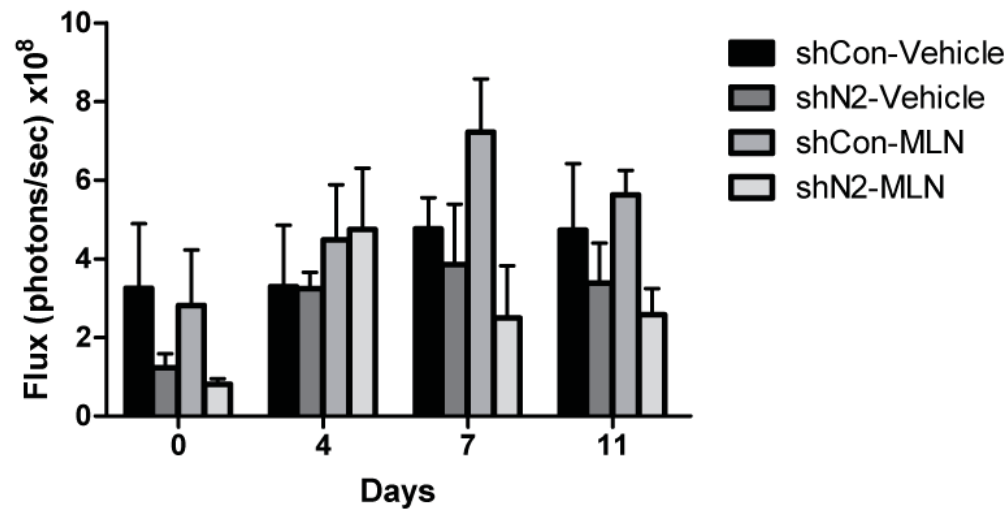

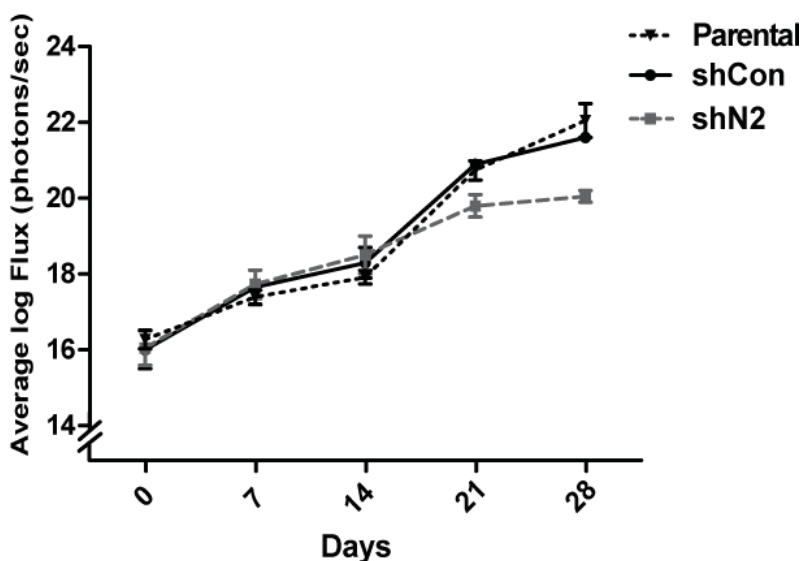

\section{E}

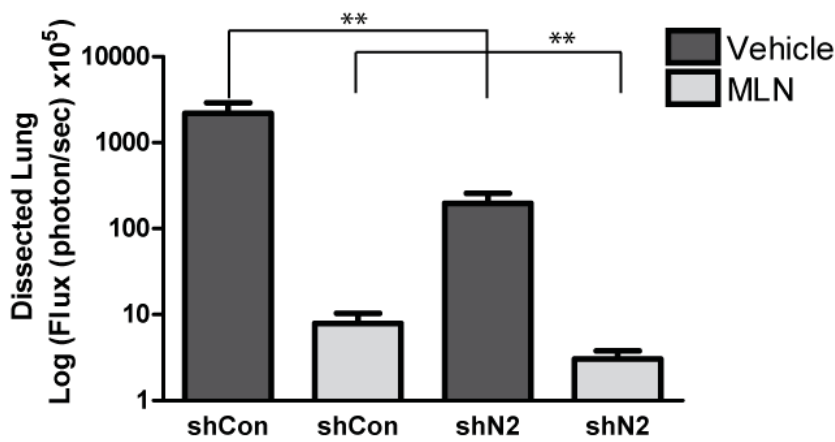

Figure 6, Ice 
A

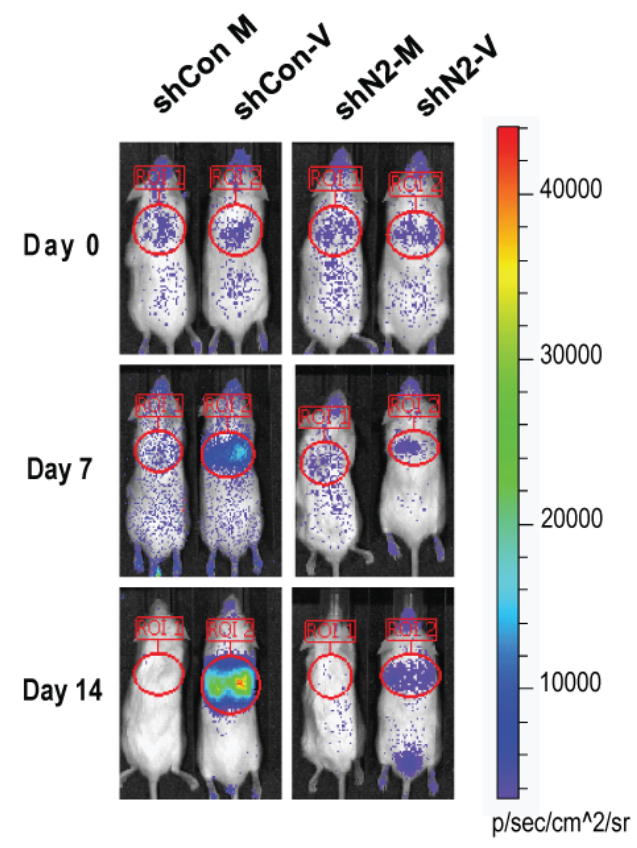

B

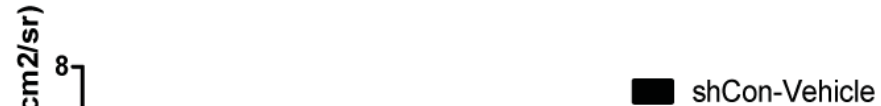

$\square$ shN2- Vehicle

$\square$ shCon-MLN

$\square$ shN2-MLN

C

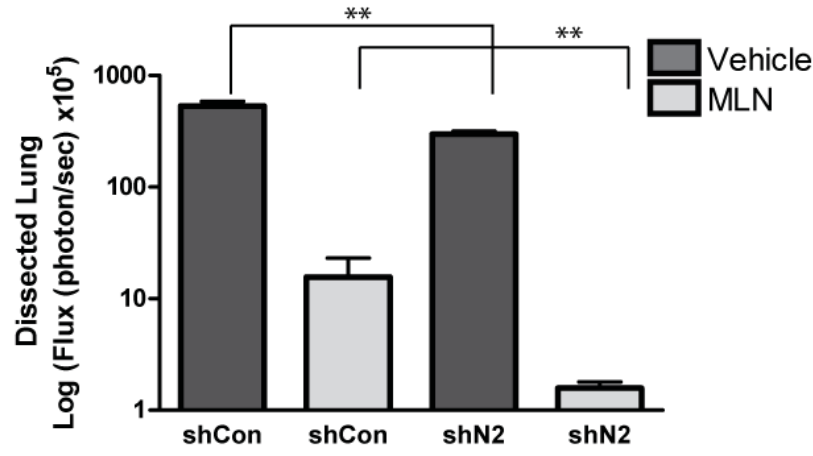

D

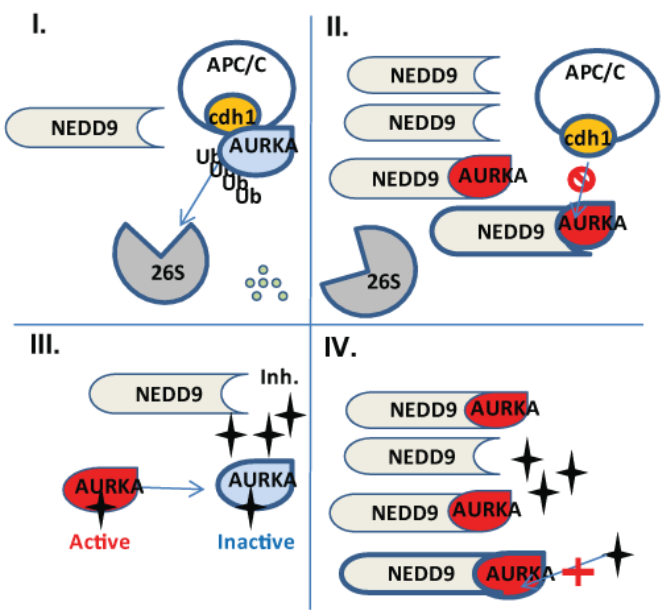

Figure 7, Ice 


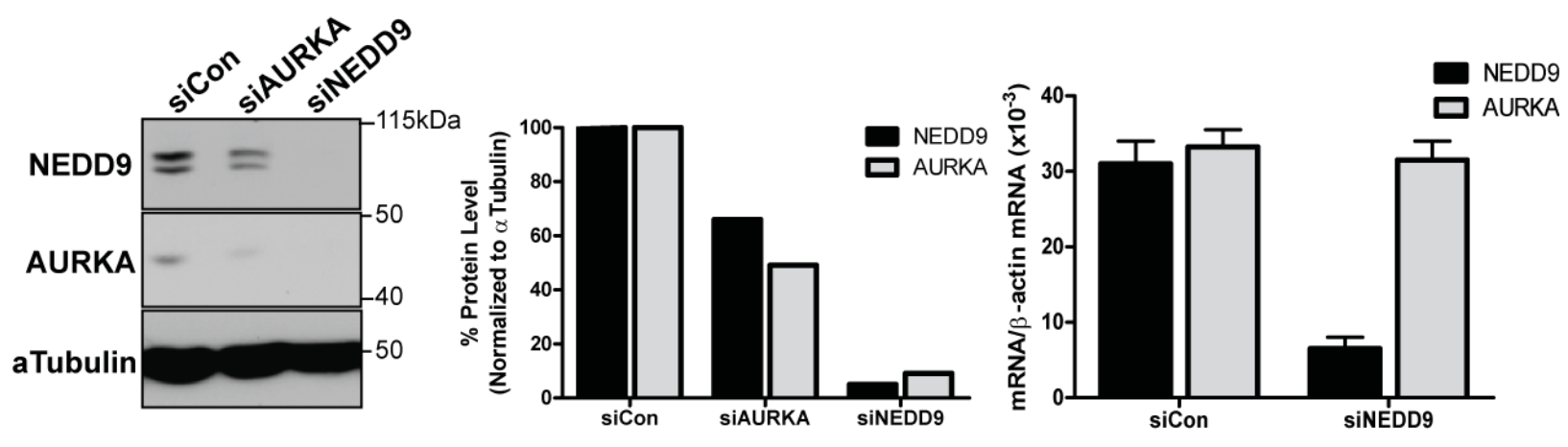

C
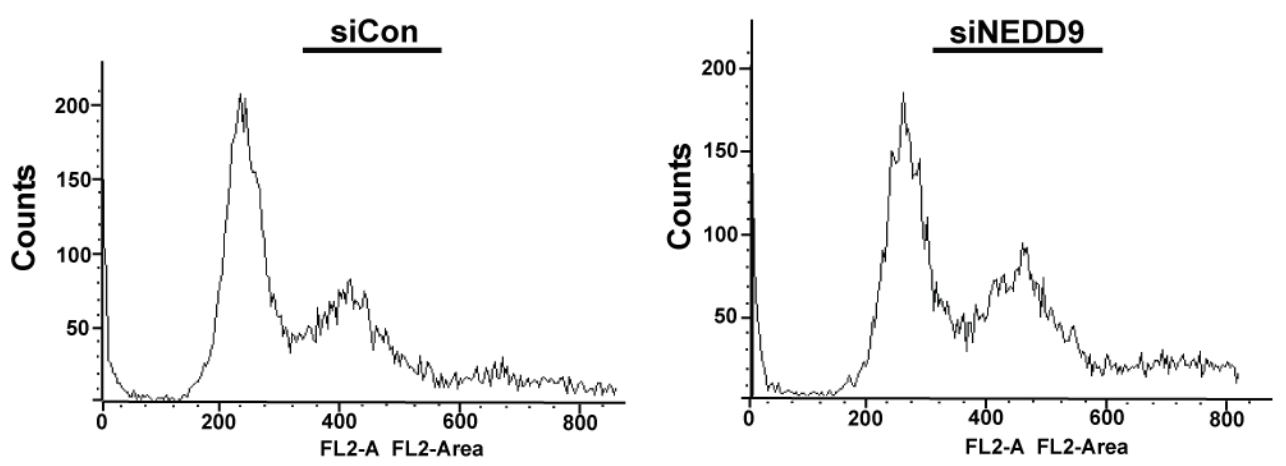

D
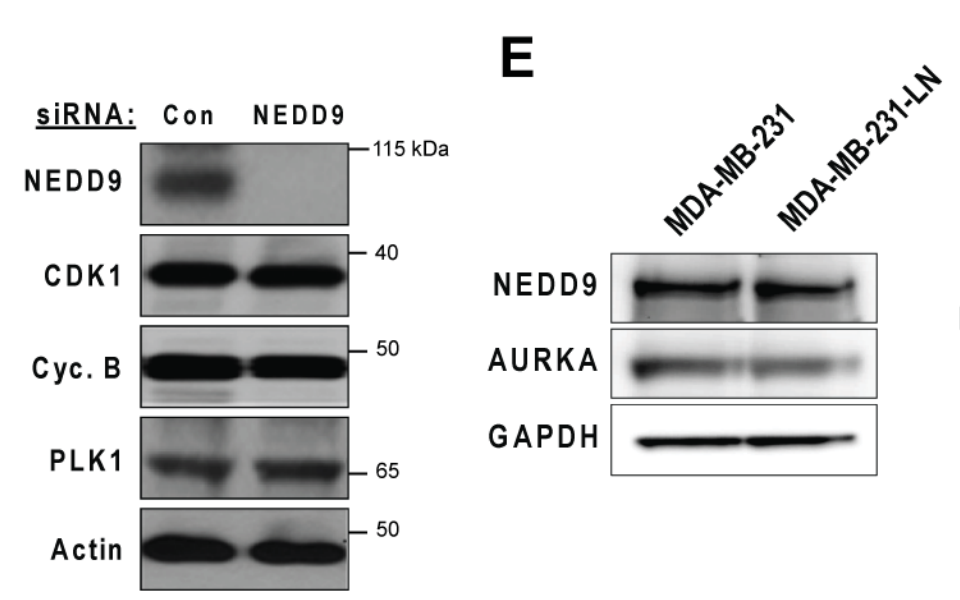

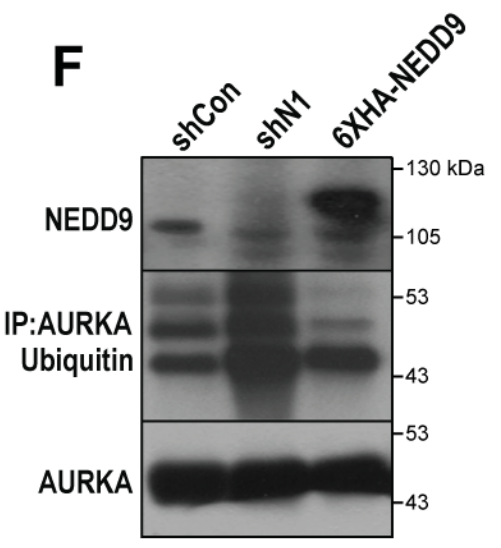

Suppl \#1, Ice 
S2

shCon

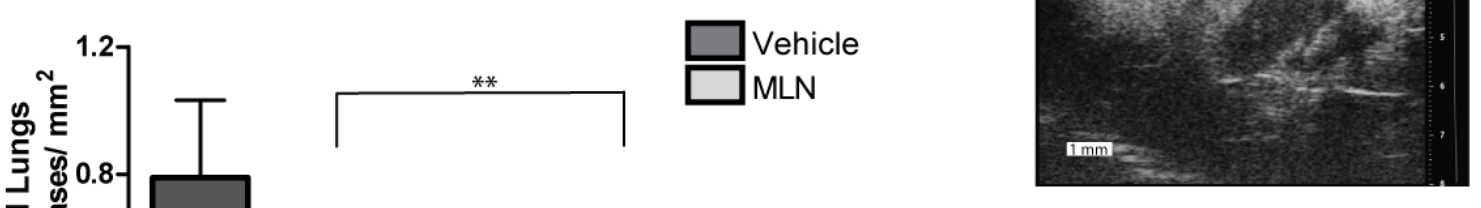

shN2

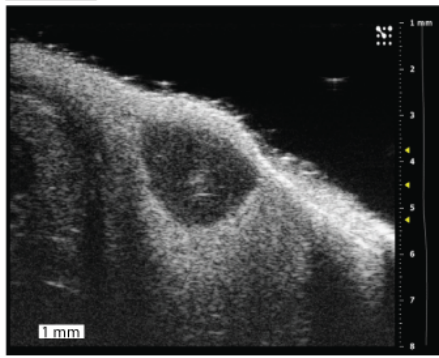

C

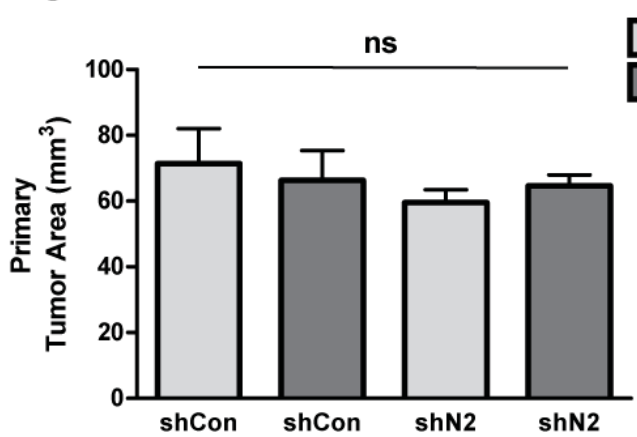

D
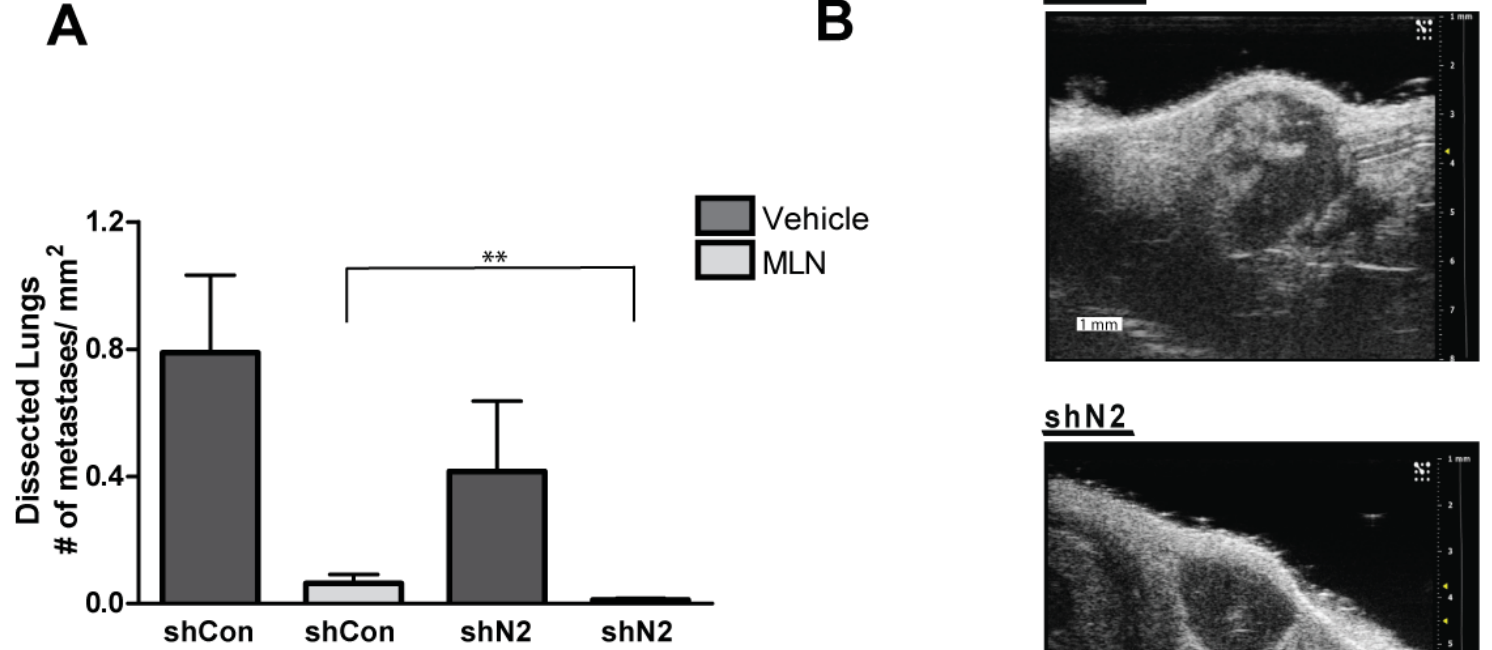

B
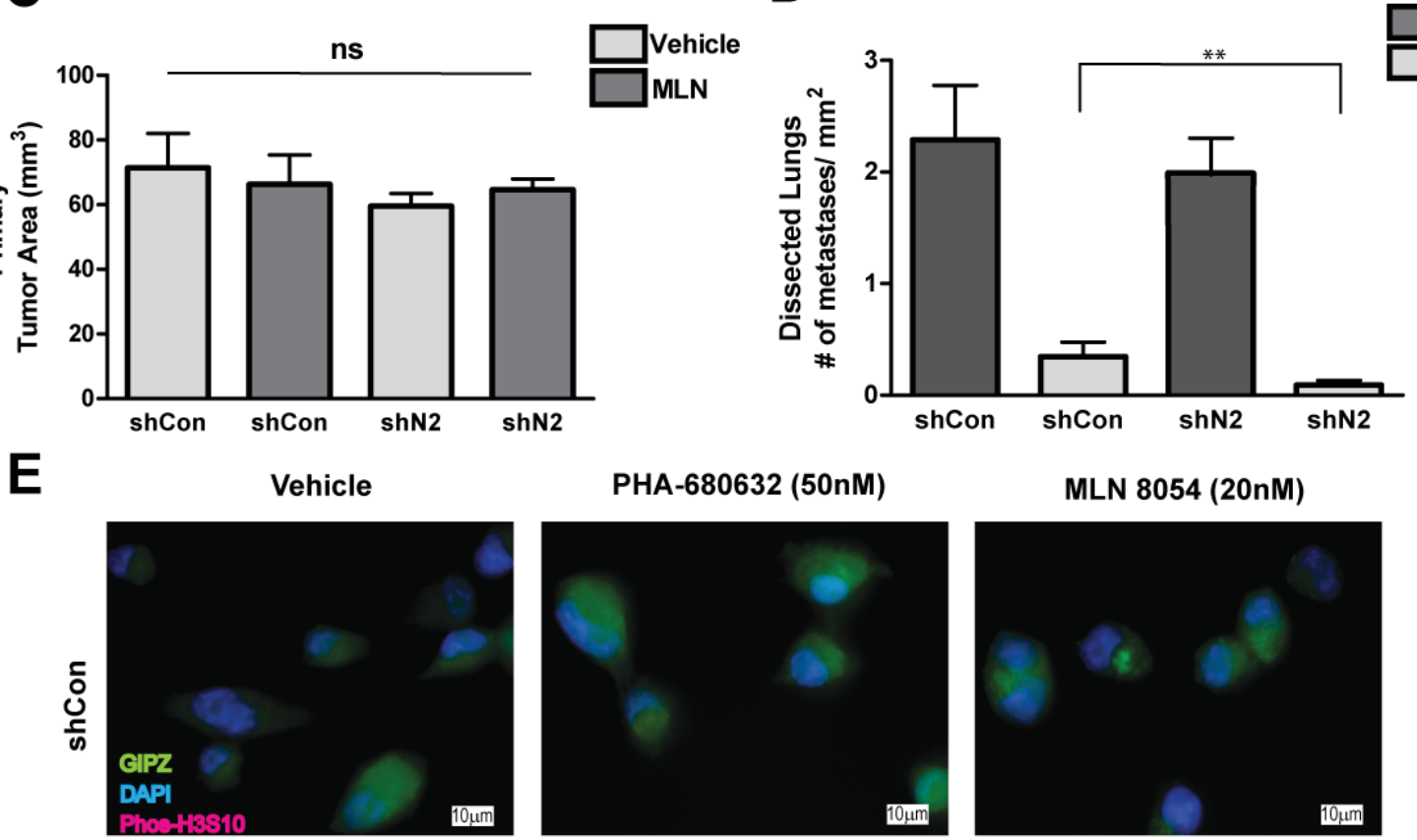

PHA-680632 (50nM)

MLN 8054 (20nM)
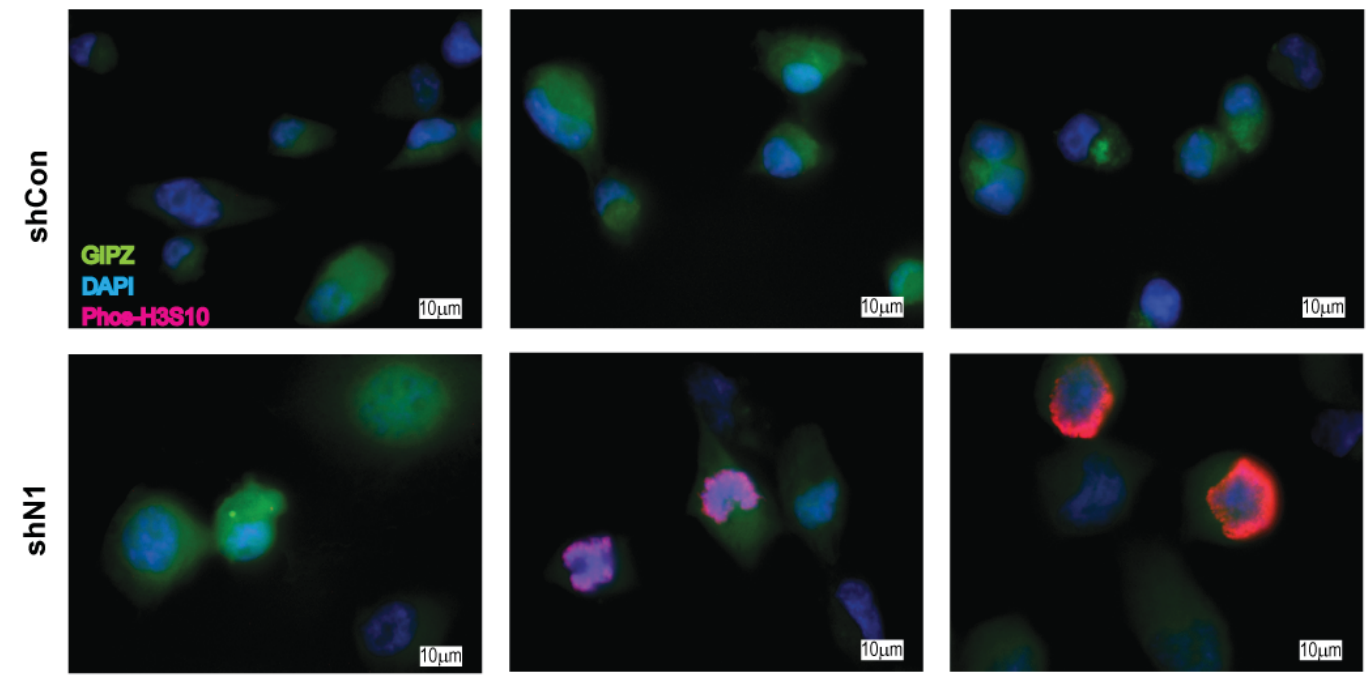

Suppl \#2, Ice 


\section{ST1.}

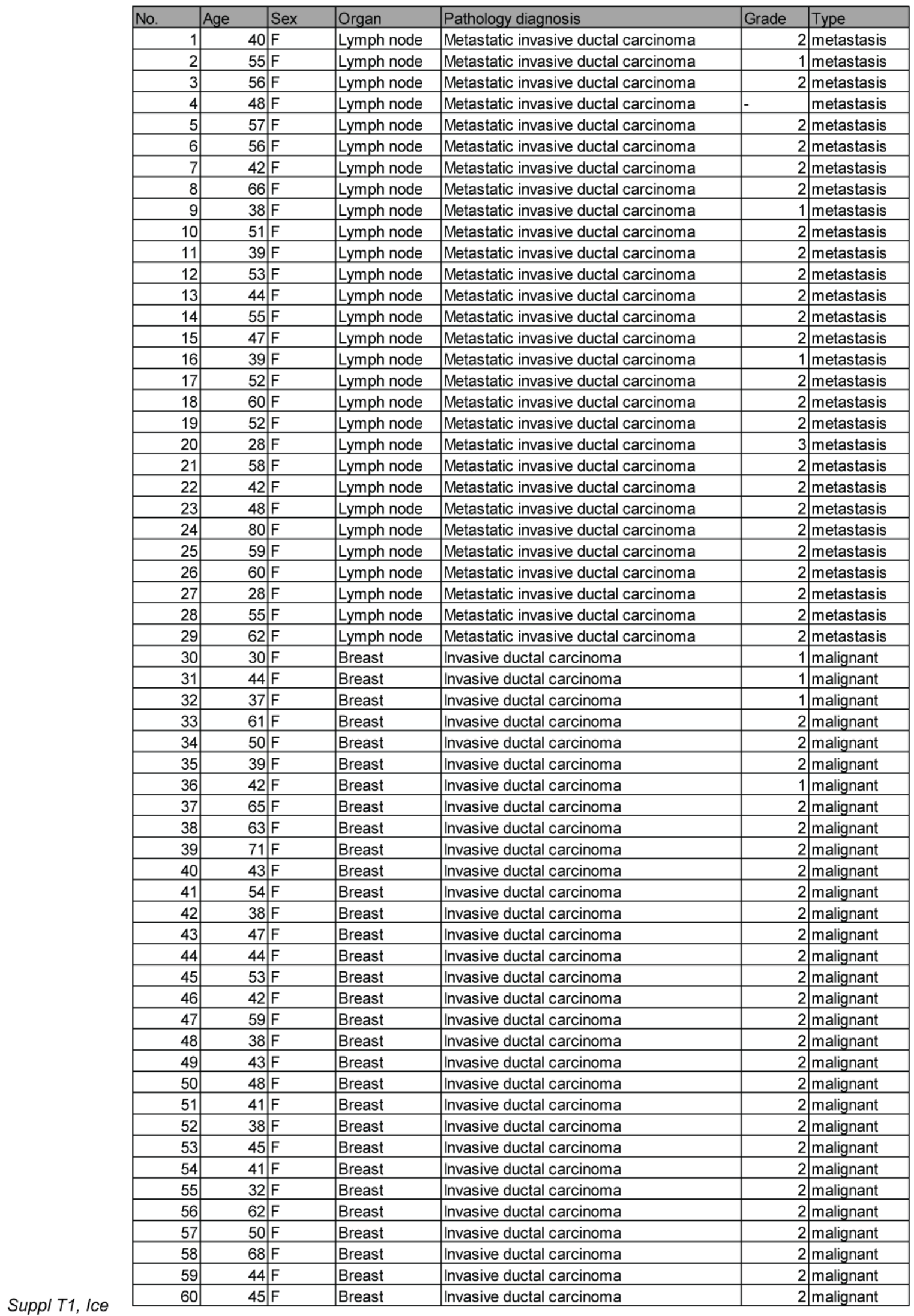


ST1. (continued)

\begin{tabular}{|c|c|c|c|c|c|c|}
\hline No. & Age & Sex & Organ & Pathology diagnosis & Grade & Type \\
\hline 60 & 45 & $F$ & Breast & Invasive ductal carcinoma & & 2 malignant \\
\hline 61 & 29 & $\mathrm{~F}$ & Breast & Invasive ductal carcinoma & & 2 malignant \\
\hline 62 & 67 & $\mathrm{~F}$ & Breast & Invasive ductal carcinoma & & 1 malignant \\
\hline 63 & 54 & $\mathrm{~F}$ & Breast & Invasive ductal carcinoma & & 2 malignant \\
\hline 64 & 35 & $\mathrm{~F}$ & Breast & Invasive ductal carcinoma & & 2 malignant \\
\hline 65 & 43 & $\mathrm{~F}$ & Breast & Invasive ductal carcinoma & - & malignant \\
\hline 66 & 29 & $\mathrm{~F}$ & Breast & Invasive ductal carcinoma & & 2 malignant \\
\hline 67 & 55 & $\mathrm{~F}$ & Breast & Invasive ductal carcinoma & & 2 malignant \\
\hline 68 & 50 & $\mathrm{~F}$ & Breast & Invasive ductal carcinoma & & 2 malignant \\
\hline 69 & 43 & $\mathrm{~F}$ & Breast & Invasive ductal carcinoma & & 2 malignant \\
\hline 70 & 43 & $\mathrm{~F}$ & Breast & Invasive ductal carcinoma & & \begin{tabular}{l|l}
2 & malignant \\
\end{tabular} \\
\hline 71 & 45 & $\mathrm{~F}$ & Breast & Invasive ductal carcinoma & & 2 malignant \\
\hline 72 & 50 & $\mathrm{~F}$ & Breast & Invasive ductal carcinoma & & 2 malignant \\
\hline 73 & 28 & $\mathrm{~F}$ & Breast & Invasive ductal carcinoma & & 2 malignant \\
\hline 74 & 55 & $F$ & Breast & Invasive ductal carcinoma & & 2 malignant \\
\hline 75 & 52 & $F$ & Breast & Invasive ductal carcinoma & & 2 malignant \\
\hline 76 & 38 & $F$ & Breast & Invasive ductal carcinoma & & 2 malignant \\
\hline 77 & 49 & $F$ & Breast & Invasive ductal carcinoma & - & malignant \\
\hline 78 & 46 & $F$ & Breast & Invasive ductal carcinoma & & 2 malignant \\
\hline 79 & 62 & $F$ & Breast & Invasive ductal carcinoma & & 2 malignant \\
\hline 80 & 42 & $F$ & Breast & Invasive ductal carcinoma & & 2 malignant \\
\hline 81 & 53 & $\mathrm{~F}$ & Breast & Invasive ductal carcinoma & & \begin{tabular}{l|l}
2 & malignant \\
\end{tabular} \\
\hline 82 & 45 & $F$ & Breast & Invasive ductal carcinoma & & 2 malignant \\
\hline 83 & 32 & $F$ & Breast & Invasive ductal carcinoma & & 2 malignant \\
\hline 84 & 29 & $F$ & Breast & Invasive ductal carcinoma & & 2 malignant \\
\hline 85 & 63 & $F$ & Breast & Invasive ductal carcinoma & & 2 malignant \\
\hline 86 & 51 & $F$ & Breast & Invasive ductal carcinoma & & 3 malignant \\
\hline 87 & 56 & $F$ & Breast & Invasive ductal carcinoma & & 2 malignant \\
\hline 88 & \begin{tabular}{l|l}
62 \\
\end{tabular} & $F$ & Breast & Invasive ductal carcinoma & & 2 malignant \\
\hline 89 & 37 & $F$ & Breast & Invasive ductal carcinoma & - & malignant \\
\hline 90 & 32 & $F$ & Breast & Invasive ductal carcinoma & & 2 malignant \\
\hline 91 & 55 & $F$ & Breast & Invasive ductal carcinoma & & 2 malignant \\
\hline 92 & 53 & $F$ & Breast & Invasive ductal carcinoma & & 2 malignant \\
\hline 93 & 49 & $F$ & Breast & Intraductal carcinoma & - & malignant \\
\hline 94 & 38 & $F$ & Breast & Intraductal carcinoma & - & malignant \\
\hline 95 & 42 & $\mathrm{~F}$ & Breast & Intraductal carcinoma & - & malignant \\
\hline 96 & 27 & $F$ & Breast & Intraductal carcinoma & - & malignant \\
\hline 97 & 41 & $F$ & Breast & Intraductal carcinoma & - & malignant \\
\hline 98 & 43 & $F$ & Breast & Intraductal carcinoma & - & malignant \\
\hline 99 & 32 & $F$ & Breast & Intraductal carcinoma & - & malignant \\
\hline 100 & 58 & $F$ & Breast & Intraductal carcinoma & - & malignant \\
\hline 101 & 67 & $F$ & Breast & Intraductal carcinoma & - & malignant \\
\hline 102 & 49 & $\mathrm{~F}$ & Breast & Intraductal carcinoma & - & malignant \\
\hline 103 & 32 & $F$ & Breast & Intraductal carcinoma & - & malignant \\
\hline 104 & 81 & $F$ & Breast & Intraductal carcinoma & - & malignant \\
\hline 105 & 43 & $F$ & Breast & Intraductal carcinoma & - & malignant \\
\hline 106 & 46 & $F$ & Breast & Intraductal carcinoma & - & malignant \\
\hline 107 & 60 & $\mathrm{~F}$ & Breast & Intraductal carcinoma & - & malignant \\
\hline 108 & 64 & $F$ & Breast & Intraductal carcinoma & - & malignant \\
\hline 109 & 40 & $F$ & Breast & Intraductal carcinoma & - & malignant \\
\hline 110 & 31 & $F$ & Breast & Normal breast tissue & - & normal \\
\hline 111 & 45 & $F$ & Breast & Normal breast tissue & - & normal \\
\hline 112 & 53 & $F$ & Breast & Normal breast tissue & - & normal \\
\hline 113 & 36 & $F$ & Breast & Normal breast tissue & - & normal \\
\hline 114 & 35 & $F$ & Breast & Normal breast tissue & - & normal \\
\hline 115 & 44 & $F$ & Breast & Normal breast tissue & - & normal \\
\hline 116 & 33 & $F$ & Breast & Normal breast tissue & - & normal \\
\hline 117 & 46 & $F$ & Breast & Normal breast tissue & - & normal \\
\hline 118 & 15 & $F$ & Breast & Normal breast tissue & - & normal \\
\hline 119 & 35 & $F$ & Breast & Normal breast tissue & - & normal \\
\hline 120 & $27 \mid$ & $F$ & Breast & Normal breast tissue & - & normal \\
\hline
\end{tabular}




\section{Study 2: Depletion of NEDD9 leads to inactivation of MMP14 by TIMP2 and prevents invasion and metastasis of breast cancer cells.}

Sarah L. McLaughlin ${ }^{5^{*}}$, Ryan J. Ice ${ }^{5^{*}}$, Anuradha Rajulapati ${ }^{5}$, Polina Y. Kozyulina ${ }^{1}$, Ryan H. Livengood $^{4}$, Varvara K. Kozyreva ${ }^{5}$, Yuriy V. Loskutov ${ }^{5}$, Mark V. Culp ${ }^{3}$, Scott A. Weed ${ }^{2,5}$, Alexey V. Ivanov ${ }^{1,5}$ and Elena N. Pugacheva ${ }^{1,5 \# .}$

${ }^{1}$ Department of Biochemistry, ${ }^{2}$ Department of Neurobiology and Anatomy, ${ }^{3}$ Department of Statistics, ${ }^{4}$ Department of Pathology, ${ }^{5}$ Mary Babb Randolph Cancer Center, West Virginia University School of Medicine, Morgantown, WV, 26506

S.L. McLaughlin* and R. J. Ice* contributed equally to this work.

Published in Mol Cancer Res. 2013 Nov 7.

\#Corresponding author:

Elena N. Pugacheva

Department of Biochemistry and Mary Babb Randolph Cancer Center, PO Box 9142,

1 Medical Center Drive,

West Virginia University School of Medicine,

Morgantown, WV, 26506.

Phone: (304) 293-5295;

Fax: (304) 293-4667;

Email: epugacheva@hsc.wvu.edu.

Keywords: NEDD9, invasion, metastasis, breast cancer, and MMP14 


\begin{abstract}
The scaffolding protein NEDD9 is an established pro-metastatic marker in several cancers. Nevertheless, the molecular mechanisms of NEDD9 driven metastasis in cancers remain ill defined. Here, using a comprehensive breast cancer (BCa) tissue microarray, it was show that increased levels of NEDD9 protein significantly correlated with the transition from carcinoma in situ to invasive carcinoma. Similarly, it was shown that NEDD9 overexpression is a hallmark of highly invasive BCa cells. Moreover, NEDD9 expression is crucial for the protease-dependent mesenchymal invasion of cancer cells at the primary site but not at the metastatic site.

Depletion of NEDD9 is sufficient to suppress invasion of tumor cells in vitro and in vivo, leading to decreased circulating tumor cells (CTCs) and lung metastases in xenograft models.

Mechanistically, NEDD9 localized to invasive pseudopods and was required for local matrix degradation. Depletion of NEDD9 impaired invasion of cancer cells through inactivation of membrane-bound matrix metalloproteinase MMP14 by excess TIMP2 on the cell surface. Inactivation of MMP14 is accompanied by reduced collagenolytic activity of soluble metalloproteinases MMP2 and MMP9. Re-expression of NEDD9 is sufficient to restore the activity of MMP14 and the invasive properties of BCa cells in vitro and in vivo. Collectively, these findings uncover critical steps in NEDD9-dependent invasion of BCa cells. Implications: This study provides a mechanistic basis for potential therapeutic interventions to prevent metastasis.
\end{abstract}




\section{Introduction}

The cytoplasmic docking protein, NEDD9 has emerged as a biomarker of melanoma, pancreatic, and lung cancer metastasis and is required for the migration of tumor cells (1-6). The overexpression of NEDD9 leads to mesenchymal protease-dependent migration $(4,7)$. NEDD9 promotes epithelial-mesenchymal transition (EMT) and invasion of BCa cells (8).

Extracellular matrix (ECM) proteolysis is dependent upon soluble and membrane bound matrix metalloproteinases (MMPs) (9). Membrane bound MMP14 is a key enzyme involved in ECM degradation and invasion of tumor cells. The activity of MMP14 is regulated by phosphorylation, proteolysis, and endocytosis (10-11). MMP14 is activated by furin and is inhibited by tissue inhibitor of MMP, TIMP2, which makes a stable complex with the activated MMP14 (12-13). The tertiary complex of MMP14, TIMP2, and proMMP2 is required to form on the cell surface to activate MMP2 by a neighboring MMP14 free of TIMP2 (14). The presence of excess TIMP2 results in inhibition of MMP14 activity and decreased processing of proMMP2 to active MMP2 (15). In invasive cancer cells, MMP14 is localized at the membrane of invadopodia, ventral actin-based membrane protrusions (16-17). Local ECM degradation is initiated upon the assembly of: F-actin, cortactin, N-WASP, and the Arp2/3 complex (17-18). Functional mature invadopodia form when F-actin and cortactin-rich puncta initiate the accumulation of MMP14, thus forming proteolytically active protrusions (16). Activity of MMP14 is required for invasion of 3D collagen matrices in tissue culture and in nude mice (19-20).

While NEDD9 deficient cells are characterized by reduced migration accompanied with reduced levels of Src and FAK kinase activity (21-22) the role of NEDD9 in the function of invadopodia in BCa has been unclear. Although NEDD9 and another Cas family member, p130Cas, were previously identified in invadopodia of cancer cells (23-25), the differences between NEDD9 and p130Cas function in invasion are currently unknown.

In transgenic oncogene driven mammary tumor models such as MMTV-PyVmT or MMTV-Her2/Erb2 genetic ablation of NEDD9 leads to delays in tumor initiation and growth but has a limited effect on metastasis (21-22). The tumors arising in MMTV-Her2/NEDD9-/- or PyVmT/NEDD9-/- mice underwent a dramatic mutational selection that overcame the initial requirement for NEDD9. In this context it is difficult to determine the requirement for NEDD9 in metastasis since the selection of alternative signaling pathways happened prior to tumor formation. Also, it is currently unknown what stages of metastasis are affected by NEDD9.

Overexpression of NEDD9 is often mentioned in diverse cancers (1-4). However, as of now, a comprehensive analysis of NEDD9 expression with respect to BCa progression has not been conducted; To address these questions, we analyzed NEDD9 protein expression in a 
tissue microarray (TMA) consisting of 200 progressive BCa patient biopsies and in a panel of diverse BCa cell lines. We found that NEDD9 is overexpressed in invasive breast ductal carcinomas and invasive cell lines. We discovered that NEDD9 is involved in regulation of MMP14 activity on the cell surface, but not expression. Depletion of NEDD9 leads to decrease in proteolytic activity of soluble MMPs and transmembrane MMP14, resulting in the ablation of cancer cell invasion in vitro and in vivo. The decrease in activity was due to the association of MMP14 with its inhibitor TIMP2 on cell surface. Depletion of TIMP2 or MMP14 expression or addition of excess of recombinant TIMP2 to control cells led to a similar phenotype, suggesting that excessive TIMP2 bound to the MMP14 potentially is the primary reason for decreased invasion in carcinoma cells upon depletion of NEDD9.

Using inducible shRNAs against NEDD9 in xenograft models, we dissected particular stages of metastasis dependent upon NEDD9 expression and thus define the sensitive stages of tumor progression where anti-NEDD9 therapy could be applied to prevent metastasis. We found that reduction of NEDD9 expression in established tumors leads to a drastic decrease in MMPs activity and number of circulating tumor cells resulting in a decrease in the overall number and size of pulmonary metastases. Collectively, our findings suggest a novel mechanism for NEDD9 in accelerating cell invasion through regulation of MMP14 by TIMP2 thus defining the new therapeutic approach for anti-metastatic strategies via manipulation of NEDD9 expression. 


\section{Materials and methods}

Plasmids and cell culture. Cell lines MDA-MB-231, MDA-MB-453, ZR-75-1, BT-549, MCF10A, MCF7, AU-565, BT-20, were purchased from and authenticated by American Type Culture Collection (ATCC), MDA-231-LN (Caliper Life Sci.) and grown based on manufacturer's recommendations. shRNA expressing constructs against NEDD9, control (sequences available upon request) and smart pool siRNAs against MMP14 and TIMP2 and siControl were purchased from ThermoFisher Scientific as ready to use siRNAs or in pGIPZ, or in doxycyclineinducible pTRIPZ vectors. Lentiviral particles were prepared as previously described (26). For rescue experiments wild type cDNA of mouse NEDD9 was subcloned into pLUTZ lentiviral vector under doxycycline-inducible promoter (27). Cell medium and supplements were purchased from ATCC (Sigma).

MMP Antibody Array. MMP Antibody Arrays were purchased from RayBiotech, Inc. and assays were carried out according to the manufacturer's protocol using whole cell lysate (WCL) and conditioned for $24 \mathrm{~h}$ serum free medium (SFM).

Fluorescent-gelatin degradation assay. Foci of degraded matrix were visible as dark areas that lack fluorescence in the FITC-gelatin matrix (Life Technologies). Cells with pseudopods were identified by the presence of at least one actin/cortactin aggregate within the cell. Degradation per cell area was analyzed using ImageJ (NIH) as described (16). At least 100 cells were counted per each experimental condition. Data were pooled from multiple independent experiments.

DQ Collagen Assay. Cells expressing doxycycline-inducible shRNAs against NEDD9 and red fluorescent protein (RFP) embedded in DQcollagen I/IV combined with matrigel (BD Biosciences) to allow for cell tracing and dose-dependent manipulation of NEDD9 depletion. shRNA expression was induced for $72 \mathrm{~h}$. DQ collagen I/IV/matrigel assays were carried out according to a previously published protocol (28). Detailed protocol for data acquisition and analysis outlined in supplementary material.

EnzChek Gellatinase/Collagenese assay. Assay was purchased from Life Technologies and performed accordingly to manufacturer's recommendations using shNEDD9 and shCon conditioned medium (24h). Fluorescence was measured at 485/545mn. 
Gelatin zymography. The gelatin zymography protocol was performed as previously described $(16,24)$. Briefly, $4 \mathrm{ml}$ of $24 \mathrm{~h}$ conditioned media was collected from cells, concentrated using AmiconUltra-4 columns and 1/20 of original volume loaded on the Zymogram Gelatin Gels (Life Technologies) and developed according to the manufacturer's recommendations.

MMP14-specific fluorogenic substrate degradation assay. $2 \times 104$ cells were plated on 96w plate, for 16 hours, followed by 1 hour in L15 media (Life Technologies). MMP14-specific fluorogenic substrate (Millipore) was introduced in final concentration $10 \mu \mathrm{M}$. Fluorescence was measured at 328/400mn every 5 minutes for 12 hours, using Synergy H4 Plate Reader (BioTek). Averaged background signal was subtracted, and data plotted curves described by equation for one site binding kinetics and fitted to each data set with the R2 of 0.8 The curves were significantly different between control and NEDD9 deficient cells according to the Extra sum ofsquares $F$ test, $p<0.0001$.

ECM coated Boyden chamber invasion assays. Invasion assays were performed using BD FluoroBlok ${ }^{\mathrm{TM}}$ insets coated with matrigel (BD Biosciences). Cells were added to the top chamber in serum free media, 10\%FBS supplemented MEM was added to the bottom chamber, and incubated for $8 \mathrm{~h}$. Once cells migrated through the matrix, they were labeled with Calcein AM (Life Technologies) and detected by fluorescence plate reader (Genios) at 485/530nm excitation/emission.

Cell viability Trypan Blue exclusion assay. MDA-MB-231-shCon, -shNEDD9 cells were cultured in suspension using Ultra-Low attachment plates (Fisher Scientific) for 36 hours; 0.1 $\mathrm{mL}$ of a $0.4 \%$ solution of trypan blue in PBS was added to $1 \times 106 / \mathrm{mL}$ of cells. The total number of cells and the number of blue staining cells were calculated by Countess automated cell counter (Life Technologies). Number of viable cells $\times 104 \times 1.1=$ cells $/ \mathrm{mL}$ culture.

Fluorescence Activated Cell Sorting (FACS). 1x106 cells, with or without permeabilization by 0.5\% Tween20 (Sigma), were stained for MMP14 (Novus International). Secondary fluorescence conjugated antibodies (BD Biosciences) were used at a concentration 0.5ug/1 x106 cells. Cells were pre-blocked with $5 \mathrm{mg} / \mathrm{ml}$ of Human IgG (Sigma). As a control, nonspecific primary antibody, either Rabbit or mouse lgG were used (Sigma). 
Western blotting (WB). Western blotting was done using standard procedures $(26,30)$. Primary antibodies included mouse anti-NEDD9 mAb (2G9) (29), anti-phTyr, (Cell Signaling Technology Inc.), anti-p130Cas, -MMP2, -MMP9, -GAPDH, -RFP (ThermoFisher), anticortactin, -MMP14 (Novus), anti- $\beta$-actin, - $\alpha$-tubulin, (Sigma) antibodies. Secondary HPRconjugated antibodies and chemiluminescence-based detection and quantification methods were previously described (30).Recombinant

TIMP2 titration assay. Recombinant human TIMP2 was purchased from Life Technologies, diluted in cell culture medium and added to the cells in $0-100 \mathrm{nM}$ final concentration for 6-12h. After indicated times cells number of invaded cells was calculated as described in Boyden chamber invasion assay.

Immunofluorescence (IF). IF was performed as previously described (30). Primary antibodies included: rabbit anti-phTyr, -Arp3, -N-WASP (Cell Signaling Technology), anti-cortactin, MMP14 (Novus), anti-cortactin (4F11). The secondary antibodies included: Alexa Fluor 405, 488, 555 donkey anti-rabbit, and Alexa Fluor 647 goat anti-mouse and Rhodamine-540 or PacificBlue-405-conjugated phalloidin (Life Technologies). Images were captured by confocal microscope LSM 510 (Carl Zeiss). To analyze IF data, volumes of positively stained structures were evaluated per cell. Quantifications were performed on z stack projections, and collected using standard acquisition parameters with Image $(\mathrm{NIH})$ and LSM Image analysis software (Carl Zeiss).

Tissue Microarrays (TMA) and Immunohistochemistry (IHC). The high density BCa tissue microarrays were purchased from US Biomax Inc., and content is outlined in Supplementary Table 1. All procedures were approved by the Institutional Biohazard Committee of West Virginia University. TMA sections were deparaffinized, endogenous peroxidase activity was quenched with $3 \%$ hydrogen peroxide, and slides were incubated with anti-NEDD9 antibodies ( $1: 100$ dilution) overnight at 4C; followed by staining with secondary peroxidase-labeled antibodies and developed with DAB (DAKO). Positive and negative controls were included with each run. Staining was done in duplicate. The IHC analysis and scoring protocol is available in Supplementary materials.

Lung metastasis analysis. Lungs were collected and fixed in 4\%PFA for 24-48h embedded in paraffin by WVU Tissue Bank as previously described (30). Serial 6-10 $\mu \mathrm{m}$ think sections were 
cut at each $40 \mu \mathrm{m}$ steps through the whole organ. Sections were subjected to hematoxylin and eosin (H\&E) staining. Metastatic lesions were visually identified and images were acquired using Zeiss Axioplan microscope with x4 and x10 NA1.6 objective, followed by higher x100 magnification evaluation of whole section. Multiple sections from each lung/mouse were analyzed in each cohort. Quantification of the metastases was done by WVU pathologist (Dr. Livengood) using serial sections of lungs and H\&E staining. Results are plotted as mean number of metastases per mm2 +/ S.E.M, 3 independent experiments, $n=6-10$ per treatment group.

Animal Studies. NOD.Cg-Prkdcscid I/2rgtm1Wj//SzJ/(NSG) immunodeficient mice were purchased from the Jackson Laboratory. Animals were housed in the WVU Animal Facility under pathogen-free conditions and approved by the Institutional Animal Care and Use Committee protocol. Primary tumors and lungs were collected, processed for histopathology, and analyzed by the WVU Tissue Bank Core Facility.

\section{Animal Bioluminescence Imaging (BLI) mammary fat pad injections. For orthotopic} injections luciferase-expressing MDA-MB-231LN and BT-549 cells expressing Control (Con) or NEDD9 (N1, N4) targeting shRNAs were grown, trypsinized, and resuspended in PBS (1×107 cells $/ \mathrm{ml}$ ), and $0.1 \mathrm{ml}$ was injected into the 4th inguinal mammary gland of 6-8 weeks old NSG female mice. Tumor cells were injected in 6 mice and the experiments were repeated at least 3 times resulting in a total of 18 mice per cell line/per shRNA. Mice were imaged weekly for quantitative evaluation of tumor growth and dissemination for up to 6 weeks. For doxycyclineinducible shRNAs Dox diet (BioServ, S3888, 200mg/kg doxycycline) was introduced to start the shRNA expression when primary tumors in all experimental cohorts had reached similar size of $200 \mathrm{~mm} 3$ (2-3 weeks post injection). shRNA expression was monitors by the increase in RFP fluorescence. $150 \mathrm{mg} / \mathrm{kg}$ D-Luciferin (Perkin Elmer) was injected into the peritoneum. Images were obtained using the IVIS® Lumina-II Imaging System and Living Image-4.0 software (Perkin Elmer).

\section{Isolation and characterization of circulating tumor cells (CTC) from Peripheral Blood.}

Blood was collected from animals weekly via submandibular bleeding. About $200 \mu$ l of blood was collected into centrifuge tubes containing anticoagulants (EDTA, heparin). Peripheral blood was depleted for erythrocytes using RBC Lysis buffer (eBiosciences) according to manufacturer's guidelines. The presence of nucleated tumor cells was detected by co-staining with DAPI and 
quantified by direct fluorescence, of RFP-induced by Doxycycline only in tumor cells (red) was measured under fluorescence microscope.

Statistical Analysis. Statistical comparisons were made using two-tailed Student's t test. When more than two groups were analyzed, one-way analysis of variance (ANOVA) was used. $P \leq 0.05$ was considered to be significant. Experimental values were reported as the means with +/S.E.M. All calculations were made using the GraphPad InStat software. Random forests analysis provides an aggressive machine learning approach for classifying data into groups using ensembles of regression trees. Using this approach, we quantified the influence of NEDD9 IHC intensity staining in prediction of disease stage by the construction of the regression trees using a variable importance score averaged over all the trees. 


\section{Results}

Increase in NEDD9 protein correlates with an invasive state in $\mathrm{BCa}$. To assess the role of NEDD9 in cancer progression, we screened two independent TMAs using immunohistochemical staining (IHC) comprised of 200 BCa cases. Figure 1A shows representative images of NEDD9 staining in normal, DCIS (ductal carcinoma in situ), IDC (invasive ductal carcinoma), and MIDCLN (metastatic invasive ductal carcinoma, lymph node). Statistical analysis of anti- NEDD9 staining intensity performed either by automated image capture system (Fig.1B) or visual evaluation by pathologist (Suppl.Fig.1A) suggests that NEDD9 levels positively correlate with disease progression. The lowest intensity was found in normal tissue, followed by a 10 fold increase in DCIS and a 30 fold increase in IDC, whereas only a 10 fold increase in expression was observed in MIDC-LN (Fig.1B). Random forest statistical analysis of NEDD9 staining, which achieved an out-of-bag error rate of 0.49 , indicates that by using NEDD9 protein levels, one can double the predictive power over chance of classifying tumor. The independent prognostic value of NEDD9 expression had $70 \%$ probability of identifying cases of IDC (Fig.1C).

NEDD9 expression in BCa cells correlates with the capability to degrade ECM. We used a panel of characterized human BCa cell lines to determine if NEDD9 expression profiles identified in BCa patient samples correlate with invasion potential of cells,. We found that NEDD9 protein levels varied across the different cell lines in contrast to the p130Cas/BCAR1 protein (Fig.1D-E). Several BCa cell lines including MDA-MB-231, ZR-75-1, and BT-549 have 5 to 8 fold more NEDD9 when compared to the non-transformed MCF10A line. The high NEDD9 expression levels correlate with the invasive capabilities of these cells (31-32). We confirmed these findings using FITC-gelatin degradation assays. Representative images and quantification of degradation by MCF7, BT-549 and ZR-75-1 shown in Figure 1F-G and Suppl.Fig.1B. In contrast to the highly invasive MDA-MB-231 and BT-549, the AU-565 and MCF7 BCa cells were not proficient in gelatin degradation. We and others previously noticed that these cells expressed low levels of cortactin (Fig.1D), MMP14, and MMP2 (33) which are required for invasion (16). This indicates that NEDD9 is required to support ECM degradation, although other components of the invasion machinery are also required for these processes.

NEDD9 localizes to and promotes invadopodia maturation. To define the requirement of NEDD9 in formation and function of invadopodia, we infected MDA-MB-231, BT-549, and ZR75-1 cells with two different shRNAs targeting NEDD9, which did not effect the expression of p130Cas (Fig.2A). Cells were plated on FITC-gelatin coated coverslips and co-stained with 
invadopodia markers F-actin and cortactin to visualize the area of gelatin degradation and pseudopods (Fig.2B-C). Depletion of NEDD9 led to a two- threefold reduction in the number of cells degrading matrix, while viability of cells was not affected (Fig.2D). Interestingly, NEDD9 depleted cells had a similar number of F-actin/cortactin rich puncta (Fig.2E) which are found during the initial stages of invadopodia formation (16) but show an absence of degradation beneath the puncta (Fig.2C). p130Cas, which is highly expressed in BCa cells (Fig2A), was not able to compensate for the decrease in NEDD9 under current experimental settings suggesting a critical role of NEDD9 in invadopodia based invasion in BCa. The localization of other major components of invadopodia, such as: Arp3 and N-WASP, were not affected by depletion of NEDD9 (Suppl.Fig.1C). To determine if NEDD9 localized to invadopodia in BCa cells, we costained cells with anti- NEDD9 antibodies (29) and invadopodia markers (16). We found that NEDD9 was consistently co-localized with cortactin, actin, and phTyrosine (Fig.2F-G) in all cells with invadopodia.

NEDD9 depletion in BCa cells leads to decrease in 3D invasion. To assess the impact of NEDD9 depletion on ECM degradation under more physiological conditions we used matrigelDQcollagen I/IV invasion assay to trace invasion and pericellular ECM degradation in 3D matrix. BCa cells treated with shRNAs against NEDD9 were deficient in matrix degradation when embedded in 3D matrix (Fig.3A-C). The levels of FITC-fluorescence as a result of proteolytical cleavage of DQcollagen I/IV were calculated (Fig.3B) in shRNA expressing RFPpositive cells. The results were also evaluated in BT-549 and ZR-75-1 cell lines (Suppl.Fig.2AB). shNEDD9 cells demonstrated severe deficiency in collagen I/IV degradation indicated by a six fold decrease in fluorescence. We observed that the area of degradation in control cells had either a pericellular or dotted pattern (insert, Fig.3A).

\section{NEDD9 knockdown decreases MMPs activity, but does not affect MMPs expression or} secretion. MMPs are the key enzymes responsible for matrix degradation. To determine the potential impact of NEDD9 on the function of MMPs, we analyzed protein levels of MMPs in shNEDD9 and control cells using a comprehensive MMP antibody array. Serum-free medium (SFM) conditioned by control and shNEDD9 cells was collected to measure the levels of secreted MMPs. The whole cell lysates (WCL) were used to define the total protein level of various expressed MMPs. No significant differences in the total amount of produced or secreted MMP and tissue specific inhibitor of MMP- TIMPs, were detected (Fig.4A-B), except MMP13 which was slightly elevated in shNEDD9 cells. Nevertheless, analysis of SFM conditioned by 
shNEDD9, but not control, using EnzChek Gelatinase/Collagenase assay shows a severe deficiency in the activity of secreted collagenases (Fig.4C). Next, we tested the activity of secreted MMPs by gel zymography. The activity of MMPs, particularly MMP2 and MMP9, was reduced by $60-80 \%$ upon NEDD9 depletion (Fig.4D-E), indicated by the decrease in the amount of active $68 \mathrm{kDa}$ form of MMP2 (Fig.4D, quantification of 68kDa form Fig.4E) and 92-86kDa forms of MMP9 (Fig.4E). We then tested the activity of MMP14 using a MMP14-specific fluorogenic substrate and show that NEDD9 depletion results in a significant decrease in MMP14 activity (Fig.4F).

NEDD9 depletion leads to inactivation of MMP14 by TIMP2. Membrane bound MMP14 is one of the key molecules involved in activation of soluble MMPs and pericellular degradation (34). Total MMP14 protein level (Fig.5A) and localization patterns (Suppl.Fig.2C) were not impacted by NEDD9 depletion. Interestingly, cell surface levels of MMP14 and TIMP2 proteins assessed by FACS (Fig.5B-C) and IF (Fig.5D-E) had increased up to $40 \%$ upon NEDD9 depletion. Note, that according to the MMP array analysis performed earlier depletion of NEDD9 did not affect total expression or secretion of TIMP2 (Fig.4A-B). TIMP2 is a well-known regulator of MMP14 and MMP2 activity. An increased amount of TIMP2-bound MMP14 is indicative of its decreased activity (35-36). Depletion of MMP14 (Fig.6A) and TIMP2 in BCa cells (Fig.6B), using specific siRNAs, resulted in a similar to shNEDD9 invasion deficiency (Fig.6C) suggesting that MMP14 and TIMP2 are potentially the downstream targets affected by NEDD9. Moreover, addition of increasing amounts of recombinant TIMP2 to the medium of control cells leads to a similar inhibition of invasion at the higher concentrations of TIMP2 (Fig.6D). Importantly, reexpression of NEDD9 (Fig.6E) restored the amount of surface-bound MMP14 (Fig.6F) and invasion proficiency of shNEDD9 cells (Fig.6G).

NEDD9 depletion inhibits invasion and intravasation of tumor cells from primary site and growth of metastasis in xenograft models. To determine the requirements of NEDD9 for invasion and metastasis in vivo, we utilized human tumor xenografts combined with bioluminescence imaging (BLI) (27, 30). MDA-MB-231-shCon and shNEDD9 (N1, N4) cells were injected into the mammary fat pad and allowed to develop primary tumors without induction of shRNA. When tumor volume reached 150-200mm3 (Fig.7A-shRNA-Day0), animals were placed on doxycycline-containing food. shRNA expression was monitored by RFP fluorescence, along with BLI (Fig.7A-shRNA-Day14) and western blotting (Fig.7B). Animals were euthanized at shRNA Day0 or Day14 which corresponded to 3, and 5 weeks post injection 
of tumor cells to assess for the number of CTCs in the peripheral blood, number and size of metastases in the lungs and gross pathology of primary tumor. At the shRNA Day0, all animals had similar primary tumor size and volume based on caliper measurements and BLI

(Fig.7CshRNA-Day0). Analysis of peripheral blood samples for CTCs reveals similar number in all three cohorts of animals (shCon/shN1/shN4) (Fig.7D-shRNA-Day0), as well as similar number of metastases seeded in the lungs (Fig.7E-shRNA-Day0). NEDD9 depletion during the following 14 days in established primary tumors and metastases led to a drastic decrease in the number of CTCs (Fig.7D-shRNA-Day14) and number of metastases in lungs (Fig.7E-F-shRNADay14). The primary tumors had continued to grow during the shRNA induction based on BLI data (Fig.7C-shRNA-Day0/Day14); thus, the decrease in the number of CTCs could be either due to a decrease in the ability of tumor cells to invade the surrounding matrix and intravasate in blood stream or due to decrease in overall survival. We have established that NEDD9 depletion did not affect the survival of MDA-MB-231 cells (Fig.7G). Similar results were obtained with another metastatic BCa cell line BT-549 supporting the general phenomenon of NEDD9 function in invasion and metastasis (Suppl.Fig.3A-D). Collectively, our findings suggest that the application of shRNAs against NEDD9 to already established tumors could drastically reduce the number of tumor cells released into circulation due to an invasion deficiency, thereby, eliminating further seeding and decrease the total number of metastasis. 


\section{Discussion}

While recent studies have established NEDD9 as an essential factor for invasion (2-4) and metastasis $(1,37)$ the precise mechanism of how NEDD9 functions and its contribution to various stages of metastasis have been poorly defined. In our current study we show that NEDD9 is upregulated in breast tumors, and specifically increases in IDC, giving NEDD9 the potential to serve as a strong diagnostic marker for invasive tumors.

Interestingly, NEDD9 is decreased in metastatic samples suggesting that high level of NEDD9 is required during the invasion from the primary site, most probably to enable proteasedependent degradation of the basal membrane and EMT re-programming. While still increased compared to normal tissue, lesser amounts of NEDD9 are sufficient during growth at distant metastatic sites. Alternatively, upon arrival at distant sites, a decrease in NEDD9 expression might be beneficial in order to undergo mesenchymal to epithelial transition to establish the colony (8). These data is in accordance with the previously published studies showing that established metastasis in lungs had less NEDD9 when compared to primary tumor (6-7, 21-22). It is possible that decrease in NEDD9 might be beneficial for migration through filamentous collagen rich matrices; while in the basal membrane like matrix, the presence of NEDD9 was an absolute requirement for efficient invasion.

Decreases in NEDD9 expression, induced by constitutive or inducible shRNAs against NEDD9, result in a dramatic decrease in matrix degradation and invasion of $B C$ a cells in $2 D, 3 D$ environments, and limits number of CTCs and metastases, thus establishing NEDD9 as an important factor for invasion and metastasis in $\mathrm{BCa}$. In a search for a molecular mechanisms underlying NEDD9 dependent invasion, we found that depletion of NEDD9 does not affect formation of nascent invadopodia, characterized by aggregation of F-actin, cortactin, and MMP14 (17), but prevents further maturation of invadopodia into proteolytically active structures (16). These findings are in agreement with previous report in SCC-9 cells where NEDD9 expression was required for matrix degradation, but disagree with regard of NEDD9 requirement for invadopodia formation (23). The differences might be associated with artificial exogenous overexpression of NEDD9 in SCC9 cells. The source of differences could also reside in tissue specific behavior of breast versus head and neck cells. It is also needs to be noted that another NEDD9 family member protein p130Cas was previously identified in invadopodia (24). In the cell lines used in our study depletion of NEDD9 did not affect the formation of invadopodia, indicating the different roles of these adaptor proteins in invasion.

The maturation of invadopodia in BCa cells and the capacity of tumor cells to invade surrounding tissue are directly linked to accumulation and activation of MMP14 and soluble 
MMPs $(10,34)$. We found that depletion of NEDD9 leads to a $60-80 \%$ decrease in activity of secreted MMP2 and MMP9 and pericellular proteolysis. Activity of MMP14 is tightly regulated by TIMP2 which plays dual roles as both inhibitor and activator of MMP14, depending upon concentration (35-36). While there is no difference in produced or secreted protein levels of MMPs and TIMPs in shNEDD9 and shCon cells, we observed that the concentration of cell surface bound TIMP2 and MMP14 is up to 50\% higher in shNEDD9 cells, suggesting that the decrease in activity of MMP14 and other MMPs is potentially due to an increased amount of TIMP2 associated with MMP14 on the cell surface. Interestingly, depletion of MMP14 or TIMP2 results in a similar decrease in MMP2 activity and invasion deficiency recapitulating the shNEDD9 phenotype. TIMP2 is a potent inhibitor of most soluble MMPs (39), thus TIMP2 depletion could also relieve the inhibition of other soluble MMPs produced by cells.

Nevertheless, our findings indicate that depletion of TIMP2 alone is not sufficient to restore the activity of soluble MMPs most likely due to the presence of other TIMPs such as TIMP1, 3 and 4 (39-40). Re-expression of NEDD9 restores the MMPs activity and invasion proficiency of shNEDD9 cells.

Binding of TIMP2 results in increased endocytosis of MMP14 $(10,38)$ followed by dissociation of MMP14/TIMP2 complexes within the endocytic vesicles and regenerate an active protease (34). Accumulation of MMP14/TIMP2 complexes on the cell surface is indicative of endocytotic deficiency in NEDD9 depleted cells. Interestingly, MMP14 can be phosphorylated by Src kinase and this phosphorylation is required for invasion and endocytosis of MMP14 (19), thus directly linking the NEDD9 dependent decrease in Src and FAK activity $(4,21)$ with inactivation of MMP14. Further work is required to uncover the intricate link between NEDD9 and MMP14 trafficking.

The decrease in number of metastases and CTCs upon NEDD9 depletion in cancer cells could advocate for development of shRNA based NEDD9-targeted therapy against BCa invasion. Importantly, even established, late stage, cancers with seeded metastases based on our in vivo inducible xenograft studies might still benefit from shNEDD9 treatment and prevent further spreading of disease. Collectively, our findings establish a critical role for NEDD9 as a regulator of invasion in $\mathrm{BCa}$ through modulation of MMP14 activity, thus delineating a new signaling pathway for targeting NEDD9 dependent invasion. 


\section{References}

1. Kim M, Gans JD, Nogueira C, Wang A, Paik JH, Feng B et al. Comparative oncogenomics identifies NEDD9 as a melanoma metastasis gene. Cell 2006; 125:1269-1281.

2. Kondo S, Iwata S, Yamada T, Inoue Y, Ichihara H, Kichikawa Y et al. Impact of the integrin signaling adaptor protein NEDD9 on prognosis and metastatic behavior of human lung cancer. Clin Cancer Res. 2012; 18:6326-6338.

3. Natarajan M, Stewart JE, Golemis EA, Pugacheva EN, Alexandropoulos K, Cox BD et al. HEF1 is a necessary and specific downstream effector of FAK that promotes the ofglioblastoma cells. Oncogene 2006; 25:1721-1732.

4. Ahn J, Sanz-Moreno V, Marshall CJ. The metastasis gene NEDD9 product acts through integrin beta3 and Src to promote mesenchymal motility and inhibit amoeboid motility. J Cell Sci. 2012; 125:1814-1826.

5. Fashena SJ, Einarson MB, O'Neill GM, Patriotis C, Golemis EA. Dissection of HEF1dependent functions in motility and transcriptional regulation. J Cell Sci. 2002;115: 99111.

6. Guerrero MS, Parsons JT, Bouton AH. Cas and NEDD9 Contribute to Tumor Progression through Dynamic Regulation of the Cytoskeleton. Genes Cancer. 2012; 3:371-381.

7. Sanz-Moreno V, Gadea G, Ahn J, Paterson H, Marra P, Pinner S et al. Rac activation and inactivation control plasticity of tumor cell movement. Cell. 2008; 135:510-523.

8. Kong C, Wang C, Wang L, Ma M, Niu C, Sun X et al. NEDD9 is a positive regulator of epithelial-mesenchymal transition and promotes invasion in aggressive breast cancer. PLoS One 2011; 6(7):e22666. doi: 10.1371.

9. Egeblad M, Werb Z. New functions for the matrix metalloproteinases in cancer progression. Nat Rev Cancer 2002; 2:161-174.

10. Poincloux R, Lizarraga F, Chavrier P. Matrix invasion by tumour cells: a focus on MMP14 trafficking to invadopodia. J Cell Sci. 2009; 122:3015-3024.

11. Osenkowski $P$, Toth M, Fridman R. Processing, shedding, and endocytosis of membrane type1-matrix metalloproteinase (MMP14). J Cell Physiol. 2004; 200:2-10.

12. Yana I and Weiss SJ. Regulation of membrane type-1 matrix metalloproteinase activation by proprotein convertases. Mol Biol Cell. 2000; 11(7):2387-401.

13. Sato H, Kinoshita T, Takino T, Nakayama K, Seiki M. Activation of a recombinant membrane type 1-matrix metalloproteinase (MT1-MMP) by furin and its interaction with tissue inhibitor of metalloproteinases (TIMP)-2.FEBS Lett. 1996; 393(1):101-4.

14. Shofuda K, Moriyama K, Nishihashi A, Higashi S, Mizushima H, Yasumitsu $H$ et al. Role of tissue inhibitor of metalloproteinases-2 (TIMP-2) in regulation of pro-gelatinase A activation catalyzed by membrane-type matrix metalloproteinase-1 (MT1-MMP) in human cancer cells. J Biochem. 1998; 124(2):462-70.

15. Hernandez-Barrantes S, Toth M, Bernardo MM, Yurkova M, Gervasi DC, Raz Y et al. Binding of active ( $57 \mathrm{kDa}$ ) membrane type 1-matrix metalloproteinase (MMP14) to tissue inhibitor of metalloproteinase (TIMP)-2 regulates MMP14 processing and pro-MMP-2 activation. J Biol Chem. 2000; 275:12080-12089.

16. Artym VV, Zhang Y, Seillier-Moiseiwitsch F, Yamada KM, Mueller SC. Dynamic interactions of cortactin and membrane type 1 matrix metalloproteinase at invadopodia: defining the stages of invadopodia formation and function. Cancer Res. 2006; 66:30343043.

17. Ayala I, Baldassarre M, Giacchetti G, Caldieri G, Tete S, Luini A et al. Multiple regulatory inputs converge on cortactin to control invadopodia biogenesis and extracellular matrix degradation. J Cell Sci. 2008; 121:369 378.

18. Bowden ET, Onikoyi E, Slack R, Myoui A, Yoneda T, Yamada KM et al. Co-localization of cortactin and phosphotyrosine identifies active invadopodia in human breast cancer cells. Exp Cell Res. 2006; 312:1240-1253. 
19. Nyalendo C, Beaulieu E, Sartelet H, Michaud M, Fontaine N, Gingras D et al. Impaired tyrosine phosphorylation of membrane type 1-matrix metalloproteinase reduces tumor cell proliferation in three-dimensional matrices and abrogates tumor growth in mice. Carcinogenesis 2008; 29:1655-1664.

20. Wu X, Gan B, Yoo Y, Guan JL. FAK-mediated src phosphorylation of endophilin A2 inhibits endocytosis of MMP14 and promotes ECM degradation. Dev Cell. 2005; 9:185196.

21. Izumchenko E, Singh MK, Plotnikova OV, Tikhmyanova N, Little JL, Serebriiskii IG et al. NEDD9 promotes oncogenic signaling in mammary tumor development. Cancer Res. 2009; 69:7198-7206.

22. Little JL, Serzhanova V, Izumchenko E, Egleston BL, Parise E, Klein-Szanto AJ et al. A requirement for Nedd9 in luminal progenitor cells prior to mammary tumorigenesis in MMTVHER2/ErbB2 mice. Oncogene 2013; doi: 10.1038/onc.2012.607.

23. Lucas JT, Jr., Salimath BP, Slomiany MG, Rosenzweig SA Regulation of invasive behavior by vascular endothelial growth factor is HEF1-dependent. Oncogene 2010; 29: 4494459.

24. Brabek J, Constancio SS, Siesser PF, Shin NY, Pozzi A, Hanks SK. Crk-associated substratetyrosine phosphorylation sites are critical for invasion and metastasis of SRCtransformed cells. Mol Cancer Res. 2005; 3:307-315.

25. Wang $Y$ and McNiven MA. Invasive matrix degradation at focal adhesions occurs via protease recruitment by a FAK-p130Cas complex. J Cell Biol. 2012; 196(3):375-85.

26. Pugacheva EN, Jablonski SA, Hartman TR, Henske EP, Golemis EA. HEF1-dependent Aurora A activation induces disassembly of the primary cilium. Cell 2007; 129:13511363.

27. Zinn KR, Chaudhuri TR, Szafran AA, O'Quinn D, Weaver C, Dugger K, et al. Noninvasive bioluminescence imaging in small animals. ILAR J. 2008; 49:103-15.

28. Jedeszko C, Sameni M, Olive MB, Moin K, Sloane BF. Visualizing protease activity in living cells: from two dimensions to four dimensions. Curr Protoc Cell Biol. 2008; Chapter 4: Unit 20.

29. Pugacheva EN, Golemis EA. The focal adhesion scaffolding protein HEF1 regulates activation of the Aurora-A and Nek2 kinases at the centrosome. Nat Cell Biol. 2005; 7:937-946.

30. Ice RJ, McLaughlin SM, Culp MV, Eddy ER, Livengood RH, Ivanov AV, et al. NEDD9 depletion destabilizes Aurora A kinase and heightens the efficacy of Aurora A inhibitors: implications for treatment of metastatic solid tumors. Cancer Res. 2013;73(10):3168-80.

31. Madsen MW, Briand P. Relationship between tumorigenicity, in vitro invasiveness, and plasminogen activator production of human breast cell lines. Eur J Cancer. 1990; 26:793-797.

32. Ratnikov BI, Deryugina EI, Strongin AY. Gelatin zymography and substrate cleavage assays of matrix metalloproteinase-2 in breast carcinoma cells overexpressing membrane type1 matrix metalloproteinase. Lab Invest. 2002; 82:1583-1590.

33. Sounni NE, Devy L, Hajitou A, Frankenne F, Munaut C, Gilles C et al. MMP14 expression promotes tumor growth and angiogenesis through an up-regulation of vascular endothelial growth factor expression. FASEB J. 2002; 16:555-564.

34. Li XY, Ota I, Yana I, Sabeh F, Weiss SJ. Molecular dissection of the structuralmachinery underlying the tissue-invasive activity of membrane type-1 matrix metalloproteinase. Mol Biol Cell 2008; 19:3221-3233.

35. Strongin AY, Collier I, Bannikov G, Marmer BL, Grant GA, Goldberg GI. Mechanism of cell surface activation of 72-kDa type IV collagenase. Isolation of the activated form of the membrane metalloprotease. J Biol Chem. 1995; 270:5331-5338.

36. Fernandez-Catalan C, Bode W, Huber R, Turk D, Calvete JJ, Lichte A et al. Crystal 
structure of the complex formed by the membrane type 1-matrix metalloproteinase with the tissue inhibitor of metalloproteinases-2, the soluble progelatinase A receptor. EMBO J. 1998; 17:5238-5248.

37. O'Neill GM, Seo S, Serebriiskii IG, Lessin SR, Golemis EA. A new central scaffold for metastasis: parsing HEF1/Cas-L/NEDD9. Cancer Res. 2007; 67:8975-8979.

38. Remacle A, Murphy G, Roghi C. Membrane type I-matrix metalloproteinase (MMP14) is internalised by two different pathways and is recycled to the cell surface. J Cell Sci. 2003; 116:3905-3916.

39. Brew K, Nagase H. The tissue inhibitors of metalloproteinases (TIMPs): an ancient family with structural and functional diversity. Biochimica Biophysica Acta 2010; 1803: 55-71.

40. Zhang M, Liu Y, Feng H, Bian X, Zhao W, Yang Z, et al. CD133 affects the invasive ability of HCT116 cells by regulating TIMP-2. Am.J.Pathol. 2013; 182(2):565-76. 


\section{Figure legends}

Figure 1. NEDD9 is overexpressed in invasive breast ductal carcinomas and BCa cell lines. (A). Representative images of IHC staining with anti-NEDD9 antibodies in normal breast, DCIS, IDC MIDC-LN. (B). Statistical analysis of NEDD9 expression based on intensity of staining in (A), mean values +/-S.E.M, one-way ANOVA*, p<0.001 (Normal/IDC, Normal/MIDC LN, Normal/DCIS), p<0.05 (DCIS/IDC, IDC/MIDC-LN), ns-non significant (DCIS/MIDC-LN) (C). The data $(B)$ were analyzed by Random forest statistical software to assess the predictive value of NEDD9 expression. (D). Western Blot analysis (WB) of NEDD9, p130Cas, cortactin and tubulin in the indicated cell lines. (E). Quantification of NEDD9 and p130Cas as in (D), normalized to MCF10A, * $p<0.001$. (F). Immunofluorescent (IF) staining of MCF7 and BT-549 cells with Rhodamine-Phalloidin (red) and cortactin (green), FITC-gelatin (white). (G). Quantification of matrix degradation, 100 cells/cell line $n=3, \%$ of cells degrading matrix, $+/-$ S.E.M, * $p<0.0001$ one-way ANOVA.

Figure 2. NEDD9 localizes to and promotes invadopodia maturation. (A). Top: WB of NEDD9, p130Cas, actin in MDA-MB-231-shNEDD9 or -shCon cells, Bottom: quantification of NEDD9 and $p 130$ Cas expression normalized to shCon; one-way ANOVA, $p<0.001$. (B). IF staining of MDA-MB-231-shCon and -shNEDD9 cells with anti-cortactin (green), -actin (red) antibodies and FITC-gelatin (blue), scale bar 10 main panel. (C). Enlarged XYZ projections of IF as in (B). (D). Quantification of number of cells degrading matrix and F-actin/cortactin positive foci (E) in MDA-MB-231-shCon, - shNEDD9 (N1, N4) cells. Student's t-test for (D): *p<0.001(shCon/shN1, shCon/shN4), ns - shN1/N4; and ns (E) +/-S.E.M. (F). IF of MDA-MB-231 cells with cortactin (blue), actin (red) and NEDD9 (green), and FITC-gelatin (white). (G). IF staining with anti-phTyr (blue), actin (red) and NEDD9 (green) antibodies, and FITC-gelatin (white). Scale bar $-\square \square \square \mathrm{m}$. Ins erts arenlarged areas indicated in the main panel.

Figure 3. NEDD9 depletion ablates matrix degradation and invasion in 3D matrices. (A). Representative images of 3D projections live cell imaging of MDA-MB-231-shCon, -shNEDD9 cells embedded in the DQ-CollagenI/IV/matrigel matrix, shRNA (red), DNA (blue) at indicated time points; xyz projections (z), scale bar -50 rectangle. (B). Quantification of FITC-fluorescence intensity as in (A), \% of area degraded per cell, +/-S.E.M, ${ }^{*} p<0.0001$ one-way ANOVA. (C). WB of cells in (A-B) with anti-NEDD9 and actin antibodies. +Dox indicates the lines used in A-B. 
Figure 4. NEDD9 depletion decreases MMPs activity, but does not affect MMPs expression or secretion. (A). WB of total MMPs expressed (WCL) and secreted (SFM) by indicated cells, 48h post shRNA expression. Schematic outline the MMP microarray. (B). Quantification of MMPs and TIMPs expression, $n=3$, one-way ANOVA for all pairs $p$ is non significant; $\mathrm{p}<0.05$ for MMP13 (shCon/shN4). (C). Quantification of gellatinase/collagenese activity in SFM conditioned by cells using EnzChek Gellatinase/Collagenese assay. Clostridium Collagenase, $0.1 \mathrm{U} / \mathrm{ml}$, is positive control, $\mathrm{p}<0.05$ (shCon/shN1 or $/ \mathrm{N} 4$ ), linear regression analysis. (D). Representative image of -gel zymography, SFM conditioned by MDA-MB-231 shCon, - shNEDD9 cells, WB of MMP2 and MMP9. (E). Quantification MMP2 and MMP9 activity as in (D) normalized to total level of MMP2 and MMP9, +/-S.E.M; MMP9: $p=0.007\left(^{*}\right)$ shCon/N1, or /N4 and ns - shN1/N4, one-way ANOVA. MMP2: $p=0.00031$, shCon/N1, /N4, ns -shN1/N4, one-way ANOVA. (F). MMP14-specific fluorogenic substrate assay, at 328/400mn. Data plotted as curves described by equation for one site binding kinetics, fitted to each data set with the R2 of 0.8 , Extra sum-of-squares $F$ test, $p<0.0001$.

Figure 5. NEDD9 depletion leads to increase in amount of surface-bound TIMP2 and MMP14: (A). WB of WCL with anti-NEDD9, -MMP14, -GAPDH antibodies. (B). FACS of MMP14: surface (non-permeabilized) and total (permeabilized) normalized to shCon; one-way ANOVA * $p<0.005$ (shCon/N1 or /N4) -surface cells, ns, -total. (C). FACS of TIMP2-surface, normalized to shCon; t-test ${ }^{*} p=0.039$ (shCon/N1), * $p=0.0029$ (shCon/N4). (D). Representative images of IF, MDA-MB-231-shCon, -shNEDD9 cells with anti-TIMP2 antibody (red), DNA (blue). Scale bar, 20

(E)) Qubatification100 magnified are of TIMP2 fluorescence intensity at the membrane as in (D) normalized to shCon; t-test ${ }^{*} p<0.001$ (shCon/shN1 or /N4), ns - shN1/shN4.

Figure 6. Inhibition or depletion of MMP14 or TIMP2 recapitulates invasion deficiency of shNEDD9 cells. (A-B). WB of WCL of MDA-MB-231-shCon, -shNEDD9 cells treated with siCon, siMMP14 or siTIMP2, using anti- NEDD9, -TIMP2 and -a-tubulin antibodies. (C). Boyden Chamber invasion assay, cells as in (AB); plotted as relative fluorescence units (RFU), one-way ANOVA * $\mathrm{p}<0.0001$ (shCon/siCon, shN1/siCon, or shN4/siCon), ns -shN1/N4-siCon and shCon/siMMP14 or shCon/siTIMP2; p<0.005 shCon/siCon, shCon/siMMP14 or shCon/siTIMP2; (D). Boyden Chamber invasion assay of MDA-MB-231-shCon cells with increased amount of TIMP2. MDA-MB-231-shN4 cells used as a reference control. Results plotted as RFU, n=3. 
One-way ANOVA * $\mathrm{p}<0.0001$ shCon-0nM/shCon-10nM or more, ns: shN4/shCon-10nM. (E). WB of NEDD9 rescue in MDAMB- 231-shCon, -shNEDD9 cells with empty vector or CDNA-NEDD9 construct, using anti- NEDD9, GAPDH antibodies. (F). FACS of MMP14 surface staining as in (E); one-way ANOVA * $p<0.0001$ empty vector-shCon/shN1 or /N4; ns CDNANEDD9 shCon/shN1 or /N4). (G). Boyden-Chamber assay, cells as in (E-F). Data plotted as RFU, one way ANOVA * $p<0.0001$ (shCon-empty/shCon-NEDD9, shN1-empty/shN1-NEDD9, shN4 empty/shN4- NEDD9); $p<0.005$ (shCon-empty/shN1-empty or -shN4-empty); ns (shCon NEDD9/shN1- NEDD9 or /shN4-NEDD9).

Figure 7. NEDD9 depletion inhibits metastasis in xenograft models. (A). Representative images of bioluminescence and RFP fluorescence of mice orthotopically injected with MDAMB231LN-shCon or -shNEDD9 cells. shRNA-Day0-3 weeks post injection, regular chow; shRNA Day14-2 weeks doxycycline chow; 6 mice/shRNA/3 independent experiments. (B). WB of primary tumor tissue at (shRNA-Day0, -Day14) with anti-NEDD9, -RFP and -actin antibodies. (C). Quantification of tumor growth based on bioluminescence in 3 independent experiments, $n=6 / g r o u p$, mean photon flux, +/-S.E.M, one-way ANOVA, $\mathrm{p}$ is ns for shRNADay0 (shCon/shN1 or /N4), shRNA-Day14 (shCon/shN1, shN1/shN4); ${ }^{*} \mathrm{p}=0.032$ for shRNADay14- shCon/shN4. (D). Quantification of RFP/luc2/DNA positive tumor cells in peripheral blood of shCon and shNEDD9 mice at indicated times, $n=6 /$ group/3 independent experiments; plotted as mean number of cells per $200 \quad$ SIE.:N6,1Wwo;-way ANOVA, ns (shRNADay0: shCon/shN1 or /N4); $p<0.001$ (shRNA-Day14: shCon/shN1 or /N4). (E). Quantification of metastases in lungs of orthotopically injected animals at indicated times by pathologist using serial sections of lungs and H\&E staining, plotted as mean number of metastases per mm2 +/- S.E.M, $n=6 /$ group/3 independent experiments; two-way ANOVA $p$-ns (shRNA-Day0: shCon/shN1 or /N4); $p<0.001$ (shRNA-Day14: shCon/shN1 or /N4). (F). Representative images of H\&E staining as in (E). Scale bar-500 Einsenta (G) pQuantifocation of number of trypan blue negative vs. positive shCon or shNEDD9 cells, 72 in suspension. One- way ANOVA, $p=0.0946$.

Supplementary Figure 1. (A). Statistical analysis of NEDD9 expression based on intensity and number of positively stained cells in Fig.1(A) visually determined by two pathologists according to the scoring procedure described in Material and Methods section. (B). Immunofluorescent (IF) staining of ZR-75-1 cells with Rhodamine-Phalloidin (red) and cortactin (green), FITCgelatin (pseudo colored white). (C). Immuofluorescent analysis of structural components of 
invadopodia: Arp3 (green), N-WASP (green), cortactin (red), DNA (blue), FITC-gelatin (white) in MDA231- MB-231-shCon, -shNEDD9 (N4) cells.

Supplementary Figure 2. (A). Representative images of live microscopy of BT549-shCon, shNEDD9 (N4) cells embedded in DQ-Collagen IV 48h post plating. DNA (blue), shRNA expressing cells (RFP, red) and degradation of DQ-Collagen (green). (B). Quantification of DQCollagen IV degradation as shown in (A). Statistical analysis by one-way ANOVA based on 3 independent experiments in 100-200 cells in each group. ${ }^{*} p<0.005$ (shCon/shN1 or N4), ns (shN1/N4). (C). IF analysis of MDA-MB-231-shCon, -shNEDD9 cells with anti-cortactin (blue),

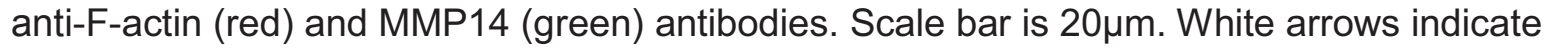
membrane area with increase in MMP14.

Supplementary Figure 3. (A). Representative images of bioluminescence (BLI) of mice orthotopically injected with BT-549 cells expressing doxycycline inducible shCon or shNEDD9 (shN1) RNAs. shRNA-Day0 represents 2 weeks post injection, before addition of doxycycline chow; shRNA-Day14 is 2 weeks post addition of doxycycline-containing diet. 6 mice/each shRNA (12 mice total). (B). WB (right panel) analysis of primary tumor tissue dissected at shRNA-Day14 with anti-NEDD9, and -GAPDH antibodies. Quantification of sRNA depletion 2 (left panel) from tumors from 3 mice/per shRNA, t-test ${ }^{*} p=0.0019$. (C). Quantification of tumor growth based on BLI (shRNA-Day0, -Day14) in 3 independent experiments, $n=6 /$ group, mean photon flux, +/-S.E.M, two-way ANOVA, p values are non-significant (ns) for shRNA-Day0 (shCon/shN1); ${ }^{*}=0.0007$ for shRNA-Day14 (shCon/shN1). (D). Quantification of RFP/luc2/DNA positive tumor cells in peripheral blood of shCon and shNEDD9 (N1) mice at indicated time

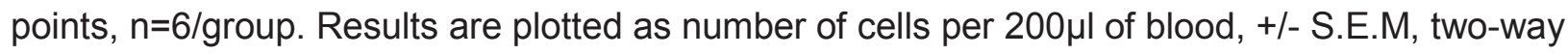
ANOVA, ns (shRNA-Day0: shCon/shN1); $p<0.001$ (shRNA-Day14: shCon/shN1). 


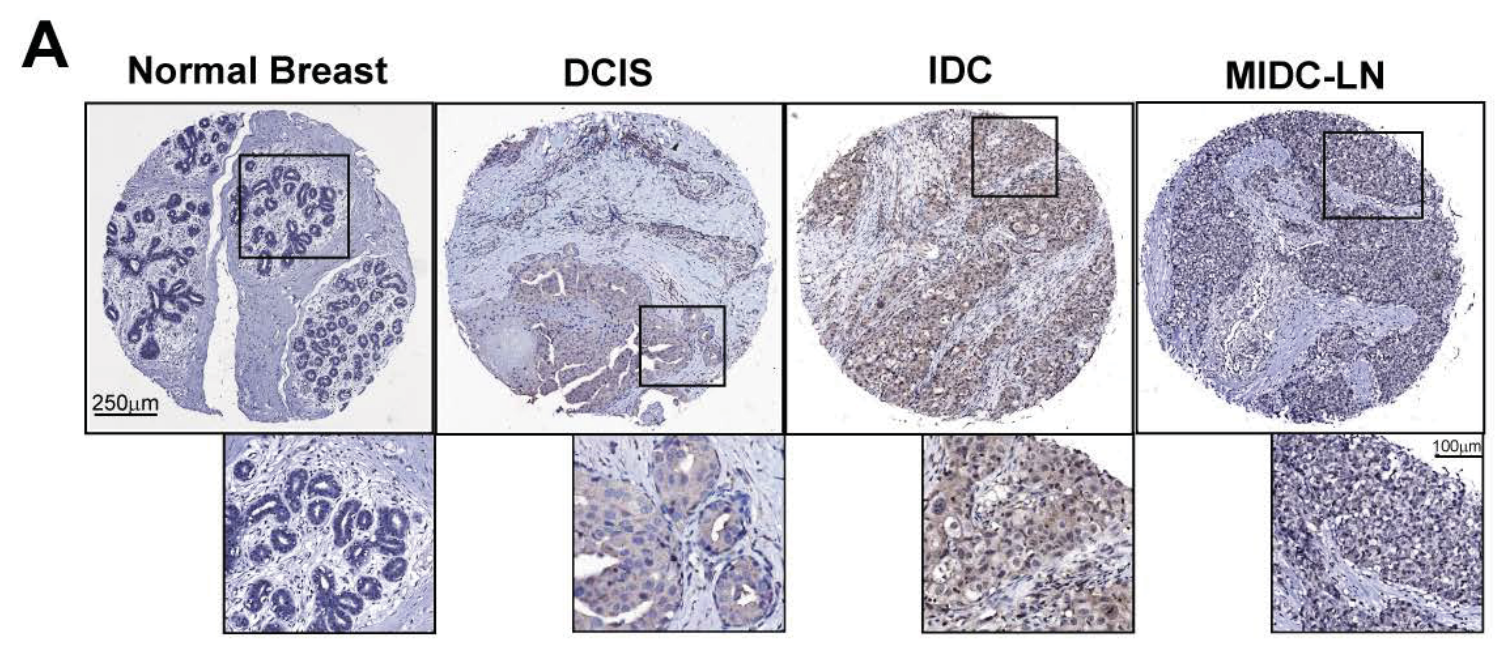

B
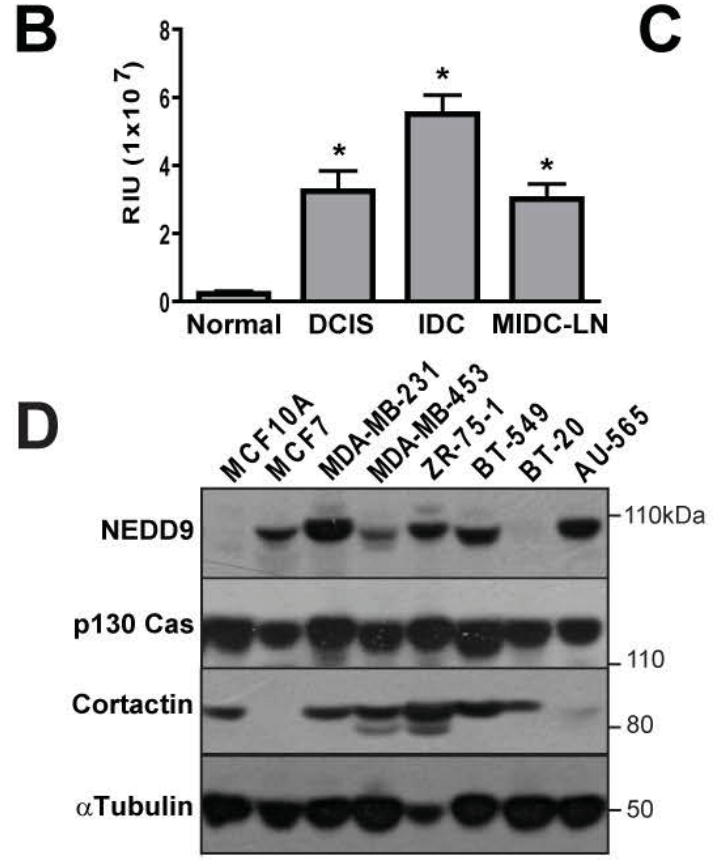

C

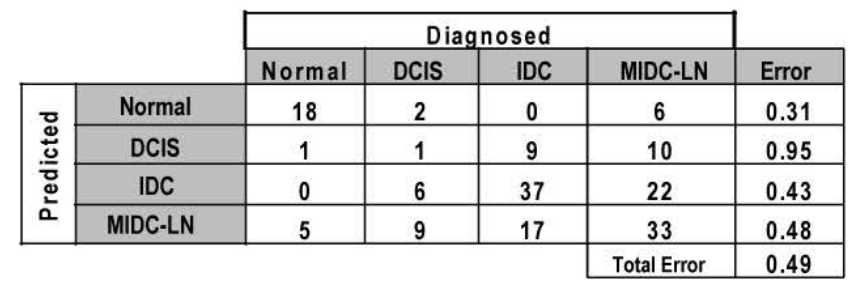

E

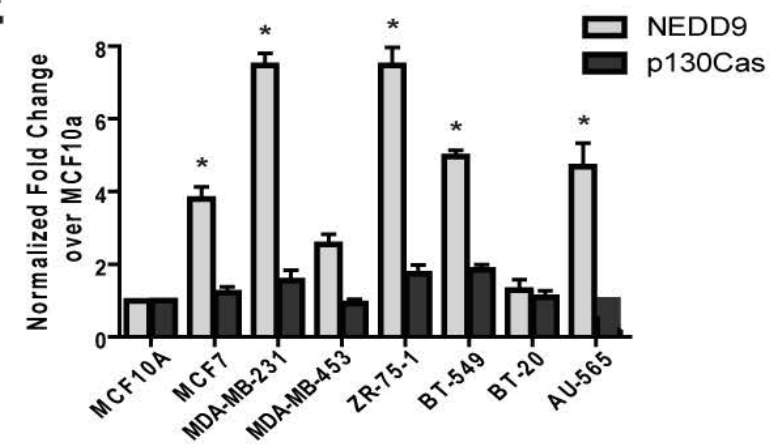

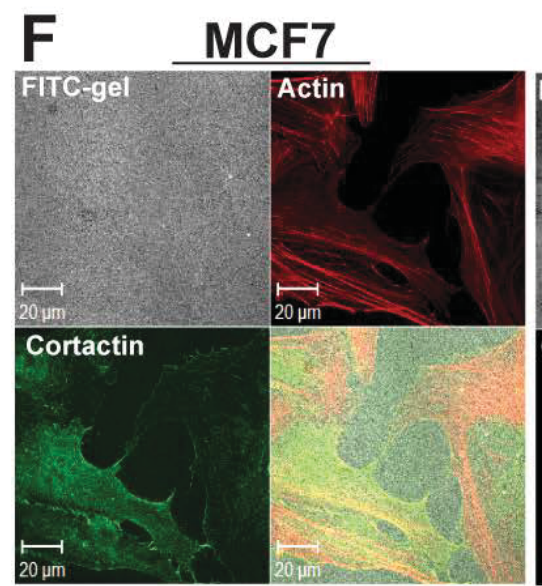

BT-549

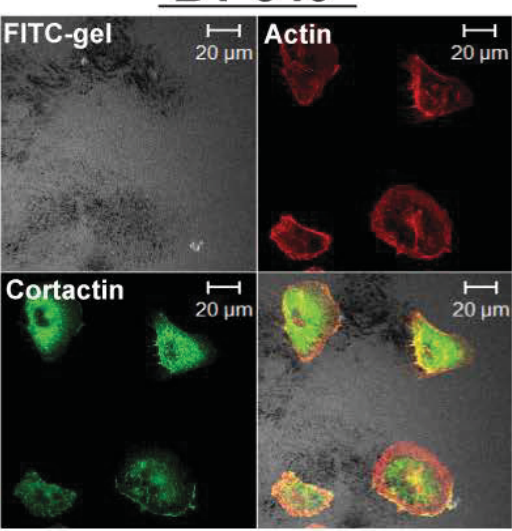

$\mathbf{G}$

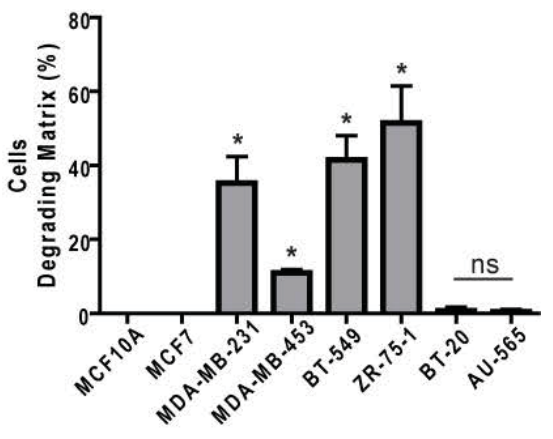

Figure 1, McLaughlin 

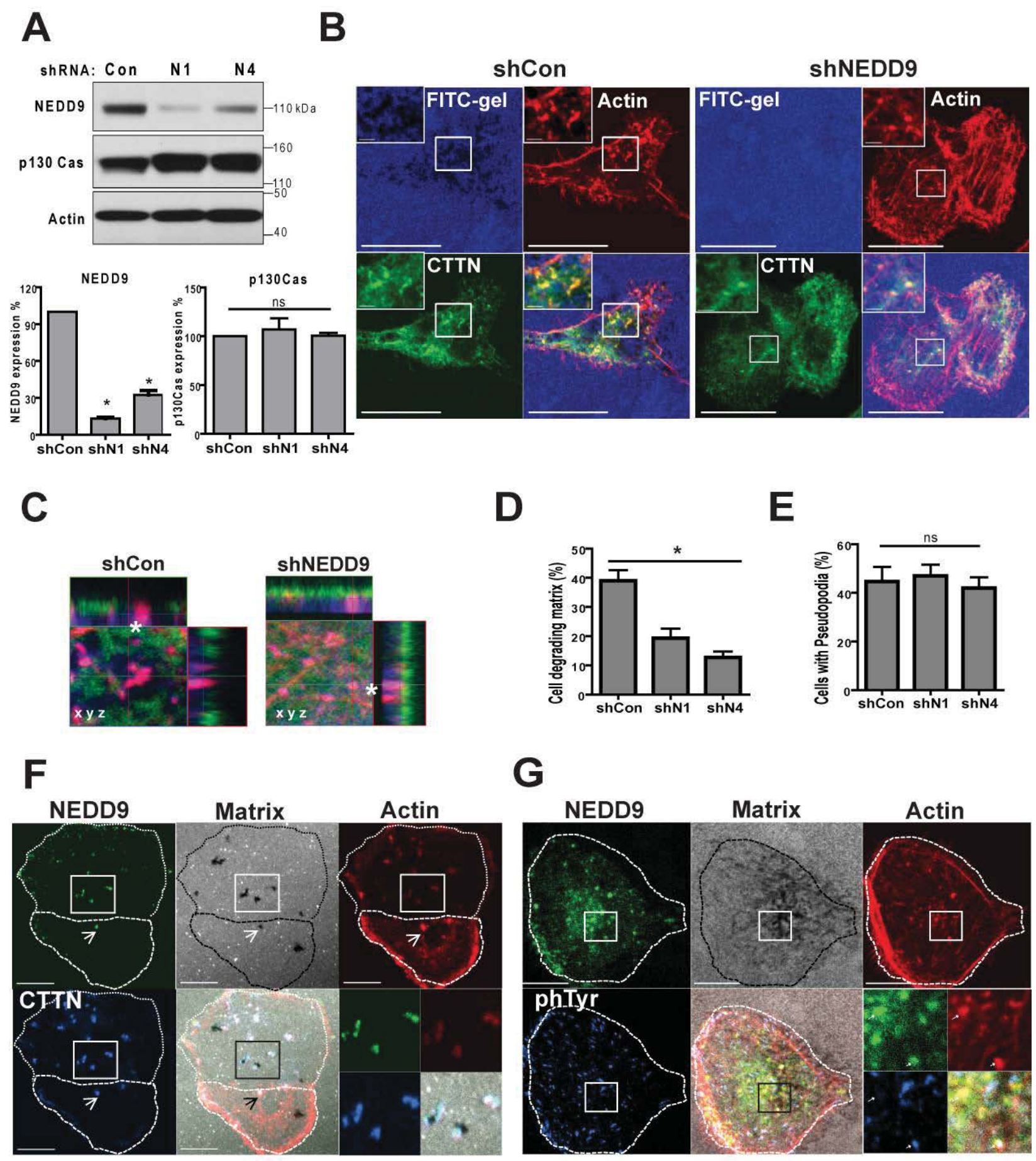

G

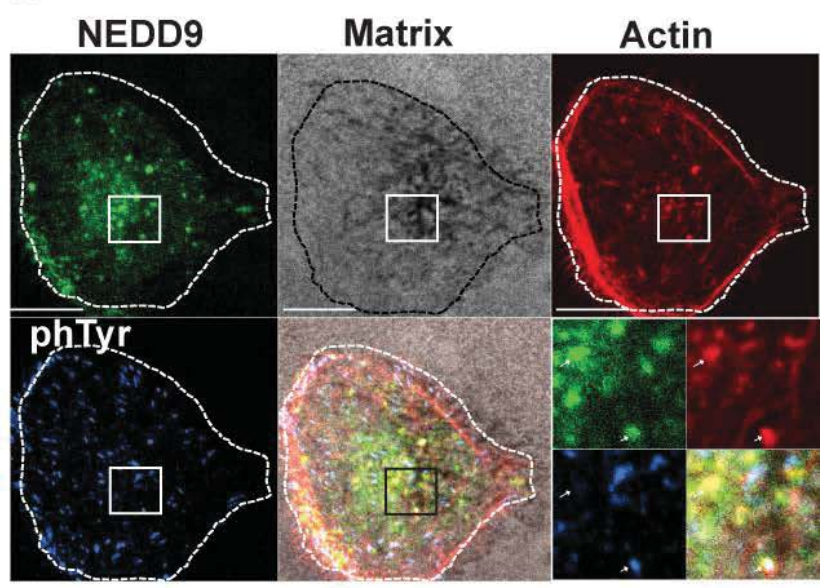

Figure 2, McLaughlin 
A

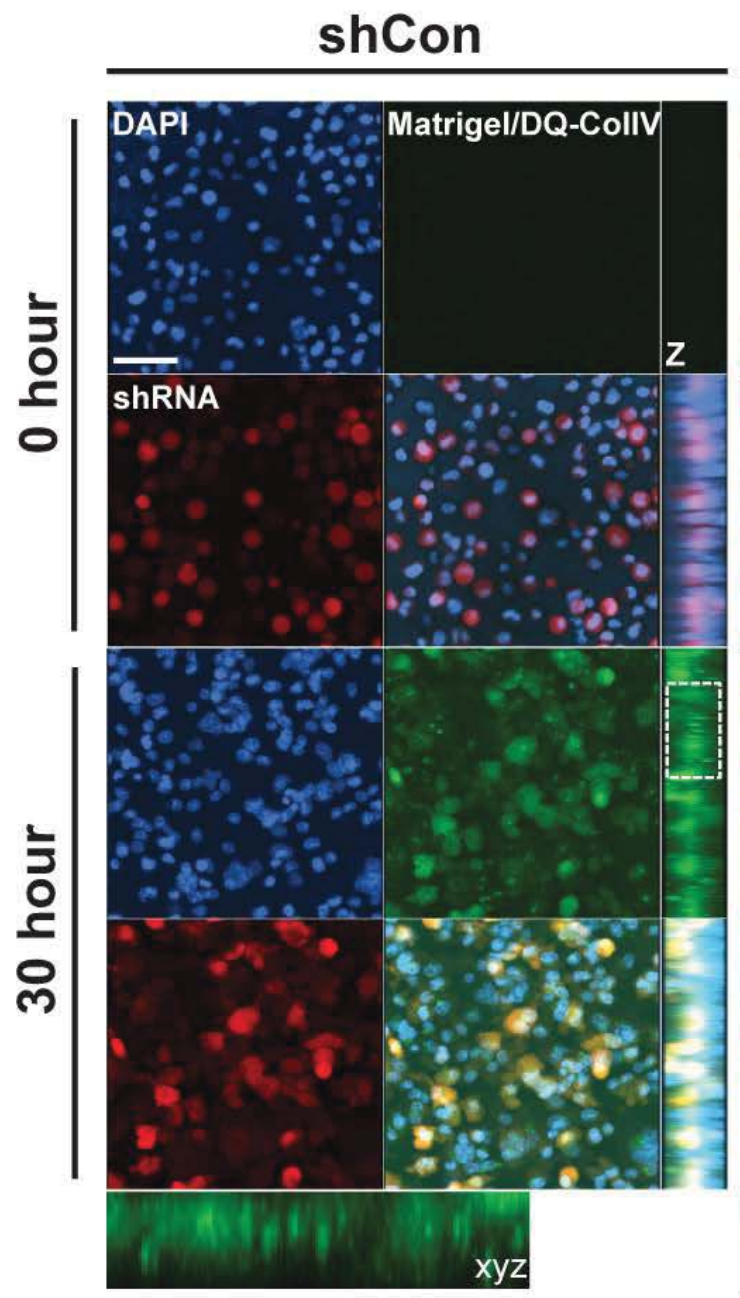

B

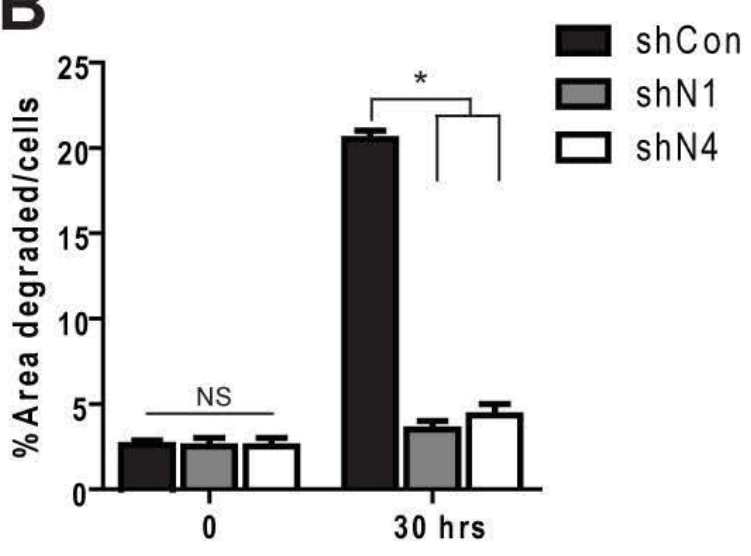

shNEDD9
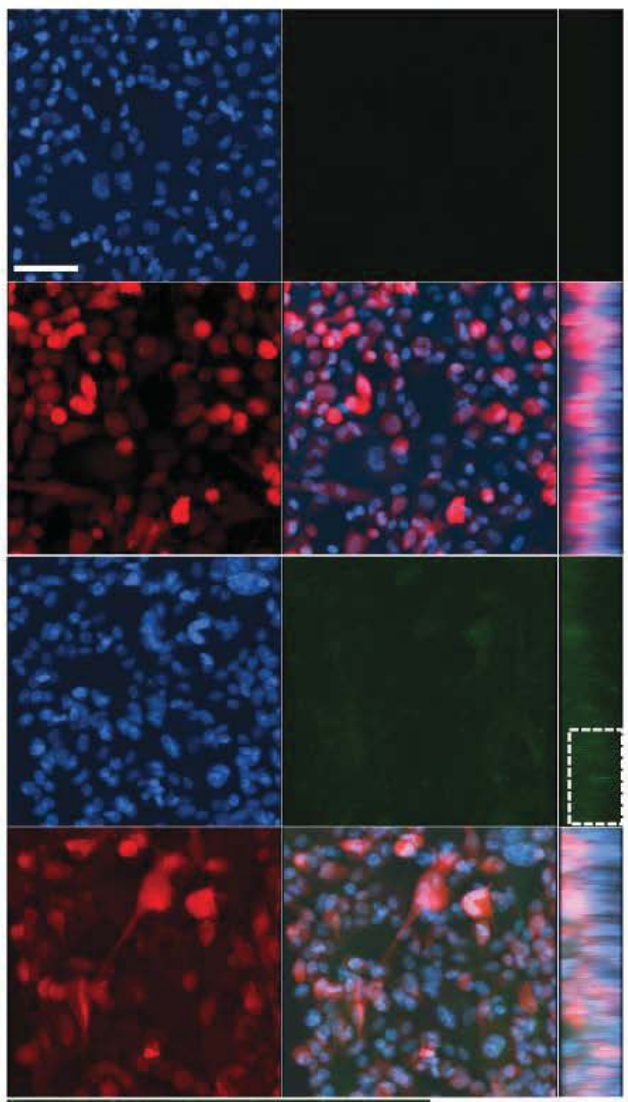

xyz

C

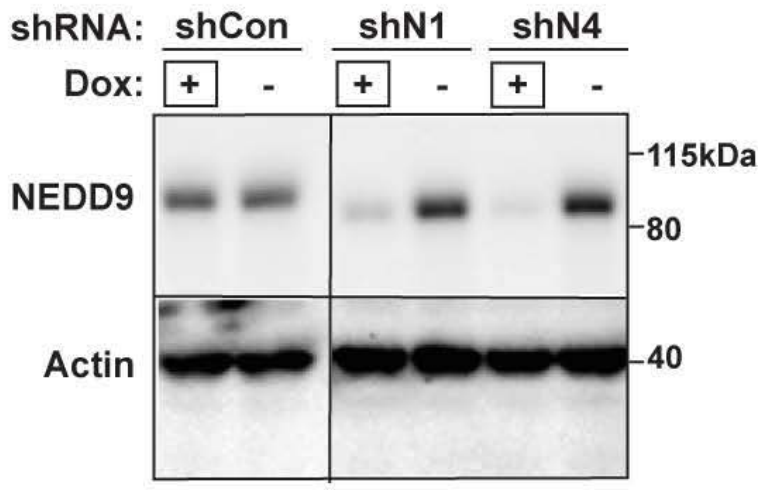

Figure 3, McLaughlin 


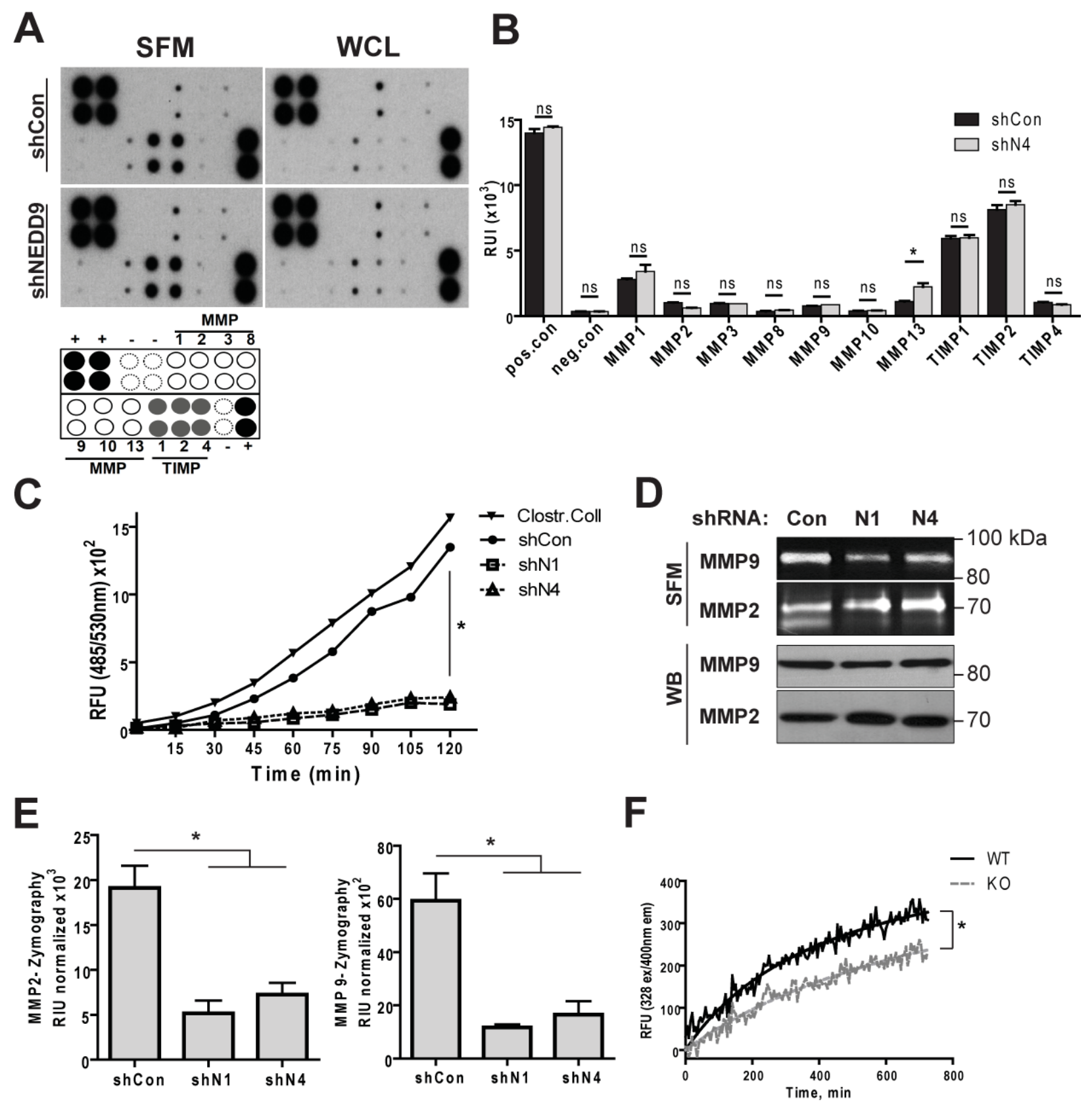

Figure 4, McLaughlin 


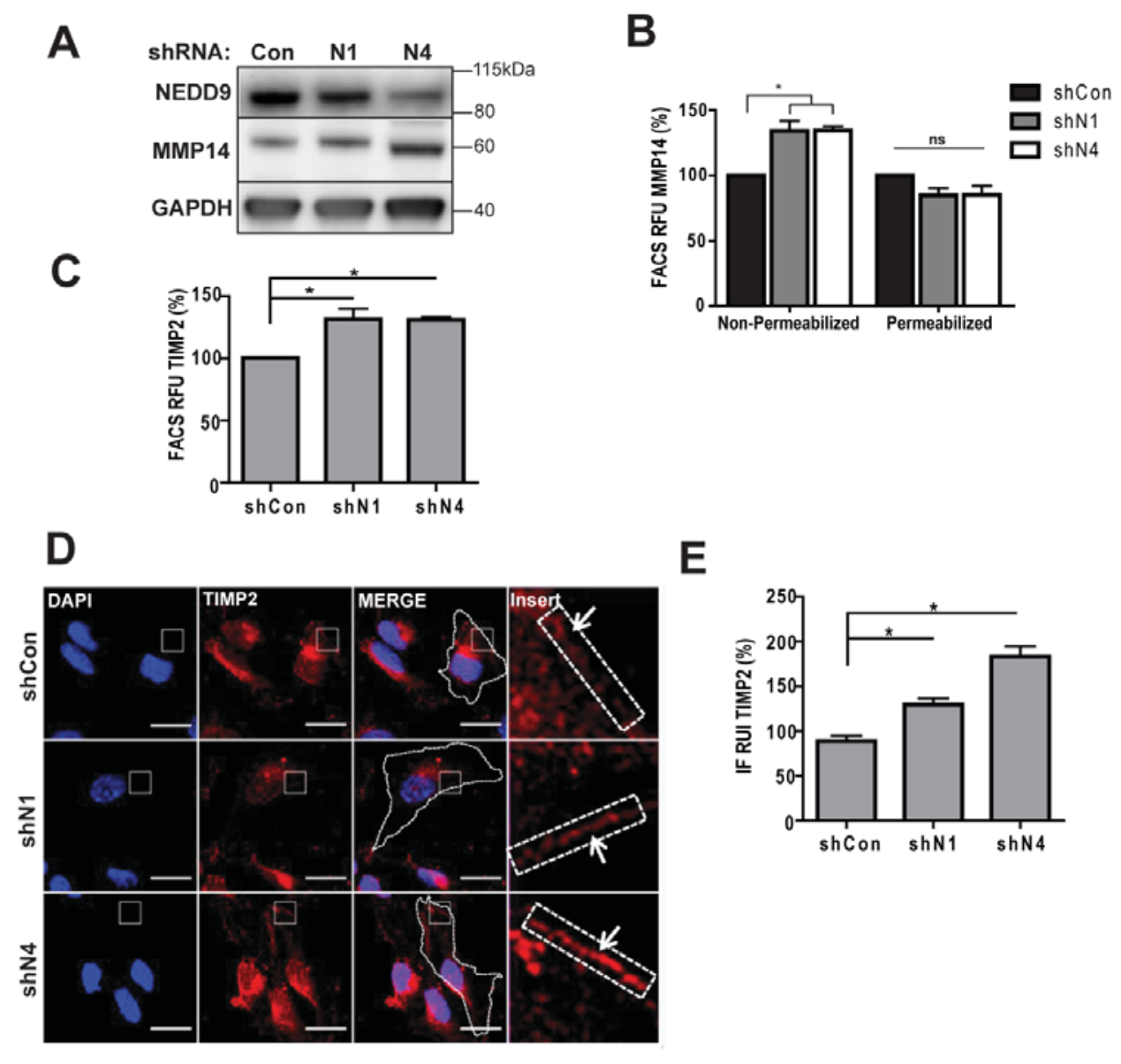

Figure 5, McLaughlin 


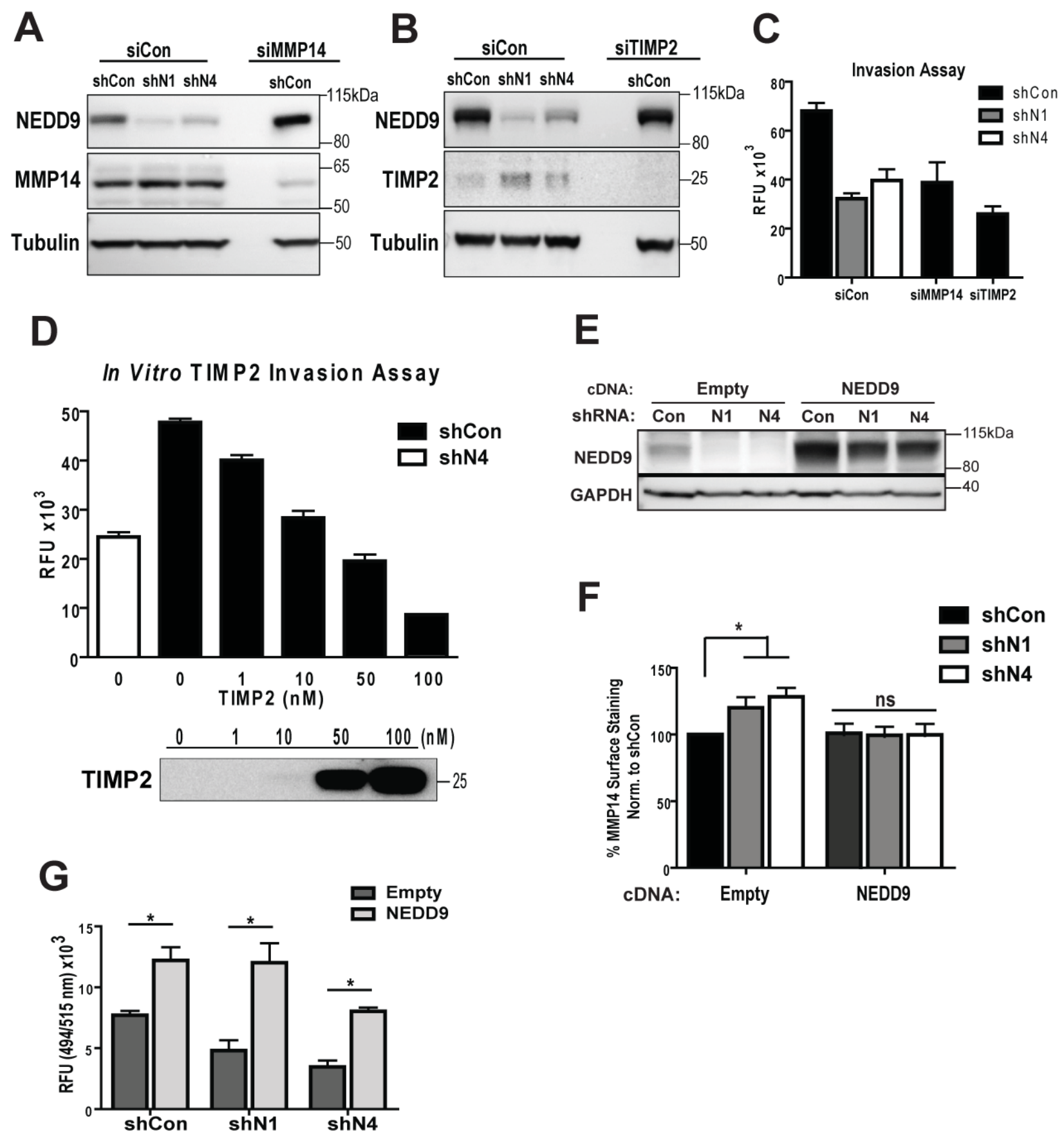

Figure 6, McLaughlin 
A
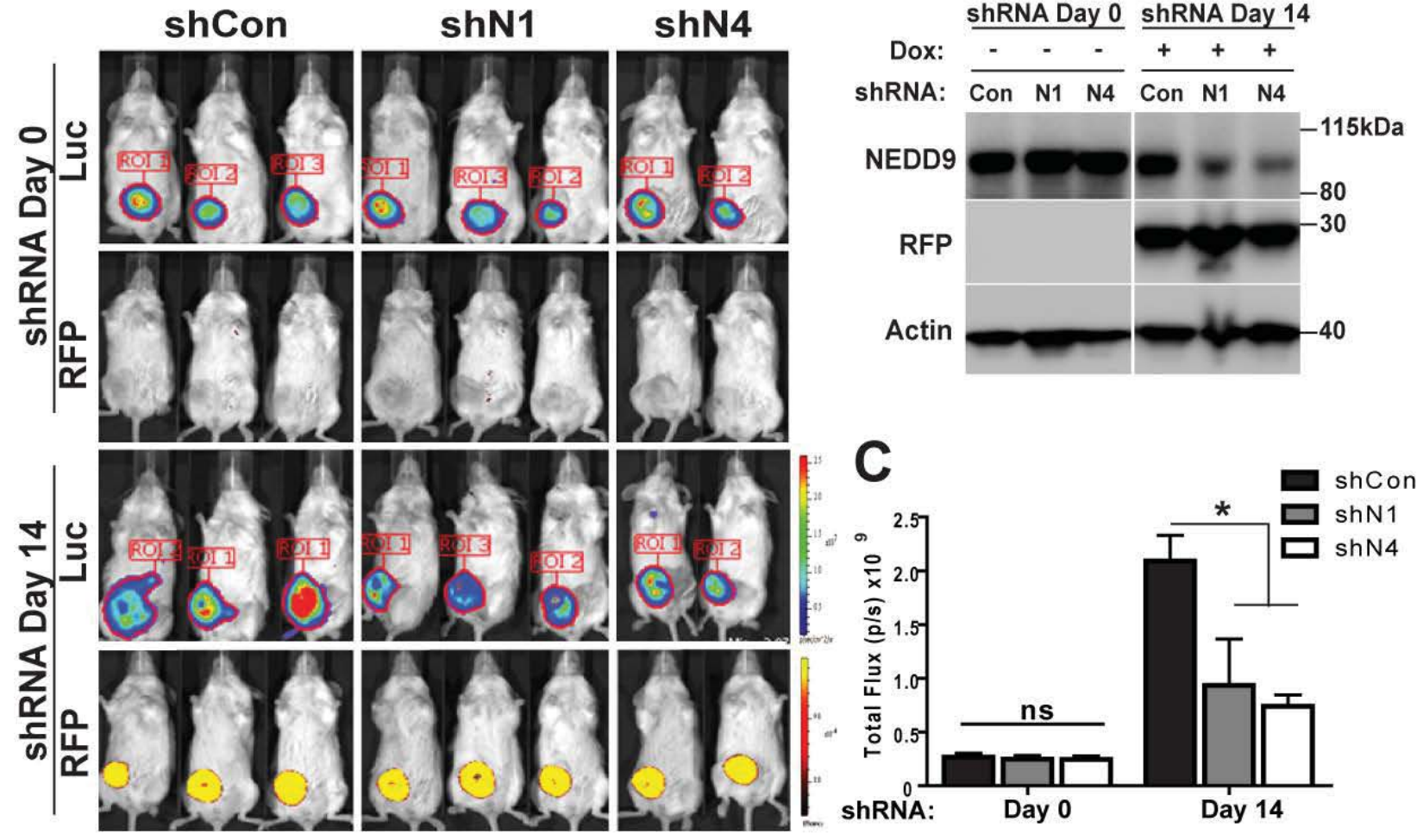

B

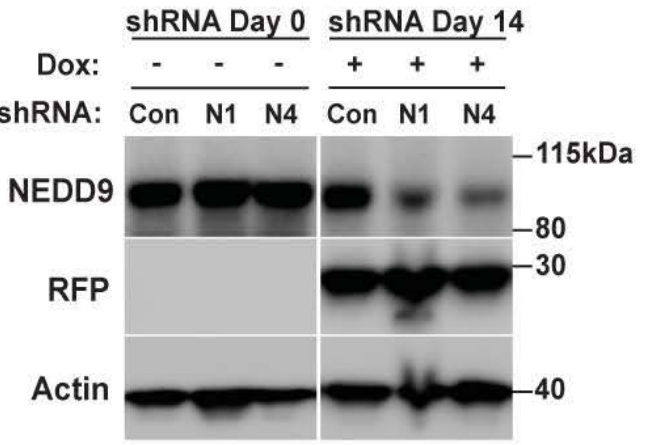

D

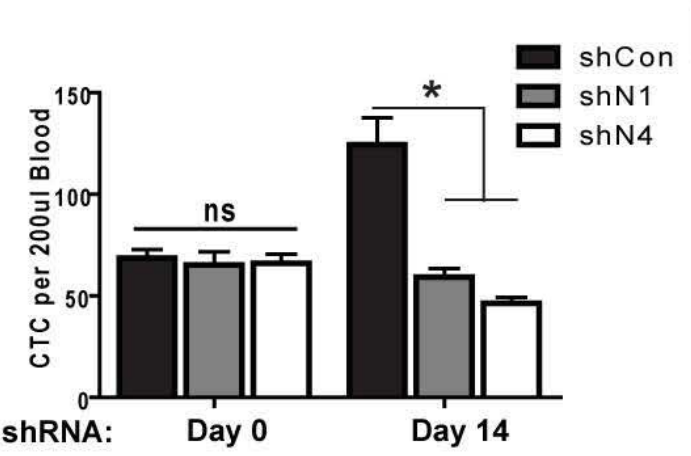

$\mathbf{F}$

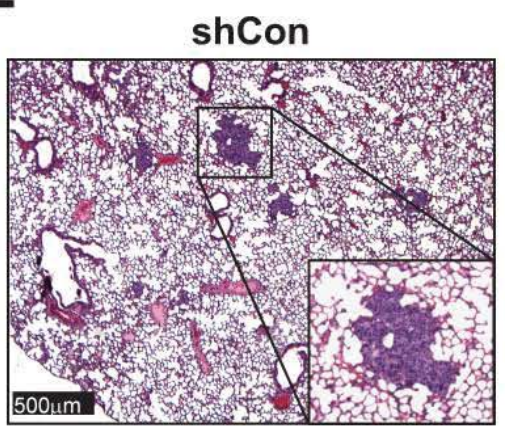

E

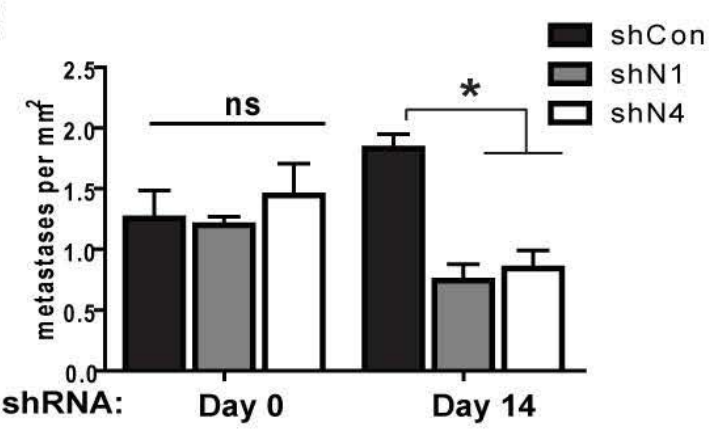

G
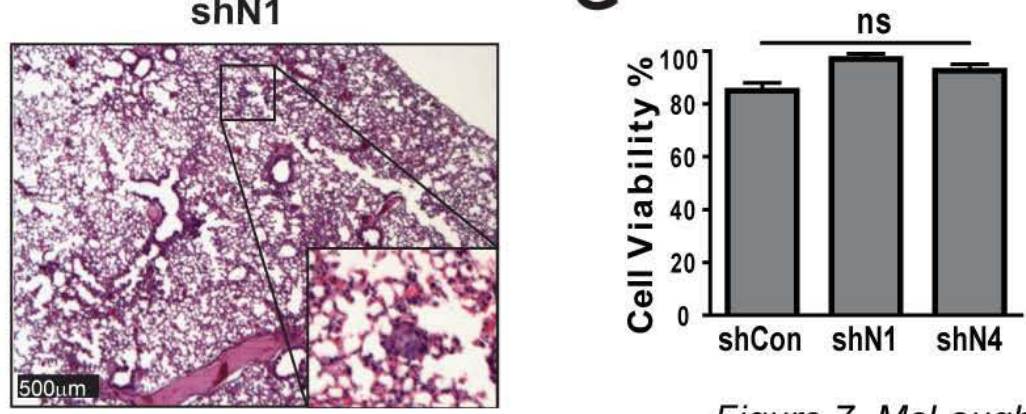

Figure 7, McLaughlin 
A

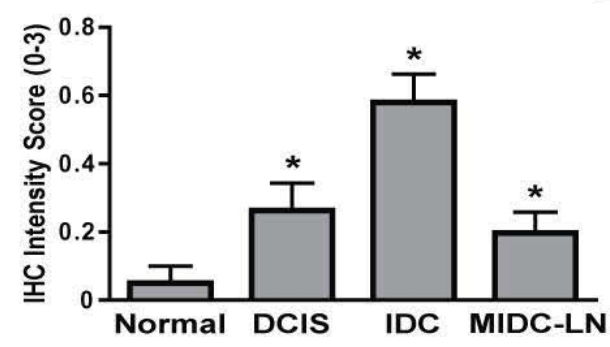

C
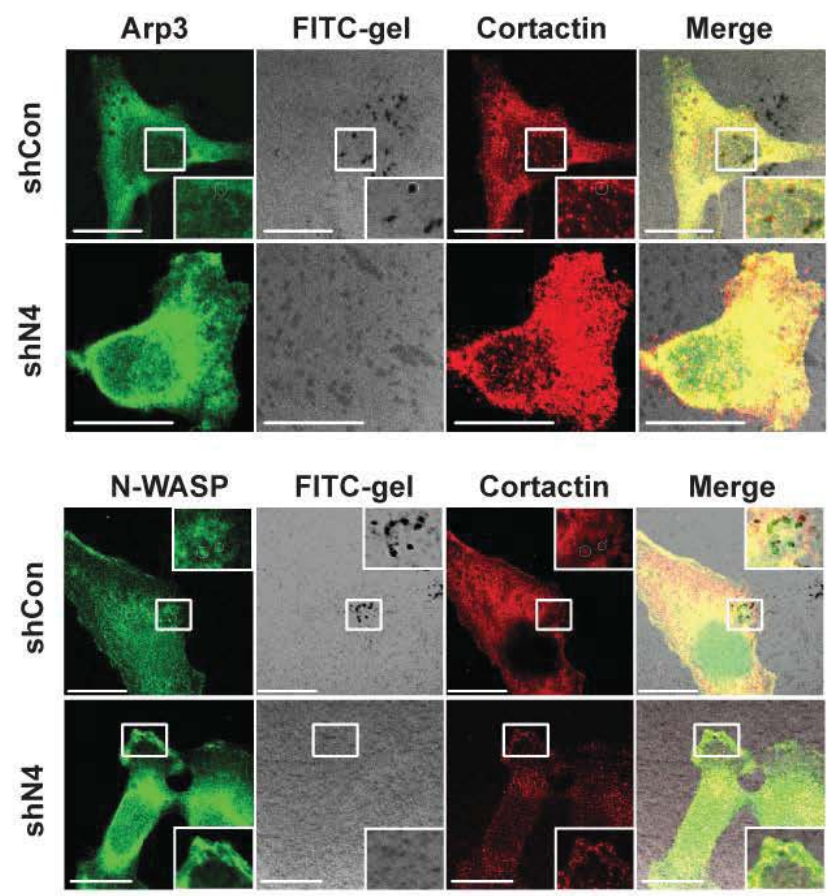

ZR-75-1

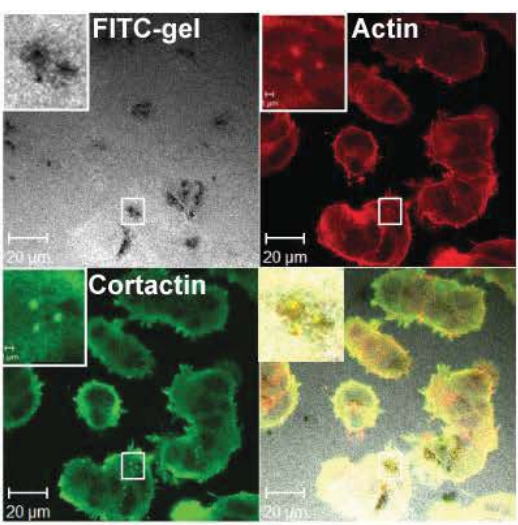




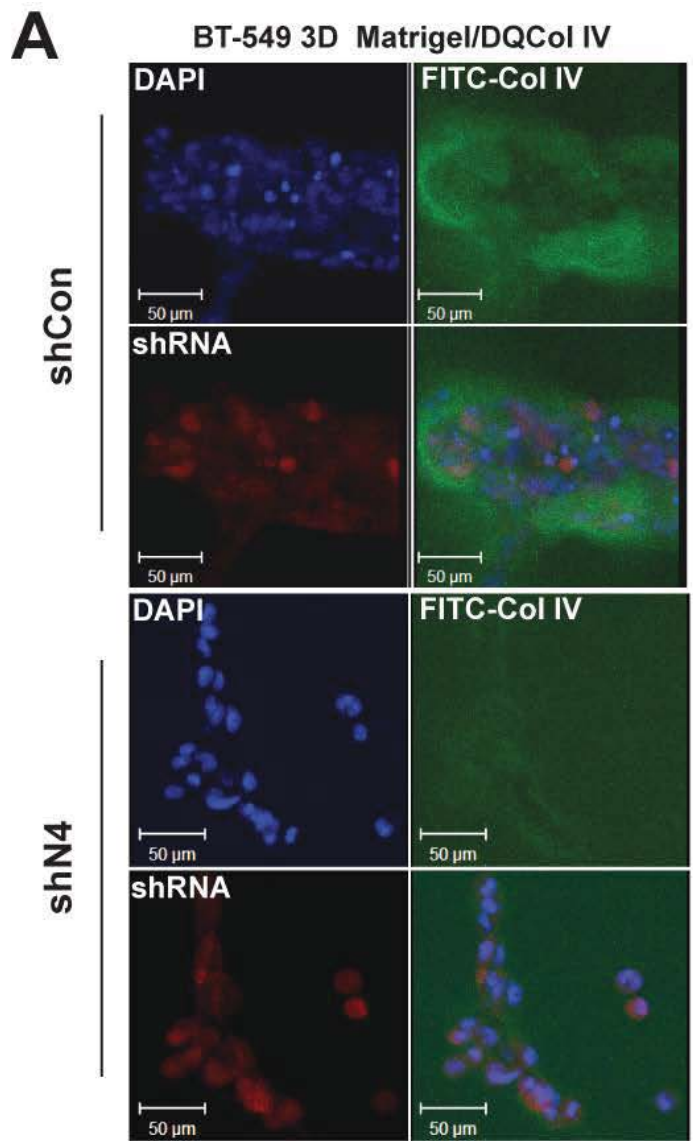

B

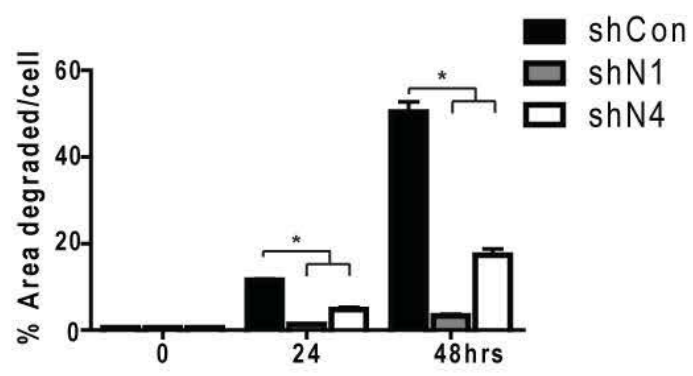

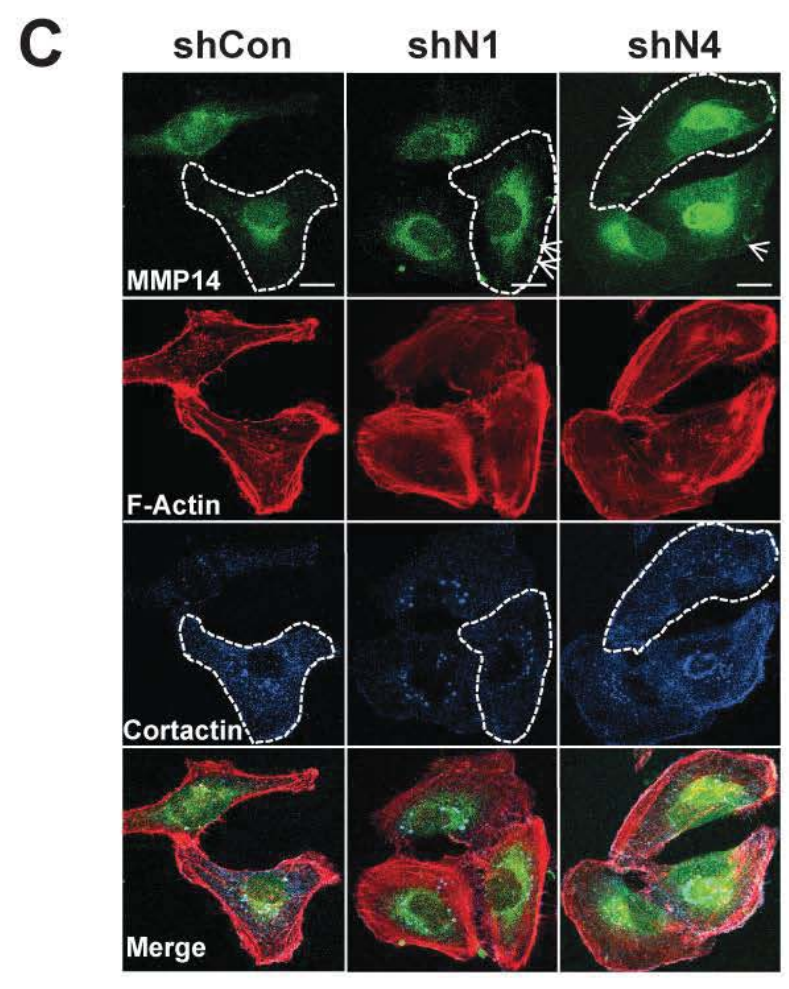

Supplementary Figure 2, McLaughlin 

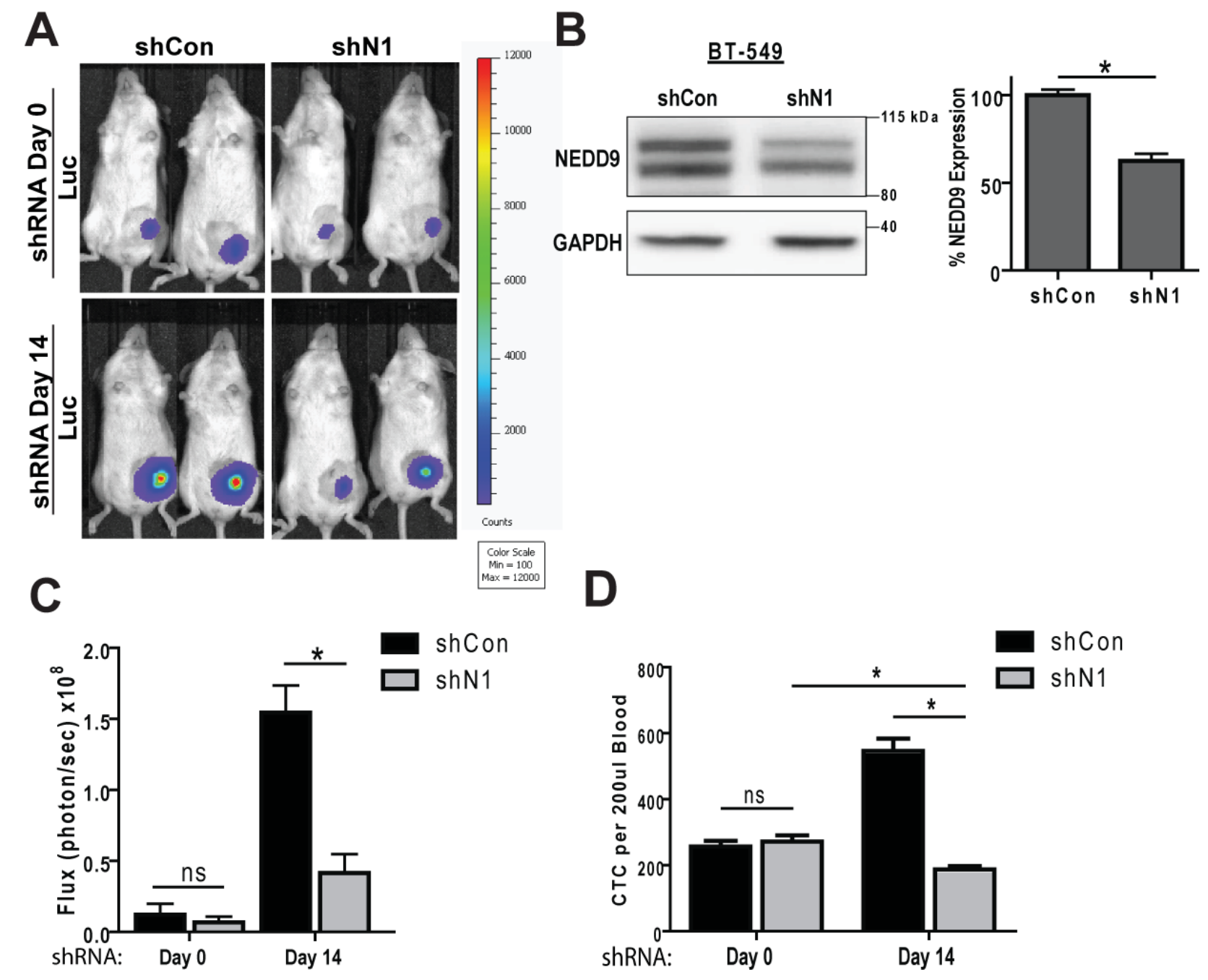
Study 3: Generation of a Mammary Specific Nedd9 Knock-in Mouse Model

Ryan J. Ice and Elena N. Pugacheva 


\begin{abstract}
NEDD9 protein levels are often increased in many types of cancers and are an established indicator of poor prognosis. Breast cancer patient samples containing high levels of NEDD9 correlate strongly with a more invasive and metastatic phenotype. Additionally, in breast cancer cell lines, NEDD9 plays a critical role in the formation of active invadopodia and increased migration. In order to investigate the impact of NEDD9 overexpression in vivo, we generated a transgenic mouse model with an extra copy of inducible human NEDD9 cDNA inserted into the ROSA26 locus on chromosome 6 , which when crossed with any tissue specific Cre recombinase expressing mouse strain, will result in overexpression of NEDD9. To assess the specific role of NEDD9 overexpression in mammary tumorigenesis and mammary gland development, we crossed the founder line with a mouse strain expressing Cre recombinase under the control of the mouse mammary tumor virus promoter, MMTV-Cre. The resulting line overexpresses NEDD9 protein specifically in the mammary gland epithelial tissue. The MMTVCre-NEDD $9^{+/+}$mice were further crossed with the MMTV-Her2 line to mimic oncogene-driven tumorigenesis often seen in Her2 overexpressing patients in human breast cancer. The control MMTV-Cre, MMTV-Cre/NEDD $9^{+/+}$and experimental MMTV-Her2 and MMTVCre/NEDD $9^{+/+} /$Her2 lines were successfully produced and further studies will be needed to examine tumor initiation and progression. The characterization of mammary gland development in founder MMTV-Cre- NEDD9 line was completed and showed a significant increase in the branch to bud ratio indicating a possible increase in invasion potential during mammary gland development. Taken together the generation of a transgenic mammary gland specific NEDD9 overexpressing model will aid in uncovering the impact of NEDD9 on tumor initiation and progression.
\end{abstract}




\section{Introduction}

Invasive breast cancer remains a difficult to treat disease effecting one in eight women during their lifetime, often with poor prognosis when metastases are present at the time of diagnosis (Siegel 2012, Rugo 2008). Although mutations in tumor suppressor genes such as p53 are oncogenic, it is common that increases in signaling not due to mutation are found in pro-metastatic and pro-survival signaling proteins such as: Src, FAK, and Akt (Dimri 2007, Lahlou 2007, Maroulakou 2007). NEDD9, a scaffolding protein commonly overexpressed in various tumor types including breast, is known to enhance the activity of such pathways leading to increased invasion, migration, and survival resulting in poor prognosis (Izumchenko 2009).

In order to dissect the role of NEDD9 protein in mammary tumorigenesis in vivo it is imperative to define first if overexpression of NEDD9 affects normal mammary epithelium function. Additionally, the role of NEDD9 in mammary gland development is an area that has not yet been explored. Since NEDD9 has been shown to increase the activity of MMPs and degradation in human tumors, and mammary gland bud formation has been shown to be an MMP dependent process it would stand to reason that NEDD9 may play a role in mammary gland development (McLaughlin 2013, Fata 2004).

We adopted the strategy of overexpressing human NEDD9 in the mammary gland alone instead of the entire mouse since NEDD9 has been reported the play a role in Alzheimer disease and necessary cilia functions in the gonads important for fertility (Zhang 2006). To date, there are no known tumorigenic mutations of NEDD9, however NEDD9 is commonly overexpressed and is known to be involved in tumorigenesis. The importance of NEDD9 in mammary tumorigenesis has been illustrated previously by knocking out Nedd9, in the context of an oncogene driven spontaneous tumor model system (Izumchenko 2009). Nedd9 knockout in the context of MMTV-PyVmT and MMTV-neu mouse mammary tumor models showed a decrease in the incidence, increased latency, reduced tumor size and reduced number of metastasis when compared to original MMTV-PyVmT mice.

When crossed with MMTV-Her2, Nedd9 knock out animals show a difference of $56 \%$ in the number of mice that form tumors within one year of age. It should be noted however that the Cas family member, p130Cas does have the ability in many instances to compensate for the loss of Nedd9. We believe our novel model system more accurately recapitulates patient tumors, since patients present with increased levels of NEDD9 protein, and that p130Cas 
compensation is not a factor in overexpression we feel our model to be more clinically relevant and possess the ability to test NEDD9 targeted therapies.

The purpose of this study is to evaluate the role of NEDD9 overexpression in the development of mammary gland tumors in the physiologically relevant context. Wild type human NEDD9 will be specifically expressed only in the mammary gland epithelia via the MMTV promoter. Upon confirmation of specific and significant expression of NEDD9 (recapitulating the expression of NEDD9 in human tumors) in mammary epithelium this strain will be crossed with a well characterized mouse model of mammary tumorigenesis, MMTV-Her2. The tumor initiation and progression in this strain is driven by overexpression of Her2/ErbB2, a growth factor receptor of the EGFR family, leading to spontaneous mammary gland tumors and metastasis, seen in human cancers characterized by Her2 overexpression.

\section{Materials and Methods}

Generation of conditional NEDD9 knock IN (Floxed-STOP-NEDD9) transgenic strain and locus insertion.

A targeting vector was utilized for homologous recombination at the Rosa26 locus of the murine genome. The insertion portion of the targeting vector contains a CAAG ubiquitously expressed promoter (CMV immediate early enhancer/ chicken b-actin promoter fusion), coupled to a start site, followed by a stop codon in frame, flanked by LoxP Cre recombinase recognition cassettes. Downstream of the LoxP cassette is the coding sequence for NEDD9 (Fig.1A). Vector design, mouse embryo injection, and transplantation was performed by GenOway, Inc. into a C57BL/6 background. The PCR screening for homologous recombination at the 5' end of the targeting vector was performed using set of designed primers: GX6043/44, generating $1870 \mathrm{~kb}$ fragment (Fig.1B). Once chimera mice were generated yielding homozygous floxed NEDD9 knock-in offspring, embryos were cryopreserved and stored at the Jackson Laboratory.

Due to the significant impact of genetic background on tumorigenesis the NEDD9 transgene was transferred to FVB background using speed congenic technology executed by Charles Rivers Laboratory and WVU Transgenic Animal Facility. The successful transfer of NEDD9 transgene was confirmed by PCR analysis as outlined below.

\section{Backcrossing strategy}

Floxed-STOP-NEDD9 mice were backcrossed from a C57BL/6J background into an FVB/J strain background via the selection using MaxBax (Charles Rivers) single nucleotide 
polymorphism analysis of the offspring. By comparing unique single nucleotide polymorphisms possessed by either C57BL/6 or FVB/J we were able to determine which mice to use for further breeding in order to achieve the greater than $96 \%$ of $\mathrm{FVB} / \mathrm{J}$ mice background still containing the Cre-NEDD9 knock-in allele. The successful transfer was produced after the 3rd crossing (N3) with the $98 \%$ of transfer to FVB genetic background, resulting in FVB/J-Floxed-STOP-NEDD9. Similar genotyping protocol as for C57BL/6J was used to confirm the presence of NEDD9 transgene in FVB/J strain (Fig.2A).

\section{Generation of FVB/J-MMTV-Cre-NEDD $9^{+/+}$mice for mammary gland specific upregulation of NEDD9.}

The FVB/J-Floxed-STOP-NEDD9 mice were crossed with FVB/J-MMTV-Cre mice expressing Cre recombinase under the mouse mammary tumor virus (MMTV) promoter, specific for mammary gland epithelium. This line was obtained via a gracious gift from Dr. J Michael Ruppert. The resultant offspring were genotyped using set of designed primers to detect wild type, recombined and Cre-excised allele of NEDD9. Homozygous for NEDD9 insertion FVB/JMMTV-Cre/NEDD $9^{+/+}$mice were used for analysis of normal mammary gland development and for further crosses with mammary tumor models. The upregulation of NEDD9 in mammary epithelium was confirmed by western blot analysis (Fig. 2C).

\section{Generation of FVB/J-MMTV-Cre-NEDD ${ }^{+/+}$-Her2 mice for mammary gland specific upregulation of NEDD9 and Her2/Erbb2/neu oncogene.}

FVB/J-MMTV-Cre/NEDD $9^{+/+}$mice were crossed with FVB/J-MMTV-Her2/Erbb2/neu mouse model for mammary tumors in humans purchased from Jackson Laboratories. This strain expresses unactivated wild type cDNA of rat Erbb2. The resultant strain expressing all three transgenes: Cre, NEDD9 and Her2 was confirmed by PCR analysis using target specific primers as outline in the Genotyping section. This line along with MMTV-Cre, and MMTV-Cre/NEDD9 ${ }^{+/+}$ and MMTV-Her2 are observed for the tumor formation. According to the literature MMTV-Her2 mice start forming tumors at 8 months or older. We expect that overexpression of NEDD9 will promote tumorigenesis by shortening the tumor initiation period, including earlier tumor cell invasion and metastasis resulting in a faster growing and more aggressive cancers.

\section{Genotyping}

Mouse DNA was isolated from $0.3-0.5 \mathrm{~cm}$ tail fragment using the PrepEase kit (Affymetrix) as per manufactures instructions. Mice were genotyped using the following primers: human 
NEDD9 knock-in allele $=$ 5'-TCCCAGAGTGTGCCGAGGAA-3'/5'GGGCCTTTTGCTGATGAGGG-3', Rosa26=5'-CAATACCTTTCTGGGAGTTCTCTGC-3'/5'CTGCATAAAACCCCAGATGACTACC-3', Her2=5'-CGgAACCCACATCAGGCC -3'/5'TTTCCTGCAGCAGCCTACGC and Cre= 5'-GGTTCTGATCTGAGCTCTGAGTG-3'/5'CATCACTCGTTGCATCGACCG-3'. Using the previously listed primers and PCR Ranger mixture (Bioline) resulted in the following band sizes, indication the presence of the allele: NEDD9 knock-in= 553 base pairs, Rosa26 300 base pairs, and Cre $=900$ base pairs, Her2= 600 base pairs (Fig 2A).

\section{Generation of MEFs and knock-in allele test}

Mouse embryonic fibroblasts, MEFs, were generated from embryos harvested at day 12 (E12). Sterile technique was used to avoid cross contamination. Collected embryos were minced using \#10 scalpel blades and incubated for 15 minutes in $0.25 \%$ Trypsiin/EDTA at $37^{\circ} \mathrm{C}$. Full media, Dulbecco's modified Eagle's medium supplemented with 15\% fetal bovine serum and antibiotic-antimycotic, was added to neutralize the trypsin. Cells were pelleted by centrifugation, re-suspended in full media, plated, and passaged. Cells were cryopreserved for later use.

In order to test the effectiveness of the transgenic construct, inducible NEDD9 knock-in MEF cell lines were infected with pre-packaged adenovirus expressing GFP (green fluorescent protein), Ad-Cre-GFP (Vector Biolabs) as per manufactures instructions. Infection was monitored through fluorescent microscopy and in $24 \mathrm{~h}$ reached $100 \%$ efficacy based on the GFP fluorescence when compared to uninfected control. Control and Ad-Cre-GFP infected cells were lysed and probed by western for NEDD9 and GAPDH protein levels as previously described (Ice 2013) (Fig.2B).

\section{Mammary gland resection and analysis}

The fourth inguinal mammary gland was resected and prepared as described in (de Assis 2010). Briefly, MMTV-Cre recombinase expressing control and MMTV-Cre/NEDD9 ${ }^{+/+}$ knock-in virgin female mice were euthanized at 6 weeks of age and mammary glands resected. Mammary glands were placed on glass slides and allowed to dry for 10 minutes. Slides were then places in Carnoy's fixative (75\% Acetic acid, 25\% ethanol) for two days. Slides were then rinsed in $70 \%$ ethanol for one hour, followed by distilled water for 30 minutes. Mammary glands were then stained in Carmine Alum stain $(0.2 \%$ carmine dye, $0.5 \%$ aluminum potassium sulfate in distilled water) for two days. Ethanol washes were then performed for 30 minutes each in 
increasing concentrations of ethanol $(70 \%, 80 \%, 95 \%, 100 \%)$. Tissue was then allowed to delipidate in xylene for two days followed by mounting. Whole mounts of mammary glands were imaged at $4 \mathrm{X}$ using an Olympus AZ70 microscope; after which bud to branch ratio was calculated in 5 mice per group. Statistical software (Graphpad Prism) was used to determine significance via Students T-test with a p-value of 0.0324 . 


\section{$\underline{\text { Results }}$}

\section{Experimental rationale and generation of FVB/J-Floxed-STOP-NEDD9 mice}

To determine the role of NEDD9 in tumorigenesis and metastasis in vivo, we developed a transgenic mouse strain which, when in the presence of Cre recombinase, overexpresses NEDD9. Since we do not predict abundant NEDD9 to be oncogenic alone, we crossed our strain with the oncogenic strain MMTV-Her2. The generated mice containing all three transgenes, Cre, NEDD9, and Her2, will be evaluated for tumor latency, burden, size, and metastasis. An additional advantage of the Cre inducible model design is the ability to use the multitude of tissue and time specific Cre recombinase promoter strains available.

\section{Evaluation of Cre-dependent expression of NEDD9 in FVB/J-Floxed-STOP-NEDD9}

Through utilizing mouse embryo fibroblasts generated from FVB/J-Floxed-STOP-NEDD9 mice, we were able to overexpress NEDD9 upon infection with adenoviral Cre recombinase. Using western blot analysis we detected a robust overexpression of NEDD9 when compared to uninfected MEFs (Fig. 2B); therefore, indicating the Cre recombinase inducible vector is functional and producing a NEDD9 product of full length which is recognized by monoclonal antibody specific to NEDD9. Mammary glands harvested from MMTV-Cre and MMTVCre/NEDD $9^{+/+}$indicate that there is indeed an increase in NEDD9 in the mammary fat pad.

\section{NEDD9 overexpression increases mammary gland budding}

In order to evaluate if NEDD9 overexpression effects the normal mammary gland development we assessed the difference in mammary gland branching between MMTV-Cre and MMTV-Cre/NEDD9 knock-in virgin mice. In order to determine if there is a difference in mammary development whole mammary gland mounts were prepared from 6 weeks old virgin mice according to the protocols outlined in material and methods section. We found that upon NEDD9 overexpression there was an increase in the budding to branch ratio, indicating that NEDD9 plays a role in mammary gland development (Fig. 3A-B). Overexpression of NEDD9 either increases proliferation and/or invasion of normal mammary epithelial cells or could potentially predispose to cancer. Further work is needed to validate this phenotype, but it is 
important to take it into account when analyzing the NEDD9 overexpression impact on the background of Her2 oncogene. The currently ongoing characterization of FVB/J-MMTVCre/NEDD $9^{+/+} /$Her2 mice will allow us to define the role of NEDD9 in mammary tumorigenesis. 


\section{Discussion}

Cre recombinase inducible transgenic mouse model systems provide a versatile tool for the study of protein in either a tissue or time specific manner. By developing a Cre inducible human NEDD9 knock-in mouse model system we are able to study the role of overexpressed NEDD9 in a mammary specific context similar to what is present in breast cancer patient samples.

The previous studies have investigated the role of NEDD9 in mammary tumorigenesis (Izumchenko 2009, Little 2012) using NEDD9 knockout mouse model crossed with mammary specific oncogenic mouse strains, MMTV-PyVmT and MMTV-Her2. While both studies did show that NEDD9 plays a role in tumor growth, latency, and burden. A major complication of this strategy is the ability of p130Cas, a protein in the same family as NEDD9, to be induced and compensate for loss of NEDD9. In this instance the importance of NEDD9 in tumorigenesis would be difficult to accurately evaluate understate. It is for this reason that we believe our developed model system more accurately portrays native mammary tumorigenesis.

Both NEDD9 and Her2 are found to be overexpressed in breast cancer, and therefore make a more clinically relevant model system to study tumorigenesis. By creating this model system we will be able to study novel anti-metastatic therapies, since NEDD9 has been shown to be a marker of metastatic disease. Additionally, the inducible nature of our model provides versatility through crossing it with other tissue and time specific Cre promoter driven mouse strains. Finally, our model can be helpful for future drug development targeting NEDD9 and NEDD9-dependent signaling pathways.

Taken together our results illustrate the creation and application of a Cre recombinase inducible NEDD9 knock-in mouse model. Additionally, we were able to establish using our novel model system that overexpressing NEDD9 in mouse mammary glands increases mammary gland development, which could be attributed to the role of NEDD9 in increasing MMP activity during tumorigenesis (McLaughlin 2013), although more studies need to be performed to prove this point directly. 


\section{References}

de Assis S, Warri A, Cruz MI, Hilakivi-Clarke L. "Changes in mammary gland morphology and breast cancer risk in rats." J Vis Exp. 2010 Oct 16;(44).

Dimri M, Naramura M, Duan L, Chen J, Ortega-Cava C, Chen G, Goswami R, Fernandes N, Gao Q, Dimri GP, Band V, Band H. "Modeling breast cancer-associated c-Src and EGFR overexpression in human MECs: c-Src and EGFR cooperatively promote aberrant three-dimensional acinar structure and invasive behavior." Cancer Res. 2007 May $1 ; 67(9): 4164-72$.

Fata JE, Werb Z, Bissell MJ. "Regulation of mammary gland branching morphogenesis by the extracellular matrix and its remodeling enzymes." Breast Cancer Res. 2004;6(1):1-11.

Izumchenko E, Singh MK, Plotnikova OV, Tikhmyanova N, Little JL, Serebriiskii IG, Seo S, Kurokawa M, Egleston BL, Klein-Szanto A, Pugacheva EN, Hardy RR, Wolfson M, Connolly DC, Golemis EA. "NEDD9 promotes oncogenic signaling in mammary tumor development." Cancer Res. 2009 Sep 15;69(18):7198-206.

Lahlou H, Sanguin-Gendreau V, Zuo D, Cardiff RD, McLean GW, Frame MC, Muller WJ. "Mammary epithelial-specific disruption of the focal adhesion kinase blocks mammary tumor progression." Proc Natl Acad Sci U S A. 2007 Dec 18;104(51):20302-7.

Maroulakou IG, Oemler W, Naber SP, Tsichlis PN. "Akt1 ablation inhibits, whereas Akt2 ablation accelerates, the development of mammary adenocarcinomas in mouse mammary tumor virus (MMTV)-ErbB2/neu and MMTV-polyoma middle T transgenic mice." Cancer Res 2007; 67: 167-77.

McLaughlin SL, Ice RJ, Rajulapati A, Kozyulina PY, Livengood RH, Kozyreva VK, Loskutov YV, Culp MV, Weed SA, Ivanov AV, Pugacheva EN. "NEDD9 Depletion Leads to MMP14 Inactivation by TIMP2 and Prevents Invasion and Metastasis." Mol Cancer Res. 2014 Jan;12(1):69-81.

Rugo HS. "The importance of distant metastases in hormone-sensitive breast cancer." Breast 2008; 17 Suppl 1: S3-8.

Siegel R, DeSantis C, Virgo K, Stein K, Mariotto A, Smith T, Cooper D, Gansler T, Lerro C, Fedewa S, Lin C, Leach C, Cannady RS, Cho H, Scoppa S, Hachey M, Kirch R, Jemal A, Ward E. "Cancer treatment and survivorship statistics, 2012." CA Cancer J Clin. 2012 Jul-Aug;62(4):220-41.

Zhang Z, Kostetskii I, Tang W, Haig-Ladewig L, Sapiro R, Wei Z, Patel AM, Bennett J, Gerton GL, Moss SB, Radice GL, Strauss JF 3rd. "Deficiency of SPAG16L causes male infertility associated with impaired sperm motility." Biol Reprod. 2006 Apr;74(4):751-9. 


\section{FIGURES:}

A

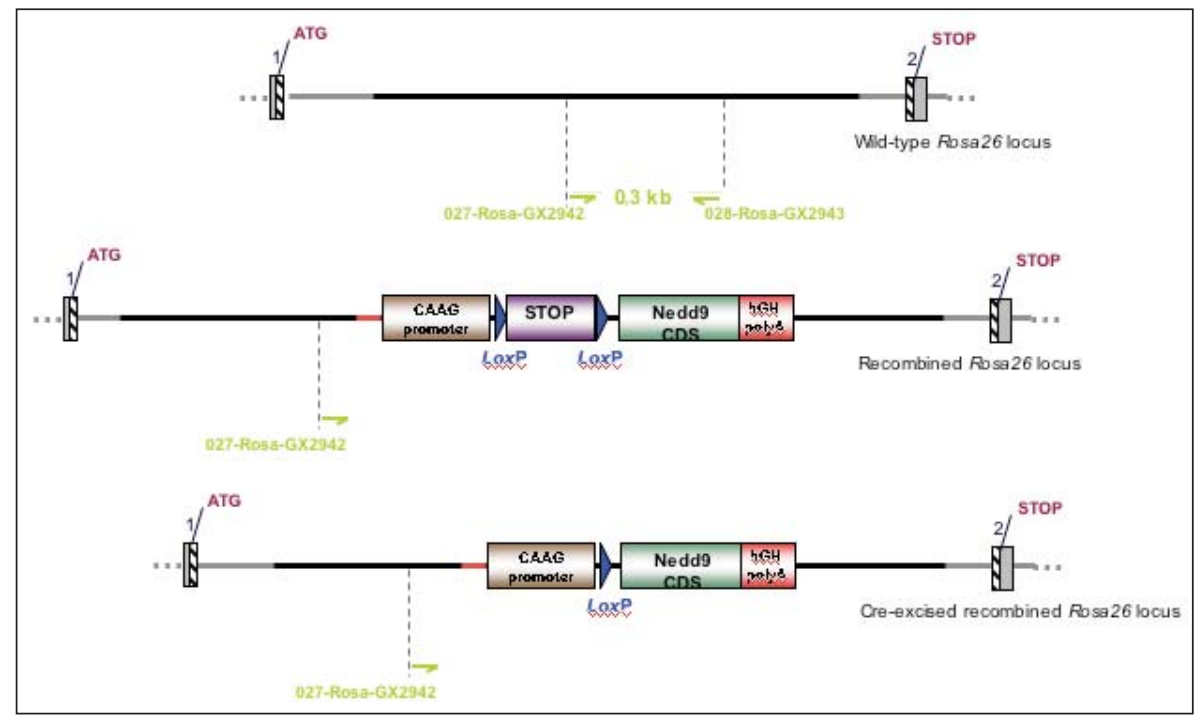

B

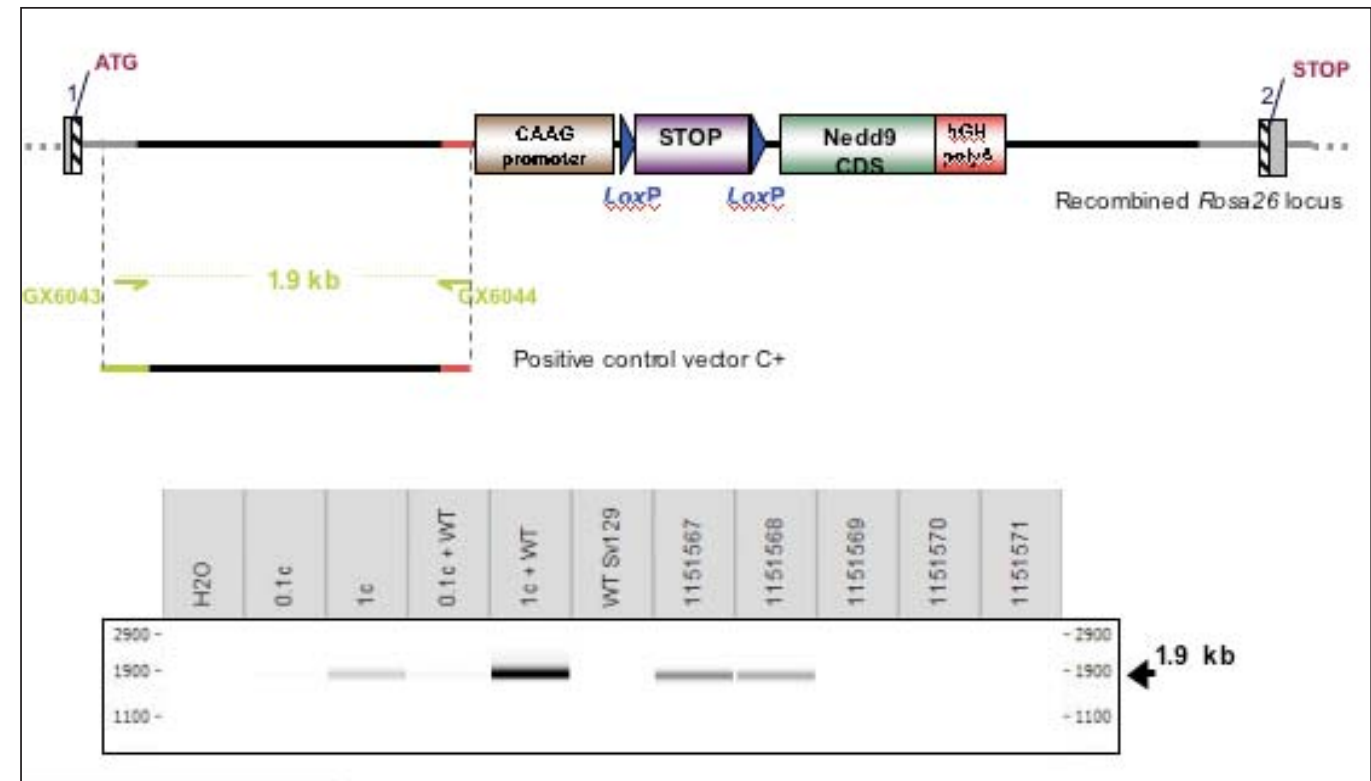

Figure 1. Design and expression of Cre inducible nedd9 knock-in. A) Design of Cre recombinase inducible NEDD9 knock-in vector for homologous recombination at the Rosa26 locus (top). In the presence of Cre recombinase the STOP cassette is resected out of the genome due to flanking LoxP sites (middle) resulting in CAAG promoter driven NEDD9 expression (bottom) B) PCR test using GX primer set for homologous recombination. 


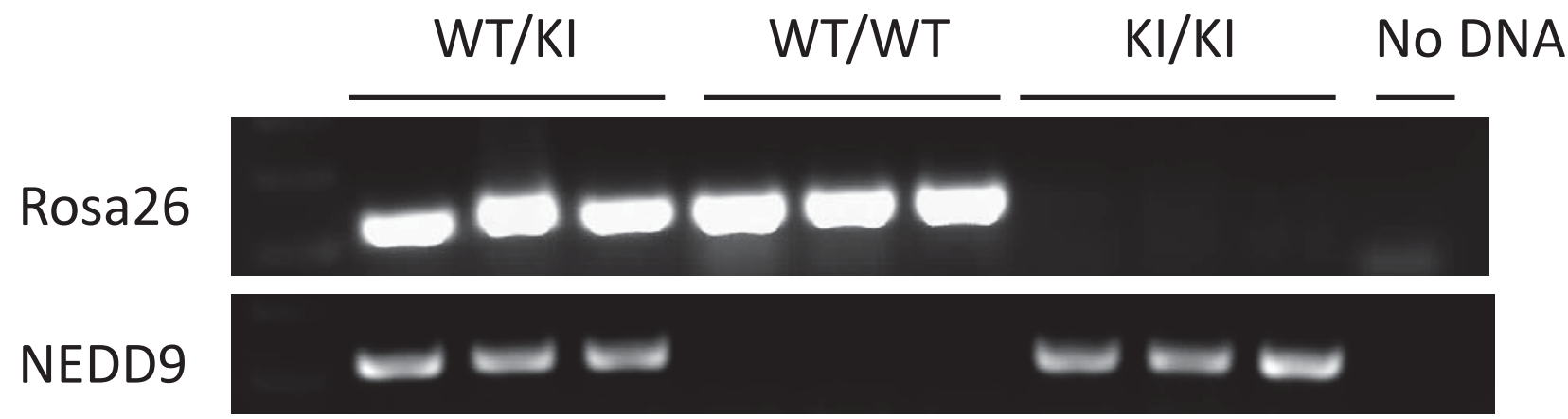

B

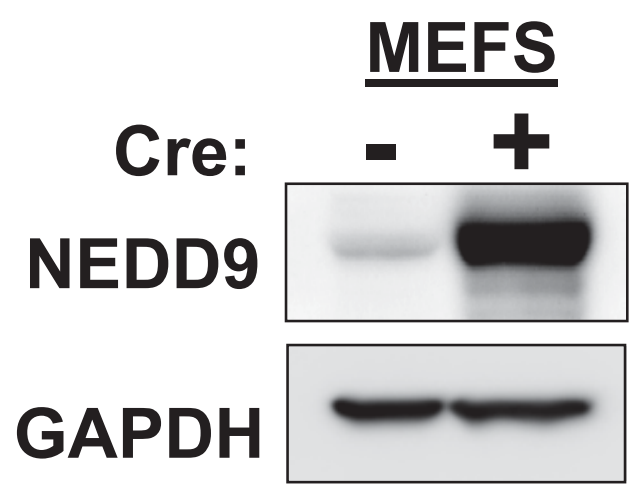

C

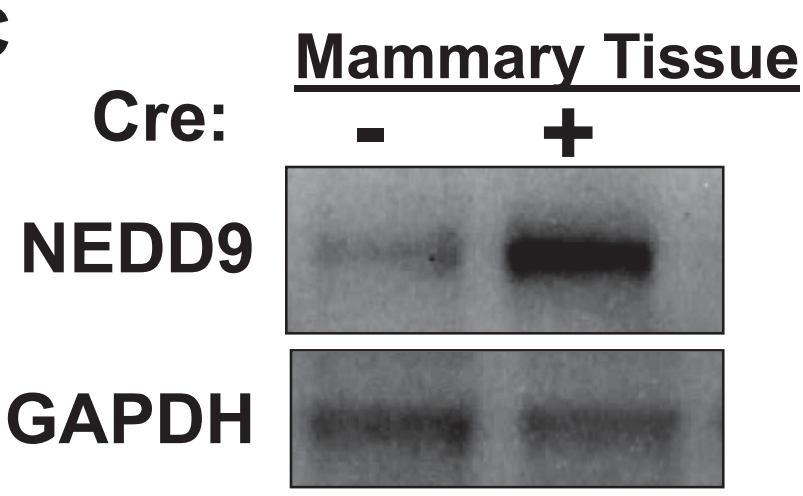

Figure 2. A )Genotyping results in triplicate indicating the presence of either the Rosa26 loci or the NEDD9 loci. B) Western blot analysis of lysates of mouse embryo fibroblasts prepared from nedd9 knock-in mice with and without being infected with adenoviral Cre recombinase. NEDD9 protein levels are greatly increased and producing full length transcript upon infection. C) Western blot analysis of mammary tissue taken from Cre-Nedd9 and Cre-WT mice. Levels of NEDD9 protein are increased in the Cre positive mouse. 


\section{A}
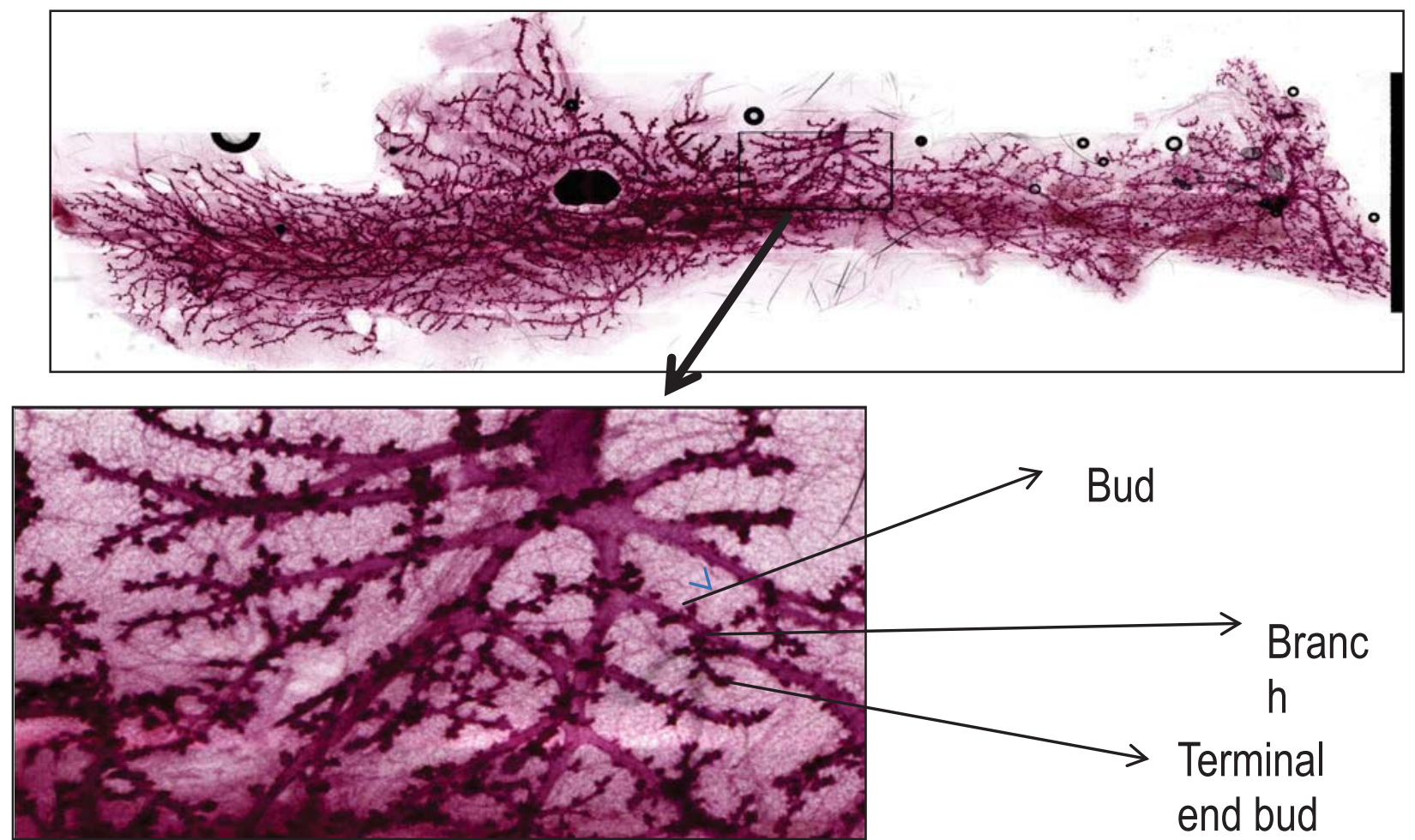

B

\section{Virgin Adult Mammary Pad}

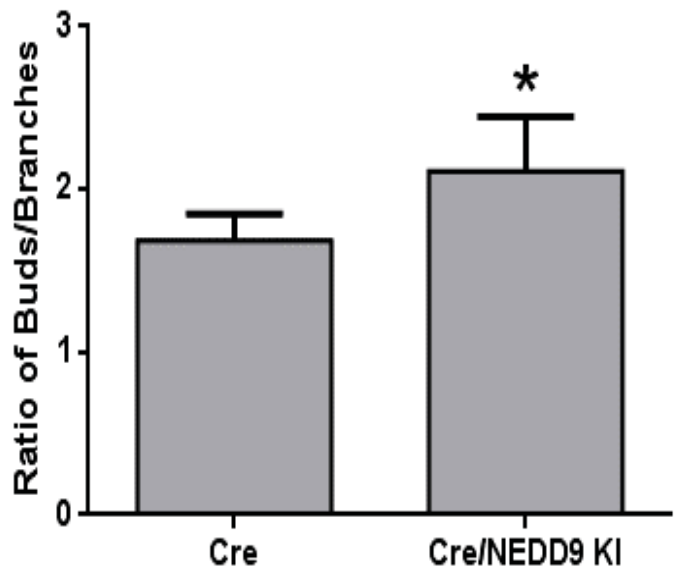

Figure 3. NEDD9 overexpression results in increased budding of mammary glands. A) Top, a representative image taken of a whole mount section of the fourth inguinal mammary gland of a 6 week old virgin Cre/NEDD9 knock-in mouse. Bottom, a closer view of a mammary tree. Arrows indicate a bud, branch, and terminal end bud. B) Quantitation of bud to branch ratio indicating a significant increase in mammary gland development upon NEDD9 overexpression. The $n=5$ with a $p$-value of 0.0324 . 
Study 4: Development of breast cancer patient derived xenograft mouse model for the testing of AURKA inhibitor sensitivity and biomarkers.

Ryan Ice and Elena Pugacheva 


\section{Abstract}

The ability to mimic tumor development and metastasis in mouse models in vivo is critical for the development of novel targeted therapies. Unfortunately, genetically engineered mouse models (GEMs) which produce tumors through the induction of a single oncogene or elimination of a single tumor suppressor are rarely seen in the human population. Most tumors are produced through sequential induction/mutation or elimination of multiple genes and selection through the prolonged period of time. Additionally, models for hormone related cancers, such as breast and prostate cancer, do not recapitulate the human tumor due to differences in hormone production/regulation cycles between mouse and human physiology. Cell line derived xenograft mouse models are also fraught with obstacles, such as: homogeneity, aberrant mutations due to being grown in culture, and lack of tumor microenvironment. Often cell line derived xenografts fail to recapitulate patient-specific pattern of metastatic spread. Due to these limitations, patient derived xenografts have been developed and are becoming a widely used tool for cancer therapeutic research. In our study we establish the patient derived breast cancer xenografts (PDBCX). These will be later used to define the efficacy of AURKA inhibitor (alisertib-MLN8237) in the treatment/or prevention of metastasis to the local and distant organs and could be used to assess tumor susceptibility and identify biomarkers of drug response to streamline the eradication of metastases. 


\section{Introduction}

Breast cancer remains the most common non-skin cancer among women and is considered the second leading cause of cancer related mortality in women. The vast majority of deaths are caused by metastatic disease. Despite significant progress in treatment options, a cure of metastatic breast cancer remains elusive. Therefore, it is clear that novel treatment strategies are required to be developed. A focus on invasion and metastasis is important since as breast cancer advances from regional to metastatic disease survival rates drop from 82 percent to 26 percent (Siegel 2012). Unfortunately, the translation from cancer cell biology to clinical application has been fraught with peril. One study suggests that as many as 90 percent of new cancer drugs fail in the clinic (Hait 2010). One such reason for the low rate of success is the lack of accurate in vivo models of breast cancer. Current models utilize cell lines which have been selected for proliferation and for the ability to grow on non-physiological surfaces, such as plastic. Moreover, when breast cancer cell lines are utilized for xenograft injections the tumor microenvironment does not recapitulate the endogenous tumor microenvironment. The influence of the tumor microenvironment has become understood and is necessary for accurate models for inhibitor testing. Under these circumstances, it is understandable that data generated using such tools may not be directly translatable to clinical applications (Clarke 2009, Voskoglou-Nomikos 2003).

By developing the ability to engraft tumor tissue taken from patients and engraft orthotopically into immunocompromised mice we are able to retain both the tumor heterogeneousness and the surrounding tumor microenvironment of the original tumor. Previous studies have shown that through the use of patient derived xenograft transplantation one can more accurately predict the response of patients to various inhibitors, allowing for multiple treatment regiments to be tested simultaneously (DeRose 2011). Additionally, patient derived xenografts are able to phenocopy the location of metastasis presented by patients, while cell line generated tumors do not, illustrating there utility in studying inhibitors that target metastasis (Press 2008, Carey 2010, Daniel 2009).

Aurora kinase $A(A U R K A)$ is a serine/threonine kinase which plays a key role in the G2/M transition of mitotic cells. AURKA is essential for normal mitotic spindle formation, centrosome maturation, and subsequent separation. Many cancer types, including breast, were found to have high levels of AURKA. 
AURKA inhibitors have recently entered Phase III clinical trials for cancer treatment with favorable pharmacodynamics and pharmacokinetics, and limited toxicities in hematological cancers. Unfortunately, the average benefit derived from AURKA inhibitors in advanced solid tumors has been disease stabilization with few documented instances of tumor regression (Siegel 2012, Hait 2012, Clarke 2009, Voskoglou-Nomikos 2003, DeRose 2011, Press 2008). Our understanding of the molecular factors influencing sensitivity and resistance to AURKA inhibitors remains limited. AURKA expression is directly linked to advanced tumor stage and the occurrence of metastasis (Voskoglou-Nomikos 2003). Interestingly, it was noted that the disease relapse and the metastatic dissemination was lower in those patients treated with AURKA inhibitors (alisertib). There is a critical need to determine the efficacy of AURKA inhibitors in the prevention and/or treatment of metastasis using more physiologically relevant modes such as patient-derived metastatic tumor xenografts (DeRose 2011). Identification of a spectra of cancers sensitive to AURKA inhibitors will provide new diagnostic tools to select patients who will most likely benefit from AURKA inhibitors and new therapies to treat metastasis. Completion of these studies is expected to provide new critical knowledge regarding usage of AURKA inhibitors in the treatment of late stage, high risk of metastasis patients.

In our previous study we found the inhibitor MLN8237 (alsertib) is able to inhibit metastasis in cell line derived orthotopic xenografts and that reducing the levels of NEDD9 enhanced the anti-metastatic effect of MLN8237 (Ice 2013). The goal of this study is to establish the patient derived breast cancer xenografts (PDBCX) model system and tissue bank to be used for the future purpose of evaluating the efficacy of anti-metastasis therapies.

\section{Materials and Methods}

Patient sample selection for transplantation into mice. The following clinical characteristics of the patients will be considered at the procurement stage: large tumor size $(20>\mathrm{mm})$, and stage III and IV with local/distant metastasis confirmed. Samples were either acquired from the lab of Dr. Alana Welm at the Huntsman Cancer Institute at the Univeristy of Utah or through the Cooperative Human Tissue Network at the National Cancer Institute. The number of samples for this study is based on published records and tumor uptake rates. We have collect 13 biopsies for stages III-IV (Table 1). Engraftment of late-stage tumors (stage III-IV) is 40-60\% (DeRose 2011). The NSG mouse strain has demonstrated up to $100 \%$ tumor retention and is considered the best model for human cancer transplantation (Ishikawa 2005). Based on this evidence we expect at least $60 \%$ of collected breast cancer biopsies will be successfully grafted in NSG mice. We have currently six tumors successfully engrafted. 


\section{Patient Tumor collection and processing}

Upon resection, tissue samples were collected and submerged in Dulbecco's Modified Eagle Medium, (DMEM, Sigma) and stored at $4^{\circ} \mathrm{C}$ on ice during shipment. Tissue samples were processed within 24 hours of collection. Upon arrival, samples were divided into approximately $2 \mathrm{~mm}$ cubed pieces.

Portions of tissue were fixed in $\mathrm{Zn}$ based formalin fixative (Fischer Scientific) and embedded in paraffin for later analysis of stroma profile and immunohistochemical staining. Portions of the tissue were flash frozen in liquid nitrogen for analysis by western blot using antiNEDD9 2G9, anti-AURKA (Becton Dickinson), and anti-AURKA Thr288 (Cell Signaling) as previously described (Ice 2013). Remaining tissue specimen pieces were either immediately transplanted or cryopreserved in 95\%serum, $5 \%$ dimethyl sulfoxide (Sigma) and stored in liquid nitrogen.

Engraftment of the Tumor Biopsies in NSG Mice. Breast cancer fragments are obtained from patients at the time of surgery or biopsy (before neo adjuvant treatment) and grafted into the fat pad of 4-6 weeks old female NSG mice. Mice are maintained in BSL2, pathogen-free animal housing at the WVU Animal Facility. Based on our experience, tumors start growing at the graft site between 2 to 8 weeks after surgery. Each patient derived tumor, upon successful growth in the mouse, will be isolated and the tumor mass cut into $1 \times 2 \times 2 \mathrm{~mm}(\mathrm{~W} \times \mathrm{H} \times \mathrm{L})$ fragments and transplanted into the mammary fat pad of a new NSG mouse. This procedure is called passaging of tumor and could be repeated up to 10-15 times to expand the number of animals bearing the tumor of the same origin. Finally, a portion of the tissue is stored frozen in DMSOfetal bovine serum, freeze-dried in nitrogen for use in further studies, or fixed in PBS/10\% formalin for histopathological analysis.

Histology of xenografts and comparison with original patient tumors. The comparison between the histology of the original tumors and their xenografts will be established at early and later passages by a pathologist at WVU's Pathology Core Facility (Dr. Livengood) using paraffin-embedded sections and standard imunohistochemistry (IHC) staining protocols, which are currently under preparation. The histology of the original tumor is usually conserved in xenografts (DeRose 2011). The morphology of cancer cells and the stroma will be assessed in 
original tumors and in xenografted biopsies by IHC staining with specific epithelial and cell proliferation markers to confirm the stable propagation of tumor.

\section{Tumor and Estrogen implantation}

De-identified tumor tissue samples were obtained under HIPAA guidelines with informed consent. All procedures and protocols were approved by WVU IACUC and veterinary staff. The surgical procedure of tumor transplantation and preparation was performed as described in DeRose et al. (DeRose 2011). Transplantation of tumor tissue was performed using aseptic technique under sterile conditions. NOD.Cg-Prkdcscid II2rgtm1Wj//SzJ (NSG) immunodeficient mice (Jackson Laboratory, stock 5557) were anesthetized using isoflurane/oxygen mixture between $2-0.5 \%$. Prior to surgery bupivacaine and carprofen were administered subcutaneously for local and general analgesic pain relief respectively. The fourth inguinal mammary gland of female mice 4 to 6 weeks of age was resected thus removing the mammary tree. Tumor tissue was inserted in the location of the fourth inguinal mammary gland. An incision was then made between the scapulae, where an estrogen supplement pellet was placed. $7 \mathrm{~mm}$ wound clips were used to close both incision sites. Carprofen was administered once daily for three days. Mice were monitored for seven days post-surgery until wound clips were removed.

\section{Ultrasound analysis of Tumor growth and angiogenesis.}

Following tumor transplantation, tumor size and blood flow was measured via the Vevo2100 Micro-Ultrasound System. A 40 or $50 \mathrm{mHz}$ transducer was used and a 3-dimensional image (3D) was acquired with $0.051 \mathrm{~mm}$ between images. Blood flow was assessed as the Doppler signal within the tumor. Using the integrated software, the images were reconstructed to create a 3D image of the tumor at various time points. Upon establishment of growth rate and engraftment success, tumors were resected, banked, and cryopreserved for later experiments. 


\section{Results}

\section{The rates of successful engraftment of breast cancer tissue}

The collected tumor tissue from a patient biopsies and surgical resections were transplanted into NSG mice. The following categories are currently present in our collection ER positive, Her2 positive, and triple negative (Table 1).To date we have been able to successfully grow the following transplanted tumors (PEN_001, PEN_002, PEN_003, PEN_006) which is $30 \%$ of engraftment. All successful grafts so far are invasive ductal carcinomas (IDC). The following tumors were not able to grow PEN_004, PEN_005, even though other labs have successfully achieved engraftment from these samples after prolonged growth period. Differences in engraftment rates could be due to others using mesenchymal stem cells to increase angiogenesis. Also we noticed that tumors from patient undergone neo-adjuvant chemotherapy had much slower growth rates and were not successfully grafted during 8 weeks period. To date, no indicator has been established as a marker for engraftment.

\section{Analysis of engrafted tumor growth and passaging.}

Tumor volume and growth was monitored using VEVO ultrasound (Figure 1). Transplanted tumor samples were able to re-establish blood flood and induce angiogenesis as seen by Doppler ultrasound. Engraftment was achieved in various tumor types including estrogen receptor positive tumors, which need to be supplemented with estrogen in the form of slow release estrogen implanted subcutaneously. We have collected so far four tumors that have successfully engrafted.

\section{Discussion}

Even though cell line derived tumor xenografts are of great usefulness due to the easy of manipulating protein expression and infection with viral vectors, the main caveat of cell line derived model systems is that they do not accurately replicate a spontaneous tumor. The lack of heterogeneity and stroma dynamics, together with the fact that cell lines have undergone selection for fast division and the ability to grow on tissue culture dishes, is a source for divergence of response between patients and cell line model systems. By utilizing patient tumor xenografts, which have never been grown in tissue culture, we are able to better replicate the tumor of a patient. Only by recapitulating the tumor of a patient can xenograft inhibitor studies accurately predict the response of a patient to therapy. 
Since patient tissue is human and present with foreign antigens immunocompromised mice must be used in order to avoid rejection of the patient tumor tissue. One hurdle patient derived xenograft systems still need to overcome is the lack of immune system contribution, which growing evidence shows is a major contributor for tumor development and metastasis, such as tumor associated T cells and macrophages; though this can be overcome through humanization of the immune system of the transplant mouse (Motz 2013).

Despite lacking an immune system to aid in development and metastasis, by utilizing our developed patient tumor xenograft bank we will be able to probe for markers indicating metastatic location, growth, and AURKA inhibitor susceptibility. Additionally, we will be able to gather tumors that metastasize to various locations to test whether AURKA inhibitors can be used to treat specific sites of metastasis or metastasis in general. Perhaps of even greater impact is that once gathered, our tumor tissue library can be a resource for future inhibitor studies once new technologies and inhibitors are developed without the need to recruit patients for clinical trials.

\section{References}

Carey L, Winer E, Viale G, Cameron D, Gianni L. "Triple-negative breast cancer: disease entity or title of convenience?” Nat Rev Clin Oncol. 2010 Dec;7(12):683-92.

Clarke, R. "The role of preclinical animal models in breast cancer drug development." Breast Cancer Res. 11 (suppl. 3), S22 (2009).

Daniel VC, Marchionni L, Hierman JS, Rhodes JT, Devereux WL, Rudin CM, Yung R, Parmigiani G, Dorsch M, Peacock CD, Watkins DN." A primary xenograft model of smallcell lung cancer reveals irreversible changes in gene expression imposed by culture in vitro." Cancer Res. 2009 Apr 15;69(8):3364-73.

Dees EC, Infante JR, Cohen RB, O'Neil BH, Jones S, von Mehren M, Danaee H, Lee Y, Ecsedy J, Manfredi M, Galvin K, Stringer B, Liu H, Eton O, Fingert H, Burris H. "Phase 1 study of MLN8054, a selective inhibitor of Aurora A kinase in patients with advanced solid tumors." Cancer Chemother Pharmacol. 2011, 67(4):945-54.

DeRose YS, Gligorich KM, Wang G, Georgelas A, Bowman P, Courdy SJ, Welm AL, Welm BE. "Patient-derived models of human breast cancer: protocols for in vitro and in vivo applications in tumor biology and translational medicine." Curr Protoc Pharmacol. 2013 Mar; Chapter 14:Unit14.23.

Hait, W.N. "Anticancer drug development: the grand challenges." Nat Rev Drug Discov. 2010 Apr;9(4):253-4.

Ice RJ, McLaughlin SL, Livengood RH, Culp MV, Eddy ER, Ivanov AV, Pugacheva EN. "NEDD9 depletion destabilizes Aurora A kinase and heightens the efficacy of Aurora A inhibitors: 
implications for treatment of metastatic solid tumors." Cancer Res. 2013 May 15;73(10):3168-80.

Ishikawa F, Yasukawa M, Lyons B, Yoshida S, Miyamoto T, Yoshimoto G, Watanabe T, Akashi K, Shultz LD, Harada M. "Development of functional human blood and immune systems in NOD/SCID/IL2 receptor \{gamma\} chain(null) mice." Blood. 2005 Sep 1;106(5):156573. Epub 2005 May 26.

Press JZ, Kenyon JA, Xue H, Miller MA, De Luca A, Miller DM, Huntsman DG, Gilks CB, McAlpine JN, Wang YZ. "Xenografts of primary human gynecological tumors grown under the renal capsule of NOD/SCID mice show genetic stability during serial transplantation and respond to cytotoxic chemotherapy." Gynecol Oncol. 2008 Aug;110(2):256-64.

Pugacheva, E.N. and E.A. Golemis, "The focal adhesion scaffolding protein HEF1 regulates activation of the Aurora-A and Nek2 kinases at the centrosome." Nat Cell Biol, 2005. 7(10): p. 937-46.

Nadler Y, Camp RL, Schwartz C, Rimm DL, Kluger HM, Kluger Y.Expression of Aurora A (but not Aurora B) is predictive of survival in breast cancer." Clin Cancer Res, 2008. 14(14): p. $4455-62$.

Manfredi MG, Ecsedy JA, Meetze KA, Balani SK, Burenkova O, Chen W, Galvin KM, Hoar KM, Huck JJ, LeRoy PJ, Ray ET, Sells TB, Stringer B, Stroud SG, Vos TJ, Weatherhead GS, Wysong DR, Zhang M, Bolen JB, Claiborne CF. "Antitumor activity of MLN8237, an orally active small-molecule inhibitor of Aurora A kinase." Proc Natl Acad Sci U S A, 2007. 104(10): p. 4106-11.

Motz GT, Coukos G. "Deciphering and reversing tumor immune suppression." Immunity. 2013 Jul 25;39(1):61-73.

Reyal F, Guyader C, Decraene C, Lucchesi C, Auger N, Assayag F, De Plater L, Gentien D, Poupon MF, Cottu P, De Cremoux P, Gestraud P, Vincent-Salomon A, Fontaine JJ, Roman-Roman S, Delattre O, Decaudin D, Marangoni E. "Molecular profiling of patientderived breast cancer xenografts." Breast Cancer Res. 2012 Jan 16;14(1):R11.

Siegel R, DeSantis C, Virgo K, Stein K, Mariotto A, Smith T, Cooper D, Gansler T, Lerro C, Fedewa S, Lin C, Leach C, Cannady RS, Cho H, Scoppa S, Hachey M, Kirch R, Jemal A, Ward E. "Cancer treatment and survivorship statistics, 2012." CA Cancer J Clin. 2012 Jul-Aug;62(4):220-41.

Tanaka T, Kimura M, Matsunaga K, Fukada D, Mori H, Okano Y. "Centrosomal kinase AlK1 is overexpressed in invasive ductal carcinoma of the breast." Cancer Res, 1999. 59(9): p. 2041-4.

Voskoglou-Nomikos, T., Pater, J.L. \& Seymour, L. "Clinical predictive value of the in vitro cell line, human xenograft, and mouse allograft preclinical cancer models." Clin. Cancer Res. 9, 4227-4239 (2003). 


\section{Figures:}

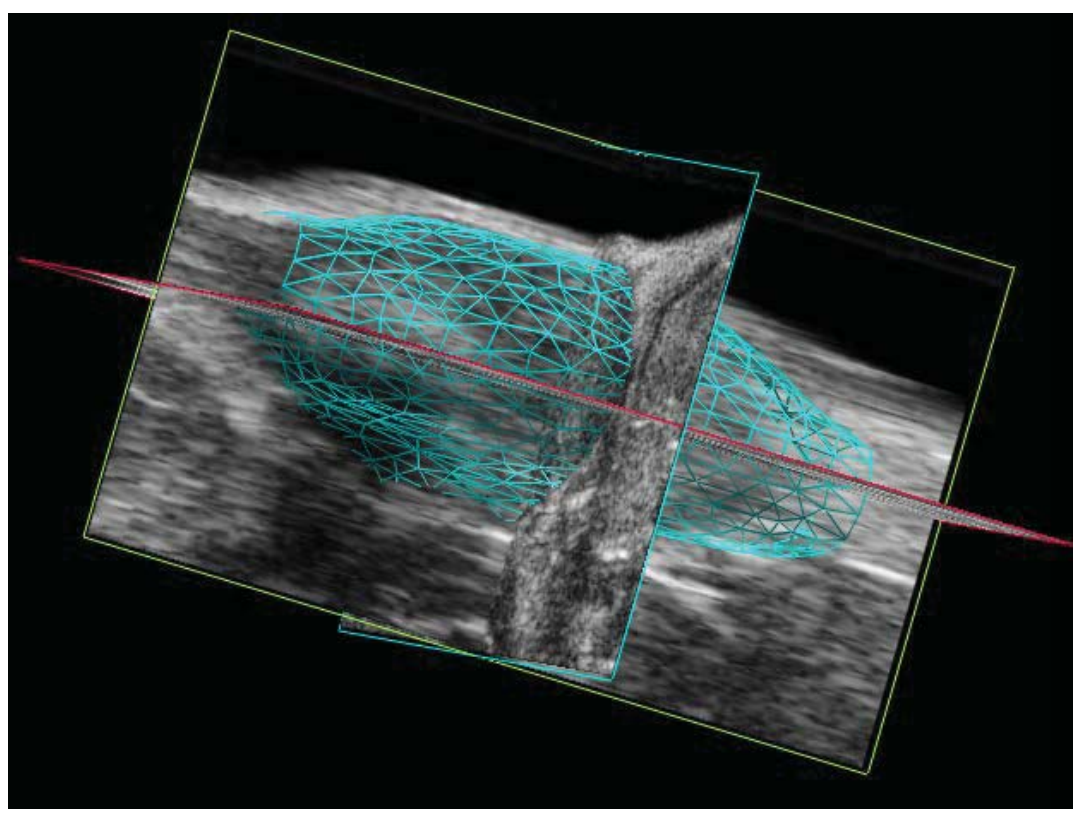

Figure 1. Successful engraftment of patient derived xenograft tumor. A Composite of VEVO Ultrasound images taken at four weeks post-surgery of PEN_003 engraftment. Tumor volume equaled $60.63 \mathrm{~mm}^{3}$. 


\begin{tabular}{|c|c|c|c|c|c|c|c|c|c|}
\hline PDX_ID & $\begin{array}{ll}\text { PDX } & \text { ID } \\
\text { (original) }\end{array}$ & $\begin{array}{l}\text { NATI } \\
\text { MAL }\end{array}$ & Diagnosis & Source & Sex & Age & Race & $\begin{array}{l}\text { Treat } \\
\text { ment }\end{array}$ & $\begin{array}{l}\text { ER/PR } \\
\text { /Her2 }\end{array}$ \\
\hline PEN_001 & M1131301A9 & MAL & IDC & Ohio St & $\mathrm{F}$ & 81 & W & & $+/-/-$ \\
\hline PEN_002 & HCl_001 & MAL & IDC & Utah & $\mathrm{F}$ & $\mathrm{N} / \mathrm{A}$ & $\mathrm{N} / \mathrm{A}$ & None & $-/-/-$ \\
\hline PEN_003 & $\mathrm{HCl}+002$ & MAL & IDC & Utah & $\mathrm{F}$ & $\mathrm{N} / \mathrm{A}$ & $\mathrm{N} / \mathrm{A}$ & None & $-/-/-$ \\
\hline PEN_004 & HCl_009 & MAL & $\begin{array}{l}\text { adeno- } \\
\text { carcinoma }\end{array}$ & Utah & $\mathrm{F}$ & $\mathrm{N} / \mathrm{A}$ & $N / A$ & Chemo & $-/-/-$ \\
\hline PEN_005 & HCl_010 & MAL & IDC & Utah & $\mathrm{F}$ & $\mathrm{N} / \mathrm{A}$ & $N / A$ & Chemo & $-/-/-$ \\
\hline PEN_006 & M1132001A1 & MAL & IDC & Ohio St & $\mathrm{F}$ & 56 & W & & $-/+/-$ \\
\hline PEN_010 & M1132321A2 & MAL & $\begin{array}{l}\text { papillary } \\
\text { carcinoma }\end{array}$ & Ohio St & $\mathrm{F}$ & 67 & W & & \\
\hline PEN_012 & ED63092T_003 & MAL & IDC & UPenn & $\mathrm{F}$ & 35 & $W$ & Chemo & \\
\hline PEN_014 & ED63407T_003 & MAL & carcinoma & UPenn & $\mathrm{F}$ & 58 & $B$ & Chemo & \\
\hline PEN_016 & ED63423T_004 & MAL & IDC & UPenn & $\mathrm{F}$ & 32 & W & None & \\
\hline PEN_018 & ED63703T_004 & MAL & IDC & UPenn & $\mathrm{F}$ & 49 & W & None & \\
\hline PEN_020 & ED62917_T003 & MAL & carcinoma & UPenn & $\mathrm{F}$ & 64 & W & None & \\
\hline PEN_021 & M1132517A2 & MAL & IDC & Ohio St & $\mathrm{F}$ & 61 & $\mathrm{~W}$ & & \\
\hline
\end{tabular}

Table 1. Patient Derived Xenograft Tumor Tissue Received.

Table abbreviations: NAT, normal adjacent to tumor; MAL, malignant; IDC, invasive ductal carcinoma; Chemo, chemotherapy; F, female 


\section{General Discussion}

Collectively, the studies executed in the course of this dissertation work present multiple novel insights pertaining to the role of NEDD9 in tumorigenesis, metastasis, and cancer treatment. In Study 1, we demonstrated that NEDD9 protects AURKA from degradation through blocking $\mathrm{CDH} 1$ binding and therefore increasing the amount of AURKA present in the tumor cell. We were also able to establish that in patient samples the levels of AURKA and NEDD9 are correlative, indicating that the phenomenon is present in patient tumors. Additionally, we discovered the major finding that the AURKA inhibitor MLN8237 (Alisertib) greatly reduced metastasis via two different complimentary methodologies and that by reducing the levels of NEDD9 we were able to sensitize the tumor cells thereby virtually eliminating all signs of metastasis. Although the precise mechanism of action is an area that will need further investigation, these results indicate a clear clinical use for AURKA inhibitors in treating metastatic disease and the use of NEDD9 as a biomarker for AURKA inhibitor susceptibility.

Furthermore, the ability of NEDD9 to increase invasion and metastasis in breast cancer through regulating TIMP2 and therefore MMP14 in Study 2 provides a novel mechanism for NEDD9 dependent invasion and migration. Although, previous studies have found NEDD9 to increase invasion and migration via increasing Src and FAK activity (Law 1996), the addition of yet another mechanism points to the fact that siRNA based therapies need to be developed to target NEDD9.

In order to investigate the effects of NEDD9 overexpression in the context of in vivo tumorigenesis we developed a novel inducible mouse line in Study 3. Through our developing of a Cre recombinase inducible NEDD9 knock-in mouse line, we were able to investigate the role of NEDD9 in mammary gland development. Illustrating that, overexpression of NEDD9 leads to an increase in mammary gland bud to branch ratio indicating enhanced development. Interestingly, mammary gland development has been shown to be a MMP dependent process, and while it will take more investigation to prove that the ability of NEDD9 to regulate MMP activity in vivo is indeed the mechanism of action, it is a promising area for research. 
Lastly, in our striving to better recapitulate human breast cancer we have developed the capability to utilize the novel method of patient derived xenografts. By creating a tissue bank of human tissue samples and cataloging tumors which engrafted we will have an inexhaustible supply of human tissue and at the same time be able to treat the tumor of a patient with therapies that are not even developed yet. More to our purpose, we will be able to test the ability of the AURKA inhibitor MLN8237 to treat metastatic breast cancers which phenocopy the metastatic sites present in patients. Additionally, we will be able to find biomarkers indicating susceptibility to AURKA inhibitors without the need of a full clinical trial. Although the influence of an immune system is a hurdle that needs to be overcome, the development of the patient derived xenograft system we will be able to more quickly weed out therapies that are ineffective in human disease, and thusly be able to progress with greater success in clinical trials.

\section{References}

Law SF, Estojak J, Wang B, Mysliwiec T, Kruh G, Golemis EA."Human enhancer of filamentation 1, a novel p130cas-like docking protein, associates with focal adhesion kinase and induces pseudohyphal growth in Saccharomyces cerevisiae." Mol Cell Biol. 1996 Jul;16(7):3327-37. 